JOURNAL OF THE

AMERICAN MATHEMATICAL SOCIETY

Volume 11, Number 1, January 1998, Pages 119-174

S 0894-0347(98)00250-1

\title{
VIRTUAL MODULI CYCLES AND GROMOV-WITTEN INVARIANTS OF ALGEBRAIC VARIETIES
}

\author{
JUN LI AND GANG TIAN
}

\section{INTRODUCTION}

The study of moduli spaces plays a fundamental role in our understanding of the geometry and topology of manifolds. One example is Donaldson theory (and more recently the Seiberg-Witten invariants), which provides a set of differential invariants of 4-manifolds [Do]. When the underlying manifolds are smooth algebraic surfaces, then they are the intersection theories on the moduli spaces of vector bundles over these surfaces $[\mathrm{Li}],[\mathrm{Mo}]$. Another example is the mathematical theory, inspired by the sigma model theory in mathematical physics ([W1], [W2]), of quantum cohomology. The quantum cohomology uses the GW-invariants, which are the intersection numbers of certain induced homology classes on the moduli spaces of rational curves in a given symplectic manifold. This is a generalization of the classical enumerative invariant which counts the number of algebraic curves with appropriate constraints in a variety. The first mathematical foundation of quantum cohomology was established by Ruan and the second named author in [RT1] for semi-positive symplectic manifolds, which include all algebraic manifolds of complex dimension less than 4, all Fano manifolds and Calabi-Yau spaces. In [RT2], general GW-invariants of higher genus are constructed to establish a mathematical theory of the sigma model theory coupled with gravity on any semi-positive symplectic manifolds (also see $[\mathrm{Ru}]$ for the special cases). There are some related works we would like to mention. In [KM], Kontsevich and Manin proposed an axiomatic approach to $\mathrm{GW}$-invariants, and in [Ko2], Kontsevich introduced the notion of stable maps to study GW-invariants. There are also works dealing with special classes of Fano varieties, such as homogeneous manifolds (cf. [BDW], [Ber], [Ci], $[\mathrm{CM}],[\mathrm{LT}])$.

Now let us discuss the new issue in intersection theory raised from studying GW-invariants, and more generally the Donaldson type invariants. The core of an intersection theory is the fundamental class. For a manifold (or a variety), the ordinary cup product with the fundamental class given by the underlying manifold provides a satisfactory intersection theory. However, for the GW-invariants, which should be an intersection theory on the moduli space of stable maps, we cannot take the fundamental class of the whole moduli space directly. This is because the relative moduli space (i.e., the family version) in general does not form a flat

Received by the editors September 25, 1996 and, in revised form, July 30, 1997.

1991 Mathematics Subject Classification. Primary 14D20.

Key words and phrases. Moduli space, intersection theory, invariant.

Both authors were supported in part by NSF grants, and the first author was also supported by an A. Sloan fellowship and Terman fellowship. 
family over the parameter space. One guiding principle of our search of a "good" intersection theory is that such a theory should be invariant under deformation of the underlying manifolds. In [Do], [RT1], [RT2], the authors employed analytic methods to construct "good" intersection theory using generic moduli spaces (they are almost always non-algebraic).

Abiding with algebraic methods, we don't have the luxury of having a "generic moduli space". Instead, we will construct directly a cycle in the moduli space, called the virtual moduli cycle, and define an intersection theory by using this cycle as the fundamental class. Such a construction commutes with Gysin maps. In this paper, we will construct such a cycle by first constructing a cone cycle inside a vector bundle, which functions as a normal cone, and then intersecting this cone cycle with the zero section of the vector bundle. To make this construction sufficiently general, we shall carry it out based on the moduli functor solely. The data we need is a choice of tangent-obstruction complex of the moduli functor, which is a global obstruction theory of the moduli problem. The virtual moduli cycle depends on the choice of such a complex, so does the virtual intersection theory defined. The so-constructed intersection theory will have the following invariance property. Given a family of moduli functors, namely, a relative moduli functor, if we assume that the tangent-obstruction complex of the relative moduli functor and that of the specialized moduli functor are compatible, then the specialization of the virtual intersection theory on the relative moduli space is the same as the virtual intersection theory of the specialized moduli space. Applying to the moduli space of stable maps from $n$-pointed nodal curves into a smooth projective variety $X$, we can define the GW-invariants of $X$ purely algebraically.

We now describe briefly the key idea to our construction. When we are working with a moduli space, usually we can compute its virtual dimension. However, the virtual dimension may not coincide with the actual dimension of the moduli space. One may view this as if the moduli space is a subspace of an "ambient" space cut out by a set of "equations" whose vanishing loci do not meet properly. Such a situation is well understood in the following setting: let

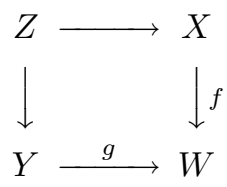

be a fiber square, where $X, Y$ and $W$ are smooth varieties and subvarieties. Then $[X] \cdot[Y]$, the intersection of the cycle $[X]$ and $[Y]$, is a cycle in $A_{*} W$ of dimension $\operatorname{dim} X+\operatorname{dim} Y-\operatorname{dim} W$. When $\operatorname{dim} Z=\operatorname{dim} X+\operatorname{dim} Y-\operatorname{dim} W$, then $[Z]=[X] \cdot[Y]$. Otherwise, $[Z]$ may not be $[X] \cdot[Y]$. The excess intersection theory tells us that we can find a cycle in $A_{*} Z$ so that it is $[X] \cdot[Y]$. We may view this cycle as the virtual cycle of $Z$ representing $[X] \cdot[Y]$. Following Fulton-MacPherson's normal cone construction, this cycle is the image of the cycle of the normal cone to $Z$ in $X$, denoted by $C_{Z / X}$, under the Gysin homomorphism $s^{*}: A_{*}\left(C_{Y / W} \times_{Y} Z\right) \rightarrow A_{*} Z$, where $s: Z \rightarrow C_{Y / W} \times_{Y} Z$ is the zero section. This theory does not apply directly to moduli schemes, since, except for some isolated cases, it is impossible to find pairs $X \rightarrow W$ and $Y \rightarrow W$ so that $X \times_{W} Y$ is the moduli space and $[X] \cdot[Y]$ so defined is the virtual moduli cycle we need.

The strategy to our approach is that rather than trying to find an embedding of the moduli space into some ambient space, we will construct a cone in a vector 
bundle directly, say $C \subset V$, over the moduli space and then define the virtual moduli cycle to be $s^{*}[C]$, where $s$ is the zero section of $V$. The pair $C \subset V$ will be constructed based on a choice of the tangent-obstruction complex of the moduli functor. (The definition of tangent-obstruction complex is given in section 1.)

Let $\mathcal{M}$ be a moduli space. We first construct its tangent-obstruction complex, which usually comes from studying obstruction theory of the moduli problem. For a large class of moduli problems, their tangent-obstruction complexes are the sheaf cohomologies of complexes of locally free sheaves

$$
\mathcal{E}^{\bullet}=\left[\mathcal{E}_{1} \longrightarrow \mathcal{E}_{2}\right]
$$

Assume that $\mathcal{M}$ belongs to this class of moduli problems. Then at each closed point $w \in \mathcal{M}$,

$$
T_{1}=\mathfrak{h}^{1}\left(\mathcal{E}^{\bullet} \otimes_{\mathcal{O}_{\mathcal{M}}} k(w)\right)
$$

is the tangent space $T_{w} \mathcal{M}$ and

$$
T_{2}=\mathfrak{h}^{2}\left(\mathcal{E}^{\bullet} \otimes_{\mathcal{O}_{\mathcal{M}}} k(w)\right)
$$

is the obstruction space to deformations of $w$ in $\mathcal{M}$. Here $\mathfrak{h}^{i}\left(\mathcal{E}^{\bullet}\right)$ is the $i$-th sheaf cohomology of the complex. There is an "intrinsic" set of defining equations of the germ of $\mathcal{M}$ at $w$, namely, the Kuranishi map

$$
f: \hat{\operatorname{Sym}} \bullet\left(T_{2}^{\vee}\right) \longrightarrow \hat{\operatorname{Sym}} \bullet\left(T_{1}^{\vee}\right):=\lim _{\longleftarrow} \oplus_{l=0}^{n} S^{l}\left(T_{1}^{\vee}\right) .
$$

Note that if we denote by $\hat{w}$ the formal completion of $\mathcal{M}$ along $w$, then [La]

$$
\hat{w} \cong \operatorname{Spec~} \hat{\operatorname{Sym}} \bullet\left(T_{1}^{\vee}\right) \otimes_{\operatorname{Sym}} \bullet\left(T_{2}^{\vee}\right)
$$

The normal cone to $\hat{w}$ in $\operatorname{Spec} \hat{\operatorname{Sym}} \bullet\left(T_{1}^{\vee}\right)$ is canonically a subcone in $\hat{w} \times_{k} T_{2}$. We denote this cone by $C_{w}$. The virtual normal cone we seek will be a cycle $[C]$ in $Z_{*} \operatorname{Vect}\left(\mathcal{E}_{2}\right)$, where $\operatorname{Vect}\left(\mathcal{E}_{2}\right)$ is the vector bundle over $\mathcal{M}$ so that its sheaf of sections is $\mathcal{E}_{2}$. Then $[C]$ is uniquely determined by the following criterion. At each $w \in \mathcal{M}$, there is a surjective vector bundle homomorphism

$$
\operatorname{Vect}\left(\mathcal{E}_{2}\right) \times_{\mathcal{M}} \hat{w} \longrightarrow \operatorname{Vect}\left(T_{2}\right) \times \hat{w},
$$

where $T_{2}=\mathfrak{h}^{2}\left(\mathcal{E}^{\bullet} \otimes_{\mathcal{O}_{\mathcal{M}}} k(w)\right)$, that extends the given homomorphism $\mathcal{E}_{2} \rightarrow$ $\mathfrak{h}^{2}\left(\mathcal{E}^{\bullet} \otimes_{\mathcal{O}_{\mathcal{M}}} k(w)\right)$ such that the restriction of $[C]$ to $\operatorname{Vect}\left(\mathcal{E}_{2}\right) \times_{\mathcal{M}} \hat{w}$ is the pull back of $C_{w}$. In short, the virtual normal cone is the result of patching these local normal cones defined by the Kuranishi maps of the moduli space. The virtual moduli cycle $[\mathcal{M}]^{\text {vir }}$ is then defined to be the image of $[C]$ in $A_{*} \mathcal{M}$ via the Gysin homomorphism

$$
s^{*}: A_{*} \operatorname{Vect}\left(\mathcal{E}_{2}\right) \rightarrow A_{*} \mathcal{M} .
$$

The GW-invariants are defined by applying this construction to the moduli spaces of stable morphisms from nodal curves to $X$.

Theorem. For any smooth projective variety $X$, and any choice of integers $n$ and $g$ and $\alpha \in A_{1} X / \sim_{\text {alg, }}$, there is a virtual moduli cycle $\left[\mathcal{M}_{\alpha, g, n}^{X}\right]^{\text {vir }} \in A_{k} X \otimes_{\mathbf{z}} \mathbf{Q}$, where $k$ is the virtual dimension of $\mathcal{M}_{\alpha, g, n}^{X}$. Using this cycle, we can define the $G W$-invariants

$$
\Psi_{\alpha, g, n}^{X}:\left(A^{*} X\right)^{\times n} \times A^{*} \mathcal{M}_{g, n} \longrightarrow A_{*} \mathcal{M}_{\alpha, g, n}^{X} \otimes_{\mathbf{z}} \mathbf{Q}
$$


in the usual way. Let $\psi_{\alpha, g, n}^{X}$ be the composite of $\Psi_{\alpha, g, n}^{X}$ with the degree homomorphism $A_{*} \mathcal{M}_{\alpha, g, n}^{X} \rightarrow \mathbf{Q}$. Then $\psi_{\alpha, g, n}^{X}$ are invariant under deformations of $X$ and satisfy all the expected properties of the $G W$-invariant, including the composition law.

The construction of the virtual moduli cycle $\left[\mathcal{M}_{\alpha, g, n}^{X}\right]^{\text {vir }}$ is the main purpose of this paper. The proof of the composition law is almost straightforward, following a similar process as in [RT1]. Since maps in $\mathcal{M}_{\alpha, g, n}^{X}$ may have non-trivial automorphisms, our classes may have rational coefficients. The approach to this problem is the usual descent argument. In the end, we obtain a cycle supported on an effective cone over $\mathcal{M}_{\alpha, g, n}^{X}$ inside a $\mathbf{Q}$-vector bundle. The virtual moduli cycle is then the image of the Gysin map as described before. The resulting class is of rational coefficients.

This construction of virtual cycles was finished in early 1995. During the AMS summer meeting held at Santa Cruz, July, 1995, the first named author reported this work. In his talk, he described the ideas of our construction of virtual moduli cycles and the definition of GW-invariants. After the talk, S. Katz kindly informed the first named author that he had studied the problem of constructing virtual moduli cycles and obtained some partial results in special cases [Kz1]. During the preparation of this paper, we learned that K. Behrend and B. Fantechi had given an alternative construction of virtual moduli cycles [BF]. Also as an application, $\mathrm{K}$. Behrend defined GW-invariants and proved the basic property of these invariants [Bh]. A similar idea can be applied to constructing symplectic invariants for general symplectic manifolds.

The layout of this paper is as follows. In section one, we introduce the notion of tangent-obstruction complexes of functors, which is a global obstruction theory of the moduli functors. We then describe the tangent-obstruction complex of the moduli functor of stable morphisms. The next two sections are devoted to the construction and investigation of the virtual normal cone of any tangent-obstruction complex. In sections 4 and 5, we will construct the GW-invariants and prove some basic properties of these invariants, including their deformation invariance and the composition laws.

The first named author thanks W. Fulton and D. Gieseker for many stimulating discussions. Part of this work was done when the second named author visited the Department of Mathematics, Stanford University, in the winter quarter of 1994. He would like to thank his colleagues there for providing a stimulating atmosphere. We thank the referee for many comments and suggestions.

\section{TANGEnT-OBStRUCtion COMPLEX}

In this section we will introduce the notion of tangent-obstruction complexes of moduli functors. Such a notion was implicit in many earlier works and should be viewed as another way of presenting deformation theory.

In this paper, we will fix an algebraically closed field $k$ of characteristic 0 and will only consider schemes over $k$.

We first define the functor of tangent spaces. Let $\mathcal{S}$ be the category of all schemes and let $\mathfrak{F}: \mathcal{S} \rightarrow$ (sets) be a (contravariant) moduli functor. Here we call $\mathfrak{F}$ a moduli functor if for any $S \in \mathcal{S}$ the object $\mathfrak{F}(S)$ is the set of isomorphism classes of flat families of objects (to be parameterized) over $S$. For our purpose, we will introduce an associated functor, called the pre-moduli functor of $\mathfrak{F}$ and denoted by $\mathfrak{F}^{\text {pre }}$. For 
any $S \in \mathcal{S}, \mathfrak{F}^{\text {pre }}(S)$ is the set of all flat families of objects (to be parameterized) over $S$. Note that we do not take isomorphism classes in this case. Following the convention, for $\xi_{1}, \xi_{2} \in \mathfrak{F}(S)$ we will denote by $\xi_{1} \cong \xi_{2}$ the case when $\xi_{1}$ and $\xi_{2}$ are isomorphic and denote by $\sim$ the equivalence relation induced by $\cong$. Hence $\mathfrak{F}(S)=\mathfrak{F}^{\text {pre }}(S) / \sim$. In this paper, for $S \in \mathcal{S}$ we let $\mathfrak{M}^{\circ} \mathfrak{d}_{S}$ be the category of sheaves of $\mathcal{O}_{S}$-modules. For $\mathcal{N} \in \mathfrak{M o d}_{S}$, we denote by $S_{N}$ the trivial extension of $S$ by the sheaf $\mathcal{N},{ }^{1}$ and denote by $\pi_{\mathcal{N}}: \mathfrak{F}^{\text {pre }}\left(S_{N}\right) \rightarrow \mathfrak{F}^{\text {pre }}(S)$ the restriction morphism induced by the obvious inclusions $S \subset S_{N}$. Given $\eta_{0} \in \mathfrak{F}^{\text {pre }}(S)$ and $\zeta_{1}, \zeta_{2} \in \pi_{\mathcal{N}}^{-1}\left(\eta_{0}\right)$, we say $\zeta_{1} \cong \eta_{0} \zeta_{2}$ if there is an isomorphism $\rho: \zeta_{1} \cong \zeta_{2}$ so that its restriction $\pi_{\mathcal{N}}(\rho): \eta_{0} \cong \eta_{0}$ is the identity isomorphism. Now we define the functor of tangent spaces. For any affine $S \in \mathcal{S}$ and $\eta_{0} \in \mathfrak{F}^{\text {pre }}(S)$, we let $\mathcal{T} \mathfrak{F}\left(\eta_{0}\right): \mathfrak{M o d}_{S} \rightarrow(\text { sets })^{0}$ be the functor that assigns $\mathcal{N}$ to the set $\pi_{\mathcal{N}}^{-1}\left(\eta_{0}\right)$ modulo the equivalence relation induced by the isomorphism $\cong \eta_{0}$. In short, $\mathcal{T} \mathfrak{F}\left(\eta_{0}\right)$ consists of all isomorphism classes of $\tilde{\eta} \in \mathfrak{F}^{\text {pre }}\left(S_{N}\right)$ whose restriction to $S$ is $\eta_{0}$. In case $\eta_{0} \cong \eta_{0}^{\prime}$, then there is a canonical isomorphism of sets $\mathcal{T} \mathfrak{F}\left(\eta_{0}\right)$ and $\mathcal{T} \mathfrak{F}\left(\eta_{0}^{\prime}\right)$. This way, for $\eta \in \mathfrak{F}(S)$ we obtain an isomorphism class of functors $\mathcal{F} \mathfrak{F}\left(\eta_{0}\right): \mathfrak{M o d}_{S} \rightarrow(\text { sets })^{0}$. It is clear that if $\rho: S_{1} \rightarrow S_{2}$ is a morphism between affine schemes and if $\mathcal{N}_{1} \in \mathfrak{M}^{\prime} \mathfrak{d}_{S_{1}}$ and $\mathcal{N}_{2} \in \mathfrak{M}^{\prime} d_{S_{2}}$ are two sheaves with $\mathcal{O}_{S_{1}}$-homomorphism $\mathcal{O}_{S_{1}} \otimes_{\mathcal{O}_{S_{2}}} \mathcal{N}_{2} \rightarrow \mathcal{N}_{1}$, then for any $\eta_{2} \in \mathfrak{F}(S)$ with $\eta_{1}=\mathfrak{F}(\rho)\left(\eta_{2}\right)$ the induced object in $\mathfrak{F}\left(S_{1}\right)$, there is a canonical morphism

$$
\mathcal{T} \mathfrak{F}\left(\eta_{2}\right)\left(\mathcal{N}_{2}\right) \longrightarrow \mathcal{T} \mathfrak{F}\left(\eta_{1}\right)\left(\mathcal{N}_{1}\right),
$$

satisfying the base change property. Note that when $\mathfrak{F}$ is represented by a scheme $Y$ and $\eta \in \mathfrak{F}(S)$ is represented by a morphism $f: S \rightarrow Y$, then

$$
\mathcal{T} \mathfrak{F}(\eta)(\mathcal{N})=\Gamma\left(\mathcal{H o m}_{S}\left(f^{*} \Omega_{Y}, \mathcal{N}\right)\right) .
$$

In this case, $\mathcal{T} \mathfrak{F}(\eta)$ is a functor $\mathfrak{M o d}_{S} \rightarrow \mathfrak{M o d}_{S}$.

Assumption. In this paper, we will only consider the moduli functor $\mathfrak{F}$ such that $\mathcal{T} \mathfrak{F}$ is induced by a sheaf-valued functor over fibered category of modules over schemes over $\mathfrak{F}$. Namely, for any affine $S \in \mathfrak{M o d}_{S}, \eta \in \mathfrak{F}(S)$ and $\mathcal{N} \in \mathfrak{M o d}_{S}$ the set $\mathcal{T} \mathfrak{F}(\eta)(\mathcal{N})$ is canonically isomorphic to the set of all sections of a sheaf of $\mathcal{O}_{S}$-modules, denoted by $\mathcal{T}^{1} \mathfrak{F}(\eta)(\mathcal{N})$, and the arrows (above) in the base change property are induced by sheaf homomorphisms

$$
\mathcal{T}^{1} \mathfrak{F}\left(\eta_{2}\right)\left(\mathcal{N}_{2}\right) \otimes_{\mathcal{O}_{S_{2}}} \mathcal{O}_{S_{1}} \longrightarrow \mathcal{T}^{1} \mathfrak{F}\left(\eta_{1}\right)\left(\mathcal{N}_{1}\right)
$$

In the following, we will call $\mathcal{T}^{1} \mathfrak{F}$ the functor of tangent spaces. We remark that we have not exhausted the literature to see how restrictive this assumption is. Nevertheless, the moduli functors that will be discussed in this paper all satisfy this condition.

Next, we recall the definition of an obstruction theory. An obstruction theory to deformations of $p \in \mathfrak{F}(\operatorname{Spec} k)$ with values in a vector space $O$ is an assignment as follows. Given a pair $(\eta \in \mathfrak{F}(\operatorname{Spec} B / I), I \subset B)$, where $B$ is an Artin ring with the residue field $k, I \subset B$ is an ideal annihilated by the maximal ideal of $B$ and $\eta \otimes_{B / I} k=p$, the obstruction theory assigns a natural obstruction class $\mathrm{ob}(\eta, B / I, B) \in O$ whose vanishing is the necessary and sufficient condition for $\eta$ to be extendible to $\tilde{\eta} \in \mathfrak{F}(\operatorname{Spec} B)$. We now introduce its relative analogue.

\footnotetext{
${ }^{1}$ By this we mean $S_{N}=\operatorname{Spec}\left(\Gamma\left(\mathcal{O}_{S}\right) * \Gamma(\mathcal{N})\right)$, where $\Gamma\left(\mathcal{O}_{S}\right) * \Gamma(\mathcal{N})$ is the trivial ring extension of $\Gamma\left(\mathcal{O}_{S}\right)$ by $\Gamma(\mathcal{N})$. Note that there is an inclusion $S \rightarrow S_{N}$ and projection $S_{N} \rightarrow S$ so that $S \rightarrow S_{N} \rightarrow S$ is the identity. (See [Ma, p. 191].)
} 
Definition 1.1. Let $\mathcal{K}=\left\{\mathcal{K}_{\eta}\right\}$ be a collection of sheaves of $\mathcal{O}_{S}$-modules $\mathcal{K}_{\eta}$ indexed by $\eta$ for $S \in \mathcal{S}$ and $\eta \in \mathfrak{F}(S)$. We say that $\mathcal{K}$ is a sheaf over $\mathfrak{F}$ if for any morphism $f: T \rightarrow S$ of schemes, there is an isomorphism $f^{*} \mathcal{K}_{\eta} \cong \mathcal{K}_{f^{*} \eta}$ canonical under base change.

Definition 1.2. An obstruction theory of the moduli functor $\mathfrak{F}$ with values in a sheaf ob• over $\mathfrak{F}$ consists of the following data: let $S$ be any affine scheme, let $S \rightarrow Y_{0} \rightarrow Y$ be schemes and embedding morphisms over $S$. Namely, $Y_{0} \rightarrow Y$ is an embedding and $i: S \rightarrow Y_{0}$ is a section of $Y_{0} \rightarrow S$. Let $\mathfrak{m}$ be the ideal sheaf of $S \subset Y$ and let $\mathcal{I} \subset \mathcal{O}_{Y}$ be the ideal sheaf of $Y_{0} \subset Y$. Assume that $\mathcal{I} \cdot \mathfrak{m}=0$. Then for any $\eta \in \mathfrak{F}\left(Y_{0}\right)$, there is an obstruction class

$$
\mathrm{ob}\left(\eta, Y_{0}, Y\right) \in \Gamma_{S}\left(\mathrm{ob}_{\eta_{0}} \otimes_{\mathcal{O}_{S}} \mathcal{I}\right)
$$

where $\eta_{0}=i^{*}(\eta) \in \mathfrak{F}(S)$, whose vanishing is the necessary and sufficient condition for $\eta$ to be extendible to $\tilde{\eta} \in \mathfrak{F}(Y)$. We call ob $\left(\eta, Y_{0}, Y\right)$ the obstruction class to extending $\eta$ to $Y$. The obstruction class is canonical under base change: let $\sigma: S^{\prime} \rightarrow S$ be another morphism, $Y^{\prime}$ a scheme over $S^{\prime}$ with a section $i^{\prime}$ and $f: Y^{\prime} \rightarrow Y$ a morphism such that

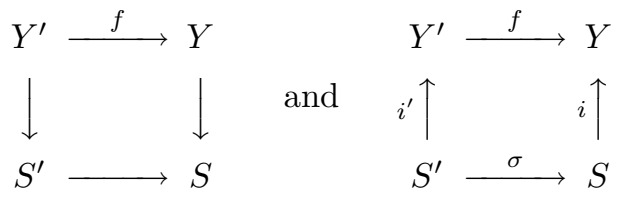

are commutative. Let $Y_{0}^{\prime}=Y_{0} \times_{Y} Y^{\prime}$ and let $\eta^{\prime} \in \mathfrak{F}\left(S^{\prime}\right)$ be the pull back of $\eta$. Let $g: \sigma^{*}\left(\mathrm{ob}_{\eta_{0}} \otimes_{\mathcal{O}_{S}} \mathcal{I}_{Y_{0} \subset Y}\right) \rightarrow \mathrm{ob}_{\eta_{0}^{\prime}} \otimes_{\mathcal{O}_{S}} \mathcal{I}_{Y_{0}^{\prime} \subset Y^{\prime}}$ be the obvious homomorphism. Then

$$
\mathrm{ob}\left(\eta^{\prime}, Y_{0}^{\prime}, Y^{\prime}\right)=g\left(\mathrm{ob}\left(\eta, Y_{0}, Y\right)\right) \text {. }
$$

Example. Let $X \subset \mathbf{A}^{n}$ be a subscheme defined by the ideal $I=\left(f_{1}, \ldots, f_{m}\right)$. Let $\mathfrak{F}_{X}$ be the functor $\operatorname{Mor}(-, X)$ and let $\mathcal{C} \bullet$ be the complex $\left[\mathcal{O}_{X}\left(T \mathbf{A}^{n}\right) \stackrel{\sigma}{\longrightarrow} \mathcal{O}_{X}^{\oplus m}\right]$ where $\sigma=\left(d f_{1}, \ldots, d f_{m}\right)$. Then for any affine $S$, morphism $\eta: S \rightarrow X$ and sheaf $\mathcal{N} \in \mathfrak{M o d}_{S}, \mathcal{T}^{1} \mathfrak{F}_{X}(\eta)(\mathcal{N})$ is the first sheaf cohomology of $\eta^{*} \mathcal{C}^{\bullet} \otimes_{\mathcal{O}_{S}} \mathcal{N}$. The defining sections $f_{1}, \ldots, f_{m}$ define an obstruction theory of the functor $\mathfrak{F}_{X}$ with values in $\operatorname{coker}(\sigma)$ (see section 2 for an explicit description).

Example ([Al]). This example concerns the moduli of stable sheaves $\mathcal{E}$ on a smooth algebraic surface $X$ of a fixed Poincaré polynomial $\chi$. Here we implicitly fix an ample divisor on $X$. We denote the corresponding moduli functor by $\mathfrak{F}_{\chi}$. For any affine $S \in \mathcal{S}$ and $\eta \in \mathfrak{F}_{\chi}(S)$ representing the sheaf $\mathcal{E}$ of $\mathcal{O}_{X \times S}$-modules, then

$$
\mathcal{T}^{1} \mathfrak{F}_{\chi}(\eta)(\mathcal{N})=\mathcal{E} x t_{S \times X / S}^{1}\left(\mathcal{E}, \mathcal{E} \otimes \pi_{S}^{*} \mathcal{N}\right)^{0},
$$

where the superscript means the traceless part of the extension sheaf. The canonical obstruction theory of $\mathfrak{F}_{\chi}$ takes values in the sheaf $\mathcal{E} x t_{S \times X / S}^{2}(\mathcal{E}, \mathcal{E})^{0}$.

Let ob. be the sheaf in Definition 1.2. For simplicity, we will use the convention $\mathcal{T}^{2} \mathfrak{F}(\eta)(\mathcal{N})=\mathrm{ob}_{\eta} \otimes_{\mathcal{O}_{S}} \mathcal{N}$ and $\mathcal{T}^{\bullet} \mathfrak{F}=\left[\mathcal{T}^{1} \mathfrak{F} \rightarrow \mathcal{T}^{2} \mathfrak{F}\right]$, where the arrow is the zero homomorphism. By the assumption of this section, $\mathcal{T}^{1} \mathfrak{F}(\eta)(\mathcal{N})$ is a two-term complex of sheaves of $\mathcal{O}_{S}$-modules connected by the zero arrow.

Definition 1.3. Let $\mathfrak{F}$ be as before. A tangent-obstruction complex of $\mathfrak{F}$ is a complex $\mathcal{T}^{\bullet} \mathfrak{F}=\left[\mathcal{T}^{1} \mathfrak{F} \rightarrow \mathcal{T}^{2} \mathfrak{F}\right]$, where the arrow is the zero arrow, such that $\mathcal{T}^{1} \mathfrak{F}$ is the functor of the tangent spaces of $\mathfrak{F}$ and that there is an obstruction theory of $\mathfrak{F}$ taking values in $\mathcal{T}^{2} \mathfrak{F}$. The tangent-obstruction complex $\mathcal{T}^{\bullet} \mathfrak{F}$ is said to be a perfect 
tangent-obstruction complex if for any affine $S$ and $\eta \in \mathfrak{F}(S)$, there is an affine covering $\left\{S_{\alpha}\right\}$ of $S$ such that there are two-term complexes of locally free sheaves $\mathcal{E}_{\alpha}^{\bullet}$ such that for any $\mathcal{N} \in \mathfrak{M o d}_{S_{\alpha}}, \mathcal{T}^{i} \mathfrak{F}\left(\eta_{\alpha}\right)(\mathcal{N})$ is the $i$-th sheaf cohomology of $\mathcal{E}_{\eta_{\alpha}}^{\bullet} \otimes_{\mathcal{O}_{S_{\alpha}}} \mathcal{N}$, where $\eta_{\alpha} \in \mathfrak{F}\left(S_{\alpha}\right)$ is the induced object of $\eta$ via $S_{\alpha} \rightarrow S$. In case the complex $\mathcal{E}^{\bullet}$ is explicitly given, we will write $\mathcal{T} \bullet \mathfrak{F}=\mathfrak{h}^{\bullet}\left(\mathcal{E}^{\bullet}\right)$.

We emphasize that the obstruction theory is part of the data making up the tangent-obstruction complex. This notion is a convenient way to group the data of tangent spaces and obstruction theory. It will be clear later that when the tangentobstruction complex is perfect, then the classical construction of Kuranishi maps can be adopted to construct the relative Kuranishi families, which is the heart of the construction of virtual moduli cycles.

Remark. The assumption that $\mathfrak{F}$ admits a perfect tangent-obstruction complex is a strong requirement. For instance, moduli functors of stable vector bundles over threefolds other than Calabi-Yau manifolds may not have perfect tangentobstruction complexes.

In this paper, our main interest is in the moduli spaces of stable morphisms from marked curves to smooth projective varieties. Let $X$ be a fixed smooth projective variety. We first recall the notion of stable morphisms introduced by Kontsevich [Ko1]. An $n$-pointed nodal curve is a nodal curve $C$ and $n$ ordered marked points $D \subset C$ away from the singular locus of $C$ (we will use $D$ to denote the $n$-ordered marked points on $C$ in this paper). A morphism $f: D \subset C \rightarrow X$ is said to be stable if $D \subset C$ is an $n$-pointed connected nodal curve and $f: C \rightarrow X$ is a morphism such that

$$
\operatorname{Hom}_{C}\left(\Omega_{C}(D), \mathcal{O}_{C}\right) \rightarrow \operatorname{Hom}_{C}\left(f^{*} \Omega_{X}, \mathcal{O}_{C}\right)
$$

is surjective, where $f^{*} \Omega_{X} \rightarrow \Omega_{C}(D)$ is induced by $f^{*} \Omega_{X} \rightarrow \Omega_{C}$. We will call $f$ stable relative to $D$ or simply stable if the marked points $D \subset C$ are understood.

From now on, we fix a class $\alpha \in A_{1} X / \sim_{\text {alg }}$ and two integers $n$ and $g$. We let $\mathfrak{F}_{\alpha, g, n}^{X}: \mathcal{S} \rightarrow(\text { sets })^{0}$ be the functor that assigns any $S \in \mathcal{S}$ to the set of all isomorphism classes of flat families over $S$ of stable morphisms

$$
f: D \subset \mathcal{X} \longrightarrow X
$$

from $n$-pointed connected nodal curves $D \subset \mathcal{X}$ of arithmetic genus $g$ to $X$ such that $f$ sends the fundamental classes of closed fibers of $\mathcal{X}$ over $S$ to $\alpha$. Since $X$ is a smooth projective variety, by the work of $[\mathrm{Al}], \mathfrak{F}_{\alpha, g, n}^{X}$ is coarsely represented by a projective scheme. We denote this scheme by $\mathcal{M}_{\alpha, g, n}^{X}$. It is also known that $\mathfrak{F}_{\alpha, g, n}^{X}$ is represented by a Deligne-Mumford stack [FP].

In the following, we will determine the natural tangent-obstruction complex of $\mathfrak{F}_{\alpha, g, n}^{X}$. We fix an affine scheme $S$ and a sheaf $\mathcal{N} \in \mathfrak{M}^{\prime} \mathfrak{d}_{S}$. Let $\xi \in \mathfrak{F}_{\alpha, g, n}^{X}(S)$ be represented by $f: \mathcal{X} \rightarrow X$ with marked sections $D \subset \mathcal{X}$. Let $\mathcal{X}^{N}$ be a flat family of nodal curves over $S_{N}$, where $S_{N}$ is the trivial extension of $S$ by $\mathcal{N}$, that extends the family $\mathcal{X}$. Then we have a commutative diagram of exact sequences

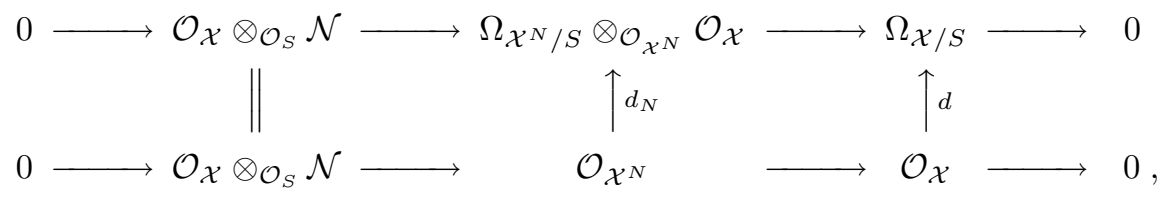


where $d$ and $d_{N}$ are the differentials. The upper sequence is exact because $\mathcal{X}^{N}$ is a family of nodal curves flat over $S_{N}$, following Theorem 25.2 in [Ma]. Conversely, given any exact sequence of sheaves of $\mathcal{O}_{\mathcal{X}}$-modules

$$
0 \longrightarrow \mathcal{O}_{\mathcal{X}} \otimes_{\mathcal{O}_{S}} \mathcal{N} \longrightarrow \mathcal{C} \longrightarrow \Omega_{\mathcal{X} / S} \longrightarrow 0,
$$

we obtain a pull-back from the $S$-homomorphism $d: \mathcal{O}_{\mathcal{X}} \rightarrow \Omega_{\mathcal{X} / S}$ :

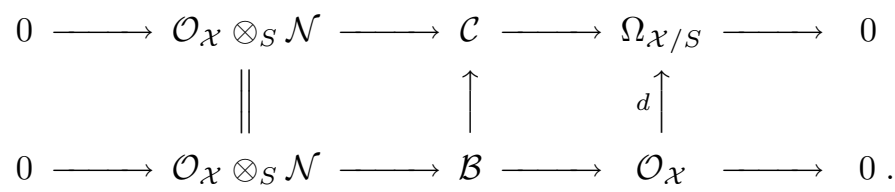

One checks that there is a canonical way to give $\mathcal{B}$ a sheaf structure of $\mathcal{O}_{S_{N}}$-algebras, which is flat over $S_{N}$ automatically. Thus we obtain a flat family $\mathcal{X}^{N}$ over $S_{N}$. One checks also that this correspondence is one-to-one and onto. This is the one-to-one correspondence between the space of flat extensions of $\mathcal{X} \rightarrow S$ to $\mathcal{X}^{N} \rightarrow S_{N}$ and the module

$$
\operatorname{Ext}_{\mathcal{X} / S}^{1}\left(\Omega_{\mathcal{X} / S}, \mathcal{O}_{\mathcal{X}} \otimes_{\mathcal{O}_{S}} \mathcal{N}\right)
$$

Next we investigate when such an extension $\mathcal{X}^{N}$ admits a morphism $f^{N}: \mathcal{X}^{N} \rightarrow$ $X$ extending $f: \mathcal{X} \rightarrow X$. We claim that such an $f^{N}$ comes from the existence of an $\mathcal{O}_{\mathcal{X}}$-linear lifting $f^{*} \Omega_{X} \rightarrow \Omega_{\mathcal{X}^{N} / S} \otimes_{\mathcal{O}_{\mathcal{X}^{N}}} \mathcal{O}_{\mathcal{X}}$ of the obvious $f^{*} \Omega_{X} \rightarrow \Omega_{\mathcal{X} / S}$. Indeed, given $f^{N}$ restricting to $f$, we certainly have such a lifting from

$$
\left(f^{N}\right)^{*} \Omega_{X} \longrightarrow \Omega_{\mathcal{X}^{N} / S} \text { and }\left(f^{N}\right)^{*} \Omega_{X} \otimes_{\mathcal{O}_{\mathcal{X}^{N}}} \mathcal{O}_{\mathcal{X}}=f^{*} \Omega_{X}
$$

Conversely, given any diagram

$$
\begin{aligned}
& f^{*} \Omega_{X}=f^{*} \Omega_{X} \\
& 0 \longrightarrow \mathcal{O}_{\mathcal{X}} \otimes_{S} \mathcal{N} \longrightarrow \mathcal{B} \longrightarrow \Omega_{\mathcal{X} / S} \longrightarrow 0,
\end{aligned}
$$

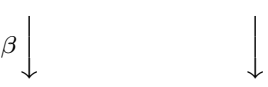

we first obtain a flat extension $\mathcal{X}^{N}$ of $\mathcal{X}$ and an isomorphism $\mathcal{B} \cong \Omega_{\mathcal{X}^{N} / S} \otimes_{\mathcal{O}_{\mathcal{X}^{N}}} \mathcal{O}_{\mathcal{X}}$ based on the bottom exact sequence. Observing that

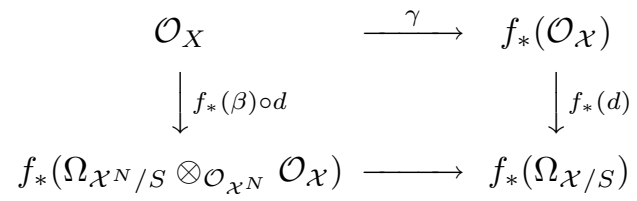

is commutative, we can factor $f_{*}(\beta) \circ d$ and $\gamma$ through $\mathcal{O}_{X} \rightarrow f_{*}\left(\mathcal{O}_{\mathcal{X}^{N}}\right)$, because

$$
\begin{aligned}
& f_{*}\left(\mathcal{O}_{\mathcal{X}^{N}}\right) \longrightarrow f_{*}\left(\mathcal{O}_{\mathcal{X}}\right) \\
& \downarrow f_{*}\left(d_{N}\right) \quad \downarrow f_{*}(d) \\
& f_{*}\left(\Omega_{\mathcal{X}^{N} / S} \otimes_{\mathcal{O}_{\mathcal{X}}} \mathcal{O}_{\mathcal{X}}\right) \longrightarrow f_{*}\left(\Omega_{\mathcal{X} / S}\right)
\end{aligned}
$$

is a pull-back diagram. One checks directly that $\mathcal{O}_{X} \rightarrow f_{*}\left(\mathcal{O}_{\mathcal{X}^{N}}\right)$ is a homomorphism of sheaves of $S$-algebras. Therefore, it defines a morphism $f^{N}: \mathcal{X}^{N} \rightarrow X$ that is an extension of $f: \mathcal{X} \rightarrow X$.

In conclusion, we have shown that for any affine $S \in \mathcal{S}$ and $\xi \in \mathfrak{F}_{\alpha, g, 0}^{X}(S)$ that corresponds to the family $f: \mathcal{X} \rightarrow X$, the tangent $\mathcal{T} \mathfrak{F}_{\alpha, g, 0}^{X}$ at $\xi$ takes $\mathcal{N} \in \mathfrak{M o d}_{S}$ 
to the set of all commutative diagrams of $\mathcal{O}_{\mathcal{X}}$-modules (1.1). This set is naturally the first extension module

$$
\operatorname{Ext}_{\mathcal{X}}^{1}\left(\left[f^{*} \Omega_{X} \rightarrow \Omega_{\mathcal{X} / S}\right], \mathcal{O}_{\mathcal{X}} \otimes_{\mathcal{O}_{S}} \mathcal{N}\right)
$$

where $\left[f^{*} \Omega_{X} \rightarrow \Omega_{\mathcal{X} / S}\right]$ is a complex indexed at -1 and 0 . We should point out that in $[\mathrm{Ra}], \mathrm{Z}$. Ran has identified the deformation space and the obstruction space of this moduli problem to the above diagram and has expressed them in terms of extension modules over non-commutative rings. He actually treated more general cases. Based on $[\mathrm{Ra}]$ and the above reasoning, we only need to check that the set of diagrams above is canonically isomorphic to the above extension module. This can be checked by using hypercohomology of a double complex based on a covering of $\mathcal{X}_{S}$ and a locally free resolution of $\Omega_{\mathcal{X}_{S}}$, similar to the example in [GH]. We leave the details to the readers.

Now we give the tangent of the functor $\mathfrak{F}_{\alpha, g, n}^{X}$.

Proposition 1.4. Let $S$ be any affine scheme and let $\xi \in \mathfrak{F}_{\alpha, g, n}^{X}(S)$ be represented by $f: \mathcal{X} \rightarrow X$ with marked points $D \subset \mathcal{X}$. Then for any $\mathcal{N} \in \mathfrak{M}^{\circ} d_{S}$,

$$
\mathcal{T}^{1} \mathfrak{F}_{\alpha, g, n}^{X}(\xi)(\mathcal{N})=\mathcal{E} x t_{\mathcal{X} / S}^{1}\left(\left[f^{*} \Omega_{X} \rightarrow \Omega_{\mathcal{X} / S}(D)\right], \mathcal{O}_{\mathcal{X}} \otimes_{\mathcal{O}_{S}} \mathcal{N}\right)
$$

Proof. We will sketch the proof of one direction and leave the other to the readers. Given any section in the above sheaf, we can associate to it a diagram

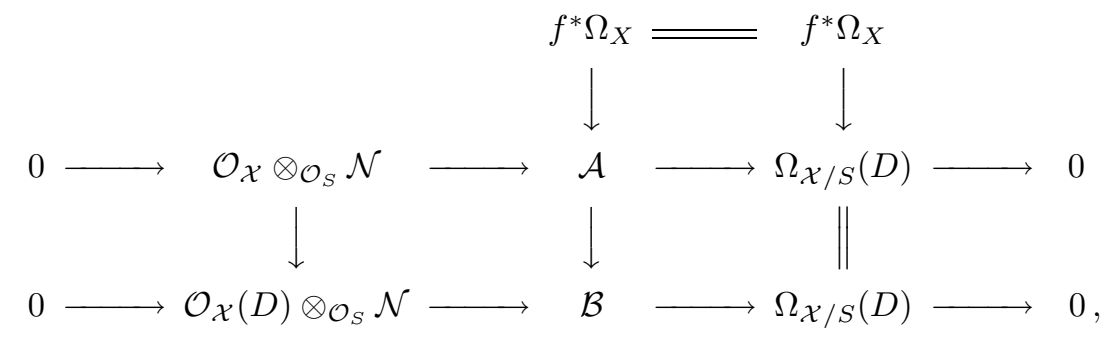

where the lower left square is the push-forward of sheaves of $\mathcal{O}_{\mathcal{X}}$-modules. The last line (tensored by $\mathcal{O}_{\mathcal{X}}(-D)$ ) defines an extension $\mathcal{X}^{N}$. Since $f^{*} \Omega_{X} \rightarrow \Omega_{\mathcal{X} / S}(D)$ factors through $\Omega_{\mathcal{X} / S} \subset \Omega_{\mathcal{X} / S}(D), f^{*} \Omega_{X} \rightarrow \mathcal{B}$ factors through $\mathcal{B}(-D) \subset \mathcal{B}$, and thus defines a morphism $f^{N}: \mathcal{X}^{N} \rightarrow X$. The immersion $D^{N} \rightarrow \mathcal{X}^{N}$ extending $D \rightarrow \mathcal{X}$ is determined by the data $\operatorname{coker}\{\mathcal{A} \rightarrow \mathcal{B}\}$. In this way, we have constructed an extension

$$
f^{N}: D^{N} \subset \mathcal{X}^{N} \longrightarrow X \text { of } f: D \subset \mathcal{X} \longrightarrow X .
$$

It is routine to check that this correspondence is one-to-one and onto, and satisfies the required base change property. This proves the proposition.

We now describe the standard choice of the obstruction theory of $\mathfrak{F}_{\alpha, g, n}^{X}$.

Proposition 1.5. For any $S \in \mathcal{S}$ and $\eta \in \mathfrak{F}_{\alpha, g, n}^{X}(S)$ corresponding to the family $f: \mathcal{X} \rightarrow X$ over $S$ with the marked sections $D$, we define $\mathrm{ob}_{\eta}$ to be the sheaf

$$
\mathcal{E x t}_{\mathcal{X} / S}^{2}\left(\left[f^{*} \Omega_{X} \rightarrow \Omega_{\mathcal{X} / S}(D)\right], \mathcal{O}_{\mathcal{X}}\right) .
$$

We let $\mathrm{ob}$. be the collection $\left\{\mathrm{ob}_{\eta}\right\}$ indexed by $\eta \in \mathfrak{F}(S)$ for $S \in \mathcal{S}$. Then ob• forms a sheaf over $\mathfrak{F}_{\alpha, g, n}^{X}$. Furthermore, there is an obstruction theory of $\mathfrak{F}_{\alpha, g, n}^{X}$ taking values in the sheaf ob. 
Proof. It is clear that $\left\{\mathrm{ob}_{\eta}\right\}$ is a sheaf over $\mathfrak{F}_{\alpha, g, n}^{X}$. Now we describe the obstruction theory taking values in this sheaf. Let $S$ be any affine scheme and let $S \rightarrow Y_{0} \rightarrow Y$ be a tuple of $S$-schemes described in Definition 1.2 (namely, $S \rightarrow Y_{0} \rightarrow Y$ are embeddings and the ideal sheaf of $Y_{0} \subset Y$ is annihilated by the ideal sheaf of $S \subset Y)$. Let $\eta \in \mathfrak{F}_{\alpha, g, n}^{X}\left(Y_{0}\right)$ be any object corresponding to a family $f_{0}: \mathcal{X}_{0} \rightarrow X$ with marked points $D_{0} \subset \mathcal{X}_{0}$ understood. We let $\overline{\mathcal{X}}=\mathcal{X}_{0} \times_{Y_{0}} S, \bar{D}=D_{0} \times_{Y_{0}} S$ and $\bar{f}=\left.f_{0}\right|_{\overline{\mathcal{X}}}$. We let $\bar{\pi}: \overline{\mathcal{X}} \rightarrow S$ be the projection. Since $\mathcal{I}:=\mathcal{I}_{Y_{0} \subset Y}$ is annihilated by the ideal sheaf $\mathcal{I}_{S \subset Y}, \mathcal{I}$ is a sheaf of $\mathcal{O}_{S}$-modules. Clearly, $D_{0} \subset \mathcal{X}_{0}$ can be extended to a family over $Y$, say $D \subset \mathcal{X}$. Since $\mathcal{X} \rightarrow Y$ is a flat family of nodal curves, we obtain an exact sequence

$$
0 \longrightarrow \mathcal{I}_{\mathcal{X}_{0} \subset \mathcal{X}} \longrightarrow \Omega_{\mathcal{X} / S} \otimes_{\mathcal{O}_{\mathcal{X}}} \mathcal{O}_{\mathcal{X}_{0}} \longrightarrow \Omega_{\mathcal{X}_{0} / S} \longrightarrow 0 .
$$

Let $\tau(\mathcal{X}) \in \operatorname{Hom}\left(f_{0}^{*} \Omega_{X}, \Omega_{\mathcal{X}_{0} / S}\right)$ be the obvious homomorphism and let

$$
\bar{\tau}(\mathcal{X}) \in \operatorname{Ext}_{\overline{\mathcal{X}}_{0}}^{1}\left(\bar{f}^{*} \Omega_{X}, \bar{\pi}^{*} \mathcal{I}\right)
$$

be the image of $\tau(\mathcal{X})$ under the connecting homomorphism

$$
\operatorname{Hom}\left(f_{0}^{*} \Omega_{X}, \Omega_{\mathcal{X}_{0} / S}\right) \stackrel{\delta}{\longrightarrow} \operatorname{Ext}_{\mathcal{X}_{0}}^{1}\left(f_{0}^{*} \Omega_{X}, \mathcal{I}_{\mathcal{X}_{0} \subset \mathcal{X}}\right)=\operatorname{Ext}_{\overline{\mathcal{X}}}^{1}\left(\bar{f}^{*} \Omega_{X}, \bar{\pi}^{*} \mathcal{I}\right) .
$$

Here the second identity holds because $f_{0}^{*} \Omega_{X}$ is locally free. It follows that $\bar{\tau}(\mathcal{X})=0$ if and only if $f_{0}^{*} \Omega_{X} \rightarrow \Omega_{\mathcal{X}_{0} / S}$ lifts to $f_{0}^{*} \Omega_{X} \rightarrow \Omega_{\mathcal{X} / S} \otimes_{\mathcal{O}_{\mathcal{X}}} \mathcal{O}_{\mathcal{X}_{0}}$. This can be shown by similar arguments in studying $\mathcal{T} \mathfrak{F}_{\alpha, g, n}^{X}(\eta)$ that it is the necessary and sufficient condition for $f_{0}: \mathcal{X}_{0} \rightarrow X$ to be extendible to $f: \mathcal{X} \rightarrow X$. We let

$$
\operatorname{ob}\left(\eta, Y_{0}, Y\right) \in \operatorname{Ext}_{\mathcal{X}}^{2}\left(\left[\bar{f}^{*} \Omega_{X} \rightarrow \Omega_{\overline{\mathcal{X}} / S}(\bar{D})\right], \bar{\pi}^{*} \mathcal{I}\right)
$$

be the image of $\bar{\tau}(\mathcal{X})$ under the obvious homomorphism

$$
\begin{aligned}
\operatorname{Ext}_{\overline{\mathcal{X}}}^{1}\left(\bar{f}^{*} \Omega_{X}, \bar{\pi}^{*} \mathcal{I}\right) \rightarrow \operatorname{Ext}_{\mathcal{X}}^{2}\left(\left[\bar{f}^{*} \Omega_{X} \rightarrow \Omega_{\overline{\mathcal{X}} / S}\right], \bar{\pi}^{*} \mathcal{I}\right) & \cong \operatorname{Ext}_{\overline{\mathcal{X}}}^{2}\left(\left[\bar{f}^{*} \Omega_{X} \rightarrow \Omega_{\overline{\mathcal{X}} / S}(\bar{D})\right], \bar{\pi}^{*} \mathcal{I}\right) .
\end{aligned}
$$

To complete the proof, we need to check that the definition of ob $\left(\eta_{0}, Y_{0}, Y\right)$ is independent of the choice of extension $D \subset \mathcal{X}, \mathrm{ob}\left(\eta_{0}, Y_{0}, Y\right)$ has the required base change property and is the obstruction to extending $f$ to $Y$. Since the choice of the marked points of the nodal curve is irrelevant to extending $f_{0}$ to $f$ and since the definition of $\mathrm{ob}\left(\eta_{0}, Y_{0}, Y\right)$ is independent of the choice of the marked points, to study the obstruction problem, it suffices to look at the situation where $D=\emptyset$. We will assume this in the rest of this section. We now check that $\operatorname{ob}\left(\eta_{0}, Y_{0}, Y\right)$ is independent of the choice of the extension $\mathcal{X}$. Indeed, let $\mathcal{X}^{\prime}$ be another extension over $Y$. Then by the deformation theory of nodal curves, there is an extension class $v \in \operatorname{Ext}_{\overline{\mathcal{X}}}^{1}\left(\Omega_{\overline{\mathcal{X}} / S}, \bar{\pi}^{*} \mathcal{I}\right)$ defining the exact sequence

$$
0 \longrightarrow \bar{\pi}^{*} \mathcal{I} \longrightarrow \mathcal{A} \longrightarrow \Omega_{\overline{\mathcal{X}} / S} \longrightarrow 0,
$$

of which the following holds. Let

$$
0 \longrightarrow\left(\bar{\pi}^{*} \mathcal{I}\right)^{\oplus 2} \longrightarrow \Omega_{\mathcal{X} / S} \otimes_{\mathcal{O}_{\mathcal{X}}} \mathcal{O}_{\mathcal{X}_{0}} \oplus \mathcal{A}^{\prime} \longrightarrow \Omega_{\mathcal{X}_{0} / S} \longrightarrow 0
$$


be the exact sequence induced by (1.2), (1.3) and $\Omega_{\mathcal{X}_{0} / S} \rightarrow \Omega_{\overline{\mathcal{X}} / S}$. Then the bottom exact sequence in

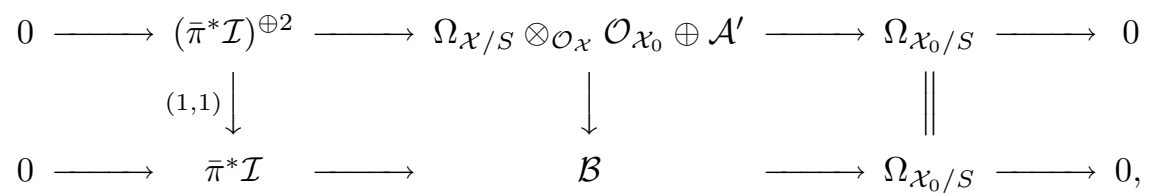

where the left square is the push forward of sheaves, is isomorphic to the exact sequence

$$
0 \longrightarrow \bar{\pi}^{*} \mathcal{I} \longrightarrow \Omega_{\mathcal{X}^{\prime} / S} \otimes_{\mathcal{O}_{\mathcal{X}^{\prime}}} \mathcal{O}_{\mathcal{X}_{0}} \longrightarrow \Omega_{\mathcal{X}_{0} / S} \longrightarrow 0 .
$$

Therefore, $\bar{\tau}\left(\mathcal{X}^{\prime}\right)=\bar{\tau}(\mathcal{X})+\delta_{0}(v)$, where

$$
\delta_{0}: \operatorname{Hom}\left(\bar{f}^{*} \Omega_{X}, \Omega_{\overline{\mathcal{X}} / S}\right) \rightarrow \operatorname{Ext}^{1}\left(\bar{f}^{*} \Omega_{X}, \bar{\pi}^{*} \mathcal{I}\right)
$$

is the obvious connecting homomorphism, and hence the images of $\bar{\tau}(\mathcal{X})$ and $\bar{\tau}\left(\mathcal{X}^{\prime}\right)$ in $\operatorname{Ext}_{\overline{\mathcal{X}}}^{2}\left(\left[\bar{f}^{*} \Omega_{X} \rightarrow \Omega_{\overline{\mathcal{X}} / S}\right], \bar{\pi}^{*} \mathcal{I}\right)$ coincide. This proves that $\mathrm{ob}\left(\eta_{0}, Y_{0}, Y\right)$ is welldefined. For the same reason, the class $\mathrm{ob}(\cdot, \cdot, \cdot)$ satisfies the required base change property.

It remains to show that $\mathrm{ob}\left(\eta_{0}, Y_{0}, Y\right)$ is the obstruction to extending $\eta$ to $Y$. Obviously, if $\eta_{0}$ can be extended to $\eta \in \mathfrak{F}_{\alpha, g, n}^{X}(Y)$, say $f: \mathcal{X} \rightarrow X$, we can take $\mathcal{X}$ to be the extension of $\mathcal{X}_{0}$ and then $\bar{\tau}(\mathcal{X})=0$ by construction. Hence ob $\left(\eta_{0}, Y_{0}, Y\right)=0$. Now assume ob $\left(\eta_{0}, Y_{0}, Y\right)=0$. Because of the exact sequence

$$
\operatorname{Ext}_{\overline{\mathcal{X}}}^{1}\left(\Omega_{\overline{\mathcal{X}} / S}, \bar{\pi}^{*} \mathcal{I}\right) \stackrel{\beta}{\longrightarrow} \operatorname{Ext}_{\overline{\mathcal{X}}}^{1}\left(\bar{f}^{*} \Omega_{X}, \bar{\pi}^{*} \mathcal{I}\right) \longrightarrow \operatorname{Ext}_{\overline{\mathcal{X}}}^{2}\left(\left[\bar{f}^{*} \Omega_{X} \rightarrow \Omega_{\overline{\mathcal{X}} / S}\right], \bar{\pi}^{*} \mathcal{I}\right) \longrightarrow 0,
$$

$\bar{\tau}(\mathcal{X})$ is $\beta(-v)$ for some $v \in \operatorname{Ext}_{\overline{\mathcal{X}}}^{1}\left(\Omega_{\overline{\mathcal{X}} / S}, \bar{\pi}^{*} \mathcal{I}\right)$. It follows from the deformation theory of nodal curves that we can find an extension $\mathcal{X}^{\prime}$ over $Y$ (of $\mathcal{X}_{0}$ ) such that the diagrams of exact sequence (1.3)-(1.6) hold. Hence $\bar{\tau}\left(\mathcal{X}^{\prime}\right)=\bar{\tau}(\mathcal{X})+\beta(v)=0$, which implies that $f$ extends to $f^{\prime}: \mathcal{X}^{\prime} \rightarrow X$. This proves that ob $\left(\eta_{0}, Y_{0}, T\right)$ is the obstruction class to extending $\eta_{0}$ to $Y$.

In section 4 , we will show that $\mathcal{T} \mathfrak{F}_{\alpha, g, n}^{X}$ is a perfect tangent-obstruction complex of $\mathfrak{F}_{\alpha, g, n}^{X}$.

\section{Relative Kuranishi families}

In this section, we will construct the relative Kuranishi families of a perfect tangent-obstruction complex. We will show that any two such families are equivalent under an explicit transformation. This will be used to construct virtual normal cones and cycles of moduli spaces in the next section.

We begin with the notion of relative tangent-obstruction complex and the observation on how defining an equation induces the relative tangent-obstruction complex. In this section, we assume that $S$ is an affine scheme and $Z$ is a formal $S$-scheme with a section $i: S \rightarrow Z$ so that as sets $\operatorname{Supp}(Z)=\operatorname{Supp}(i(S))$. We further assume that there is a finite rank locally free sheaf $\mathcal{F}$ of $\mathcal{O}_{S}$-modules such that $Z$ is embedded in $\operatorname{Spec} \hat{\operatorname{Sym}} \bullet \mathcal{F})$. (Recall $\hat{\operatorname{Sym}} \bullet(\mathcal{F})=\lim _{\leftarrow} \oplus_{l=0}^{n} S^{n}(\mathcal{F})$.)

Definition 2.1. Let $Z / S$ be as before. A perfect relative tangent-obstruction complex is a two-term complex $\left[\mathcal{E}_{1} \stackrel{\sigma}{\rightarrow} \mathcal{E}_{2}\right]$ of locally free sheaves of $\mathcal{O}_{S}$-modules for which the following hold.

(1) The cokernel of $\mathcal{E}_{2}^{\vee} \stackrel{\sigma^{\vee}}{\rightarrow} \mathcal{E}_{1}^{\vee}$ is isomorphic to $\Omega_{Z / S} \otimes_{\mathcal{O}_{Z}} \mathcal{O}_{S}$. 
(2) Let $O=\operatorname{coker} \sigma$. Then there is a relative obstruction theory to extending $S$-morphisms to $Z$ with values in $O$.

Here, an obstruction theory to extending $S$-morphisms to $Z$ is an assignment that, to each tuple of $S$-schemes $S \rightarrow Y_{0} \rightarrow Y$ described in Definition 1.2 and any $S$-morphism $\varphi_{0}: Y_{0} \rightarrow Z$, assigns a canonical obstruction class

$$
\mathrm{ob}\left(\varphi_{0}, Y_{0}, Y\right) \in \Gamma_{S}\left(O \otimes_{\mathcal{O}_{S}} \mathcal{I}_{Y_{0} \subset Y}\right)
$$

to extending $\varphi_{0}$ to $Y \rightarrow Z$.

In this paper, for a scheme $W$, we often need to consider the formal completion of $W \times W$ along its diagonal. We denote this completion by $\hat{W}$. We will view $\hat{W}$ as a $W$-scheme where $\pi: \hat{W} \rightarrow W$ is induced by the first projection of $W \times W$. We will denote by $p_{W}: \hat{W} \rightarrow W$ the projection induced by the second projection of $W \times W$. Note that there is a canonical section $W \rightarrow \hat{W}$ of $\pi: \hat{W} \rightarrow W$ induced by the diagonal embedding $W \rightarrow W \times W$.

Lemma 2.2. Let $W$ be a quasi-projective scheme. Assume that $W$ admits a perfect tangent-obstruction complex $\mathcal{T}_{W}^{\bullet}=\mathfrak{h}^{\bullet}\left(\mathcal{E}^{\bullet}\right)$. Let $S \subset W$ be a locally closed subscheme. Then $\mathcal{T}_{W}^{\bullet}$ canonically induces a perfect relative tangent-obstruction complex of $\hat{W} \times_{W} S / S$, denoted by $\mathcal{T}_{\hat{W} \times_{W} S / S}$.

Proof. Since $\hat{W}$ is the formal completion of $W \times W$ along its diagonal,

$$
\Omega_{\hat{W} \times{ }_{W} S / S} \otimes_{\mathcal{O}_{\hat{W} \times W} S} \mathcal{O}_{S} \cong \Omega_{W} \otimes_{\mathcal{O}_{W}} \mathcal{O}_{S} .
$$

Now let $S \rightarrow Y_{0} \rightarrow Y$ be a tuple of $S$-schemes as before. Assume that $\varphi_{0}: Y_{0} \rightarrow$ $\hat{W} \times{ }_{W} S$ is an $S$-morphism. Then $p_{W} \circ \varphi_{0}$ is a morphism from $Y_{0}$ to $W$. Clearly, $\varphi_{0}$ extends to an $S$-morphism $\varphi: Y \rightarrow \hat{W} \times_{W} S$ if and only if $p_{W} \circ \varphi_{0}$ extends to $Y \rightarrow W$, which is possible if and only if the obstruction

$$
\mathrm{ob}\left(p_{W} \circ \varphi_{0}, Y_{0}, Y\right) \in \Gamma_{S}\left(\mathfrak{h}^{2}\left(\mathcal{E}^{\bullet} \otimes_{\mathcal{O}_{W}} \mathcal{O}_{S}\right) \otimes_{\mathcal{O}_{S}} \mathcal{I}_{Y_{0} \subset Y}\right)
$$

vanishes. Hence $\mathrm{ob}\left(p_{W} \circ \varphi_{0}, Y_{0}, Y\right)$ is the obstruction to extending $\varphi_{0}$ to $Y$.

To relate a Kuranishi family to an obstruction theory, we need to investigate how defining equations induce a perfect relative tangent-obstruction complex. Before we proceed, let us introduce the convention that will be used throughout this section. In this section, $S$ will always be an affine scheme. Let $\mathcal{E}_{1}$ and $\mathcal{E}_{2}$ be two locally free sheaves of $\mathcal{O}_{S}$-modules. We will assume throughout this section that $\Gamma\left(\mathcal{E}_{i}\right)$ are free $\Gamma\left(\mathcal{O}_{S}\right)$-modules. We will denote by $A$ the ring $\Gamma\left(\mathcal{O}_{S}\right)$ and by $E_{i}$ the free $A$ module $\Gamma\left(\mathcal{E}_{i}\right)$. Given an $A$-module $N$, we will denote by $\hat{\operatorname{Sym}} \bullet(N)$ the inverse limit $\lim _{\leftarrow} \oplus_{l=0}^{n} S^{l}(N)$ of the direct sum of the symmetric products of $N$. In this section, we will always use $M$ to denote $\hat{\operatorname{Sym}} \bullet\left(E_{1}^{\vee}\right)$. We denote by $M_{1} \subset M$ the ideal generated by $E_{1}^{\vee} \subset M$ and denote by $M_{k}$ the ideal $M_{1}^{k}$. For any $A$-homomorphism $F: E_{2}^{\vee} \rightarrow M$, sometimes denoted $F \in M \otimes_{A} E_{2}$, we will use $(F)$ to denote the ideal of $M$ generated by the components of $F$. We now fix an $F: E_{2}^{\vee} \rightarrow M$. We assume $(F) \subset M_{1}$. Let $\sigma: E_{1} \rightarrow E_{2}$ be the dual of $E_{2}^{\vee} \rightarrow M_{1} / M_{2} \equiv E_{1}^{\vee}$ which is induced by $F$. We let $O=\operatorname{coker}(\sigma)$.

We now describe how $F$ induces a relative tangent-obstruction complex to deformations of $S$-morphisms to $Z$. Let $S \rightarrow Y_{0} \rightarrow Y$ be a tuple of $S$-schemes as before and let $\varphi_{0}: M /(F) \rightarrow \Gamma\left(\mathcal{O}_{Y_{0}}\right)$ be an $A$-homomorphism. Let $g: M \rightarrow \Gamma\left(\mathcal{O}_{Y}\right)$ 
be a lift of $M \rightarrow M /(F) \rightarrow \Gamma\left(\mathcal{O}_{Y_{0}}\right)$. Clearly, $E_{2}^{\vee} \stackrel{F}{\rightarrow} M \stackrel{g}{\rightarrow} \Gamma\left(\mathcal{O}_{Y}\right)$ factors through $o: E_{2}^{\vee} \rightarrow \Gamma\left(\mathcal{I}_{Y_{0} \subset Y}\right)$. Let

$$
\operatorname{ob}\left(\varphi_{0}, Y_{0}, Y\right) \in O \otimes_{A} \Gamma\left(\mathcal{I}_{Y_{0} \subset Y}\right)
$$

be the image of $o$ under the obvious $E_{2} \otimes_{A} \Gamma\left(\mathcal{I}_{Y_{0} \subset Y}\right) \rightarrow O \otimes_{A} \Gamma\left(\mathcal{I}_{Y_{0} \subset Y}\right)$. We claim that this is the obstruction to extending $Y_{0} \rightarrow Z$ to $Y \rightarrow Z$. Assume ob $\left(\varphi_{0}, Y_{0}, Y\right)=$ 0 . Then $o$ lifts to an $h: E_{1}^{\vee} \rightarrow \Gamma\left(\mathcal{I}_{Y_{0} \subset Y}\right)$. Let $\hat{h}: M \rightarrow \Gamma\left(\mathcal{O}_{Y}\right)$ be the induced homomorphism. It follows that $g-\hat{h}: M \rightarrow \Gamma\left(\mathcal{O}_{Y}\right)$ factors through $M /(F) \rightarrow$ $\Gamma\left(\mathcal{O}_{Y}\right)$. Thus $\varphi_{0}$ extends. The other direction is clear. We leave it to the readers to check that such an assignment of obstruction class is canonical under base change.

Definition 2.3. Let $Z / S$ be as before. Assume that $\mathcal{T}_{Z / S}^{\bullet}=\mathfrak{h}^{\bullet}\left(\mathcal{E}^{\bullet}\right)$ is a perfect relative tangent-obstruction complex of $Z / S$. A relative Kuranishi family of $\mathcal{T}_{Z / S}^{\bullet}=$ $\mathfrak{h}^{\bullet}\left(\mathcal{E}^{\bullet}\right)$ is a pair $(F, \Phi)$, where

$$
F: E_{2}^{\vee} \longrightarrow M \quad \text { and } \quad \Phi: \operatorname{Spec}_{A} M /(F) \longrightarrow Z,
$$

for which the following hold.

(1) $\Phi$ is an $S$-isomorphism.

(2) The complex $\mathcal{E}^{\bullet}$ is identical to the sheafification of the complex $E_{1} \stackrel{\sigma}{\longrightarrow} E_{2}$ induced by $F$.

(3) The induced relative tangent-obstruction complex (from $F$ ) is identical to the relative tangent-obstruction complex $\mathcal{T}_{Z / S}^{\bullet}=\mathfrak{h}^{\bullet}\left(\mathcal{E}^{\bullet}\right)$.

If the choice of the complex $\mathcal{T}_{Z / S}^{\bullet}=\mathfrak{h}^{\bullet}\left(\mathcal{E}^{\bullet}\right)$ is understood from the context, we will simply call $(F, \Phi)$ a Kuranishi family and call $F$ a Kuranishi map.

The relative Kuranishi families of $\mathcal{T}_{Z / S}^{\bullet}=\mathfrak{h}^{\bullet}\left(\mathcal{E}^{\bullet}\right)$, if they exist, are not unique. Let $(\xi, \eta) \in \operatorname{Aut}_{A}(M) \times \operatorname{Hom}_{A}\left(E_{2}, M \otimes_{A} E_{2}\right)$ be a pair such that

$$
\xi \equiv 1_{M} \quad \bmod M_{2} \quad \text { and } \quad \eta \equiv 1_{E_{2}} \quad \bmod M_{1}
$$

We will show momentarily that if $(F, \Phi)$ is a relative Kuranishi family, then the pair $\left(F^{\prime}, \Phi^{\prime}\right)$ defined by

$$
F^{\prime}=\left(\left(1_{M} \otimes \eta\right) \circ\left(\xi \otimes 1_{E_{2}}\right)\right)(F) \quad \text { and } \quad \Phi^{\prime}=\Phi \circ \bar{\xi}
$$

is also a relative Kuranishi family. Here, $\xi \otimes 1_{E_{2}}$ and $1_{M} \otimes \eta$ are maps from $M \otimes_{A} E_{2}$ to $M \otimes_{A} E_{2}$ and $\bar{\xi}$ is the induced morphism

$$
\operatorname{Spec}_{A} M /\left(F^{\prime}\right) \stackrel{\bar{\xi}}{\longrightarrow} \operatorname{Spec}_{A} M /(\eta(F))=\operatorname{Spec}_{A} M /(F) .
$$

We will denote the pair $\left(F^{\prime}, \Phi^{\prime}\right)$ above by $(\xi, \eta)(F, \Phi)$. We will call those $(\xi, \eta)$ satisfying (2.1) transformations. Given two transformations $(\xi, \eta)$ and $\left(\xi^{\prime}, \eta^{\prime}\right)$, we define

$$
(\xi, \eta) \cdot\left(\xi^{\prime}, \eta^{\prime}\right)=\left(\xi \circ \xi^{\prime},\left(1_{M} \otimes \eta\right) \circ\left(\xi \otimes 1_{E_{2}}\right) \circ\left(1_{M} \otimes \eta^{\prime}\right)\right) .
$$

It follows that

$$
\left((\xi, \eta) \cdot\left(\xi^{\prime}, \eta^{\prime}\right)\right)(F, \Phi)=(\xi, \eta)\left(\left(\xi^{\prime}, \eta^{\prime}\right)(F, \Phi)\right)
$$

Let $\mathcal{K}$ be the set of all relative Kuranishi families and let $\mathcal{H}$ be the set of all transformations. It follows that $\mathcal{H}$ is a group acting on $\mathcal{K}$.

Proposition 2.4. Let $Z / S$ be as before and let $\mathcal{T}_{Z / S}^{\bullet}=\mathfrak{h}^{\bullet}\left(\mathcal{E}^{\bullet}\right)$ be its perfect relative tangent-obstruction complex. Then the set $\mathcal{K}$ of all relative Kuranishi families is non-empty and the group $\mathcal{H}$ acts transitively on $\mathcal{K}$. 
We let $F_{1}: E_{2}^{\vee} \rightarrow M$ be the map $E_{2}^{\vee} \rightarrow E_{1}^{\vee} \subset M$ induced by $\sigma: \mathcal{E}_{1} \rightarrow \mathcal{E}_{2}$ in the complex $\mathcal{E}^{\bullet}$. As before, we denote by $\left(F_{1}\right)$ the ideal in $M$ generated by the components of $F_{1}$. Let $J_{1}=\left(F_{1}\right)+M_{2}$. Since $\operatorname{coker}\left\{\sigma^{\vee}\right\} \cong \Omega_{Z / S}$, there is a canonical $S$-morphism

$$
\Phi_{1}: \operatorname{Spec}_{A} M / J_{1} \longrightarrow Z
$$

such that the isomorphism between $\Omega_{\left(\operatorname{Spec} M / J_{1}\right) / S} \otimes_{\mathcal{O}_{\text {Spec } M / J_{1}}} \mathcal{O}_{S}$ and $\Omega_{Z / S} \otimes_{\mathcal{O}_{Z}} \mathcal{O}_{S}$ induced by $\Phi_{1}$ is the identity map.

The existence part of Proposition 2.4 follows from the following Lemma. Recall $O=\operatorname{coker}\left\{E_{1} \rightarrow E_{2}\right\}$.

Lemma 2.5. Let the notation be as before. Then there are sequences $F_{k} \in M \otimes_{A} E_{2}$ and $\Phi_{k}$ :Spec $M /\left(\left(F_{k}\right)+M_{k}\right) \rightarrow Z$, where $k=1, \cdots$, for which the following hold.

(1) $F_{1} \in M \otimes_{A} E_{2}$ and $\Phi_{1}: \operatorname{Spec}_{A} M / J_{1} \rightarrow Z$ are given as before.

(2) $F_{k}-F_{k-1} \in M_{k} \otimes E_{2}$.

(3) Let $J_{k}=\left(F_{k}\right)+M_{k+1}$. Then the image of $F_{k}$ in $\left(J_{k-1} /\left(M_{k+1}+J_{k-1} \cdot M_{1}\right)\right) \otimes_{A}$ $O$ (using quotient $E_{2} \rightarrow O$ ) is the obstruction class

$$
o_{k}=\mathrm{ob}\left(\Phi_{k-1}, M / J_{k-1}, M /\left(M_{k+1}+J_{k-1} \cdot M_{1}\right)\right)
$$

to extending $\Phi_{k-1}$ to $\operatorname{Spec} M /\left(M_{k+1}+J_{k-1} \cdot M_{1}\right) \rightarrow Z$.

Proof. We prove the lemma by induction. Assume that we have constructed a sequence $F_{1}, \ldots, F_{k-1}$ satisfying the property of the lemma. We let

$$
I_{k-1}=M_{k+1}+J_{k-1} \cdot M_{1} \subset M .
$$

Note that $M / J_{k-1}$ is a quotient ring of $M / I_{k-1}$ and its kernel $J_{k-1} / I_{k-1}$ is annihilated by the ideal $M_{1}$. We let $f_{k-1}$ be the residue class of $F_{k-1}$ in $\left(J_{k-1} /\left(M_{k}+I_{k-1}\right)\right) \otimes_{A} E_{2}$. We claim that the sequence

$$
\left(J_{k-1} / I_{k-1}\right) \otimes_{A} E_{2} \stackrel{\left(h_{1}, h_{2}\right)}{\longrightarrow}\left(J_{k-1} /\left(M_{k}+I_{k-1}\right)\right) \otimes_{A} E_{2} \oplus\left(J_{k-1} / I_{k-1}\right) \otimes_{A} O
$$

$$
\longrightarrow\left(J_{k-1} /\left(\mathcal{M}_{k}+I_{k-1}\right)\right) \otimes_{A} O \longrightarrow 0
$$

is exact. Indeed, since $h_{2}$ is surjective and has kernel $\left(J_{k-1} / I_{k-1}\right) \otimes_{A} \operatorname{Im}\left\{E_{1} \rightarrow E_{2}\right\}$, the cokernel of $\left(h_{1}, h_{2}\right)$ is the cokernel of

$$
\left(J_{k-1} / I_{k-1}\right) \otimes_{A} \operatorname{Im}\left\{E_{1} \rightarrow E_{2}\right\} \longrightarrow\left(J_{k-1} /\left(M_{k}+I_{k-1}\right)\right) \otimes_{A} E_{2},
$$

which is the last non-zero term in (2.2). Now we consider $\left(f_{k-1}, o_{k}\right)$ in the middle group of the above exact sequence. By the induction hypothesis and the base change property of obstruction class, the images of $f_{k-1}$ and $o_{k}$ in $\left(J_{k-1} /\left(M_{k}+I_{k-1}\right)\right) \otimes_{A} O$ are the obstruction class

$$
\mathrm{ob}\left(\Phi_{k-1}, M / J_{k-1}, M /\left(M_{k}+I_{k-1}\right)\right)
$$

hence they coincide. It follows that there is an $\bar{f}_{k} \in\left(J_{k-1} / I_{k-1}\right) \otimes_{A} E_{2}$ such that its image under $\left(h_{1}, h_{2}\right)$ is $\left(f_{k-1}, o_{k}\right)$. Now we choose $F_{k}$. We first select an $F_{k}^{\prime} \in J_{k-1} \otimes_{A} E_{2}$ so that its residue class is $\bar{f}_{k}$. Since $\bar{f}_{k} \equiv f_{k-1} \bmod M_{k}$, which by definition is the residue of $F_{k-1}$ in $\left(M /\left(M_{k}+I_{k-1}\right)\right) \otimes_{A} E_{2}$, it follows that

$$
F_{k}^{\prime}-F_{k-1} \in M_{k}+J_{k-1} \cdot M_{1} \text {. }
$$

Therefore, we can find an $F_{k}$ so that $F_{k}-F_{k-1} \in M_{k}$ and $F_{k}-F_{k}^{\prime} \in J_{k-1} \cdot I_{k-1}$.

Let $J_{k}=\left(F_{k}\right)+M_{k+1}$. It remains to show that $\Phi_{k-1}$ extends to an $S$-morphism

$$
\Phi_{k}: \operatorname{Spec}_{A} M / J_{k} \longrightarrow Z .
$$


Because of the obstruction theory, it suffices to show that under $h_{2}$ the residue class of $F_{k}$ in $\left(J_{k-1} / I_{k-1}\right) \otimes_{A} E_{2}$ is mapped to the obstruction class $o_{k}$, because then the obstruction class

$$
\mathrm{ob}\left(\Phi_{k-1}, M / J_{k-1}, M / J_{k}\right)
$$

will be the image of $F_{k}$ in $\left(J_{k-1} / J_{k}\right) \otimes_{A} O$, which will be zero. But this is exactly the condition imposed on $F_{k}$ in our selection. This proves that $\Phi_{k-1}$ lifts to $\Phi_{k}$ as desired. Finally, it follows from $F_{k+1}-F_{k} \in M_{k}$ that $\lim F_{k}=F \in M \otimes_{A} E_{2}$ exists and $F-F_{k} \in M_{k}$. Also, since $\Phi_{k+1}$ is an extension of $\Phi_{k}$, the $\operatorname{limit} \lim \Phi_{k}=\Phi$ : $\operatorname{Spec}_{A} M /(F) \rightarrow Z$ is an $S$-morphism. This proves the lemma.

To complete the existence part of Proposition 2.4, it remains to show that $\Phi$ is an isomorphism and the induced perfect tangent-obstruction complex from $(F, \Phi)$ is identical to $\mathcal{T}_{Z / S}^{\bullet}=\mathfrak{h}^{\bullet}\left(\mathcal{E}^{\bullet}\right)$. We now show that $\Phi$ is an isomorphism. By our technical assumption, $Z$ embeds in $\operatorname{Spec} \hat{\operatorname{Sym}} \bullet(H)$ for some finitely generated free $A$-module $H$. Without loss of generality, we can assume that $\operatorname{rank} H=\operatorname{rank} E_{1}$. Let $N=\operatorname{Spec} \hat{\operatorname{Sym}} \bullet(H)$, let $N_{1} \subset N$ be the ideal generated by $H \subset N$ and let $N_{k}=N_{1}^{k}$. We let $K \subset N$ be the ideal of $Z \subset \operatorname{Spec} N$. Let $\Phi^{*}: N / K \rightarrow M /(F)$ be the ring homomorphism induced by $\Phi$. Because $\Phi$ induces an isomorphism between $\Omega_{M /(F)} \otimes_{M /(F)} A$ and $\Omega_{N / K} \otimes_{N / K} A, \Phi^{*}$ induces an isomorphism $N /\left(K+N_{2}\right) \cong$ $M /\left((F)+M_{2}\right)$. It follows that we can find an $A$-isomorphism $\phi: N \rightarrow M$ such that $\phi(K) \subset(F)$ and $\phi / K: N / K \rightarrow M /(F)$ is $\Phi^{*}$. We now show that $\phi(K)=(F)$. Let $k$ be the least integer so that $\phi(K)+M_{k+1} \neq(F)+M_{k+1}$. Clearly, $k$ must be at least 2 . Let $J=\phi(K)+M_{k+1}$. Since $J+M_{k}=(F)+M_{k},\left((F)+M_{k}\right) / J$ is annihilated by $M_{1}$. Now let $o$ be the obstruction class to extending $\Gamma\left(\mathcal{O}_{Z}\right)=N / K \longrightarrow M /\left((F)+M_{k}\right)$ to $\Gamma\left(\mathcal{O}_{Z}\right) \rightarrow M / J$. Because such an extension does exist, we have $o=0$. On the other hand, since $J \subset(F) \cdot M_{1}+M_{k+1}$, by the definition of $F$ the obstruction $o$ is the residue of $F$ in $\left(\left((F)+M_{k}\right) / J\right) \otimes_{A} O$. Hence

$$
F \in\left((F)+M_{k}\right) \otimes_{A} \operatorname{Im}\left\{E_{1} \rightarrow E_{2}\right\}+J \subset\left((F) \cdot M_{1}+M_{k+1}\right)+J=J .
$$

This implies that $(F)+M_{k+1}=J$, contradicting our assumption that $J \neq(F)+$ $M_{k+1}$. This proves that $\phi(K)+M_{k}=(F)+M_{k}$ for all $k$, and hence $\Phi$ is an isomorphism. The proof that the tangent-obstruction complex of $(F, \Phi)$ is $\mathcal{T}_{Z / S} \dot{ }_{/ S}=$ $\mathfrak{h}^{\bullet}\left(\mathcal{E}^{\bullet}\right)$ is straightforward, and will be omitted. The existence part of Proposition 2.4 is proved.

Now we study the group action on $\mathcal{K}$. We first check that if $T=(\xi, \eta) \in \mathcal{H}$ and $(F, \Phi) \in \mathcal{K}$, then $T(F, \Phi) \in \mathcal{K}$. Let $\left(F^{\prime}, \Phi^{\prime}\right)=T(F, \Phi)$. Because of the base change property of the obstruction class, it suffices to show that the sequence $\left(F_{k}^{\prime}, \Phi_{k}^{\prime}\right):=\left(F^{\prime}, \Phi^{\prime}\right)$ satisfies the three properties listed in Lemma 2.5. Indeed, in case $T=\left(\xi, 1_{E_{2}}\right)$, properties 1,2 and 3 in Lemma 2.5 are obviously satisfied. Property 3 also holds because $\xi: M \rightarrow M$ induces an isomorphism between $(F)+M_{k}$ and $\left(F^{\prime}\right)+M_{k}$ for all $k$. This shows that $\left(\xi, 1_{E_{2}}\right)(F, \Phi) \in \mathcal{K}$. Now we consider $\left(1_{M}, \eta\right) \in \mathcal{H}$. Let $F^{\prime}=\eta(F)$. Then since $(F)=\left(F^{\prime}\right) \subset M$, properties 1 to 3 in Lemma 2.5 hold for $\left(F^{\prime}, \Phi^{\prime}\right)$ as well. This proves that $(\xi, \eta)=(1, \eta) \cdot(\xi, 1)$ acts on $\mathcal{K}$.

Lemma 2.6. $\mathcal{H}$ acts transitively on $\mathcal{K}$.

Proof. Let $(F, \Phi)$ and $(G, \Psi)$ be any two elements in $\mathcal{K}$. By definition, we know that $(F, \Phi) \equiv(G, \Psi) \bmod M_{2}$. Now assume that there is a $k \geq 2$ so that $(F, \Phi) \equiv(G, \Psi)$ 
$\bmod M_{k}$. We will show that there is a transformation $(\xi, \eta) \in \mathcal{H}$ satisfying $\xi \equiv 1_{M}$ $\bmod M_{k}$ such that $(\xi, \eta)(F, \Phi) \equiv(G, \Psi) \bmod M_{k+1}$.

Let $J_{k-1}=(F)+M_{k}$, which is $(G)+M_{k}$ by the assumption. Let $I_{k-1}=M_{k+1}+$ $J_{k-1} \cdot M_{1}$ and let $f_{k}$ and $g_{k}$ be the residue classes of $F$ and $G$ in $\left(J_{k-1} / I_{k-1}\right) \otimes_{A} E_{2}$ respectively. Let

$$
\beta:\left(J_{k-1} / I_{k-1}\right) \otimes_{A} E_{2} \rightarrow\left(J_{k-1} / I_{k-1}\right) \otimes_{A} O
$$

be the obvious homomorphism. By definition, $\beta\left(f_{k}\right)$ and $\beta\left(g_{k}\right)$ are the obstruction classes to extending $\Phi_{k-1}=\Psi_{k-1}: \operatorname{Spec}_{A} M / J_{k-1} \rightarrow Z$ to $\operatorname{Spec}_{A} M / I_{k-1}$. It follows that $\beta\left(f_{k}\right)=\beta\left(g_{k}\right)$, and hence

$$
f_{k}-g_{k} \in\left(J_{k-1} / I_{k-1}\right) \otimes_{A} \operatorname{Im}\left\{E_{1} \rightarrow E_{2}\right\} .
$$

Let $t \in M \otimes_{A} E_{2}$ be a lift of $f_{k}-g_{k}$. Then $F_{k}-G_{k}-t \in I_{k-1} \otimes_{A} E_{2}$. On the other hand, since $M \otimes_{A} \operatorname{Im}\left\{E_{1} \rightarrow E_{2}\right\} \subset J_{1} \otimes_{A} E_{2} \subset M_{1} \otimes_{A} E_{2}$,

$$
t \in\left(J_{1} \cdot J_{k-1}\right) \otimes_{A} E_{2} \subset I_{k-1} \otimes_{A} E_{2} .
$$

Therefore $f_{k}-g_{k} \in I_{k-1} \otimes_{A} E_{2}$. This implies that for some $\eta \in \operatorname{Hom}_{A}\left(E_{2}, M \otimes_{A} E_{2}\right)$ satisfying property $(2.1)$, we have $G-\eta(F) \equiv 0 \bmod M_{k+1}$. Hence $J_{k}=(F)+$ $M_{k+1}=(G)+M_{k+1}$.

Next we analyze $\Phi_{k}$ and $\Psi_{k}$. Let $\varphi_{k}, \psi_{k}: \Gamma\left(\mathcal{O}_{Z}\right) \longrightarrow M / J_{k}$ be the homomorphisms of rings induced by $\Phi_{k}$ and $\Psi_{k}$ respectively. Then since $\varphi_{k} \equiv \psi_{k}$ $\bmod M_{k}$, there is a $D \in \operatorname{Der}_{A}\left(\Gamma\left(\mathcal{O}_{Z}\right), J_{k-1} / J_{k}\right)$ such that $\psi_{k}=\varphi_{k}+D$ (see [Ma, p. 191]). Since $\left(J_{k-1} / J_{k}\right) \cdot M_{1}=0$ and since $\Phi_{k}$ induces an isomorphism between $\Omega_{Z / S} \otimes_{\mathcal{O}_{Z}} \mathcal{O}_{S}$ and $\Omega_{Y / S} \otimes_{\mathcal{O}_{Y}} \mathcal{O}_{S}$, where $Y=\operatorname{Spec}_{A} M / J_{k}$, there is a $D_{0} \in \operatorname{Der}_{A}\left(M / J_{k}, J_{k-1} / J_{k}\right)$ so that $\psi_{k}=\left(\mathrm{id}+D_{0}\right) \circ \phi_{k}$. Since id $+D_{0}$ is an isomorphism of $M / J_{k}$ that is the identity modulo $M_{k}$, there is an isomorphism $\xi: M \rightarrow M$ so that $\xi \equiv 1_{M} \bmod M_{k}, \xi\left(J_{k}\right)=J_{k}$ and the induced homomorphism $M / J_{k} \rightarrow M / J_{k}$ is exactly id $+D_{0}$.

The transformation $(\xi, \eta)$ is not quite what we want, since it satisfies the relations

$$
G \equiv \eta(F) \quad \bmod M_{k+1} \quad \text { and } \quad \Psi \equiv \Phi \circ \bar{\xi} \bmod M_{k+1} .
$$

To obtain $\eta$ so that $G=\left(\left(1_{M} \otimes \eta\right) \circ\left(\xi \otimes 1_{E_{2}}\right)\right)(F)$, we instead look at the relative Kuranishi family $\left(F^{\prime}, \Phi^{\prime}\right)=\left(\xi, 1_{E_{2}}\right)(F, \Phi)$. Then since

$$
\left(F^{\prime}, \Phi^{\prime}\right) \equiv(F, \Phi) \equiv(G, \Psi) \bmod M_{k},
$$

by the previous argument we can find an $\eta \in \operatorname{Hom}_{A}\left(E_{2}, M \otimes_{A} E_{2}\right)$ satisfying (2.1) so that $G \equiv \eta\left(F^{\prime}\right) \bmod M_{k+1}$.

Now we apply induction on $k$. The previous argument shows that there is a sequence of transformations $T_{k} \in \mathcal{K}$ so that if we let $S_{k}=T_{k} \circ \cdots \circ T_{2}$, then $S_{k}(F, \Phi) \equiv(G, \Psi) \bmod M_{k+1}$. Let $T_{k}$ be $\left(\xi_{k}, \eta_{k}\right)$. Since $\xi_{k} \equiv 1_{M} \bmod M_{k}$, $\xi_{k} \circ \cdots \circ \xi_{2}$ converges to an automorphism $\xi_{\infty}: M \rightarrow M$. $\xi_{\infty}$ satisfies the property (2.1). Now let $\left(F^{\prime}, \Phi^{\prime}\right)=\left(\xi_{\infty}, 1_{E_{2}}\right)(F, \Phi)$. For the same reason, there is a sequence $\eta_{k} \in \operatorname{Hom}_{A}\left(E_{2}, M_{1} \otimes_{A} E_{2}\right)$ so that if we let $H_{1}=F^{\prime}$ and $H_{k+1}=\left(1_{E_{2}}+\eta_{k}\right)\left(H_{k}\right)$, then $G \equiv H_{k+1} \bmod M_{k+1}$. Applying the Artin-Rees lemma to the ideal $L=$ $\left(F^{\prime}\right) \subset M$, we can find an integer $c$ so that

$$
L M_{1} \cap M_{1}^{n}=M_{1}^{n-c}\left(L M_{1} \cap M_{1}^{c}\right)
$$


for any $n>c$. Let $L_{k}=\left(H_{k}\right)$. Since $L$ is isomorphic to $L_{k}$ under an isomorphism of $M$, the same identity holds with $L$ replaced by $L_{k}$. Then since

$$
\eta_{k}\left(H_{k}\right)=H_{k+1}-H_{k} \in \operatorname{Hom}_{A}\left(E_{2}, E_{2}\right) \otimes_{A}\left(L_{k} M_{1} \cap M_{1}^{k}\right),
$$

for $k \geq c$, we can assume that the $\eta_{k}$ have already been chosen so that

$$
\eta_{k} \in \operatorname{Hom}_{A}\left(E_{2}, E_{2}\right) \otimes_{A} M_{1}^{k-c} .
$$

With this choice of $\eta_{k}$, the composite $\left(1_{E_{2}}+\eta_{k}\right) \cdots\left(1_{E_{2}}+\eta_{1}\right)$ will converge to an $\eta_{\infty} \in \operatorname{Hom}_{A}\left(E_{2}, M \otimes_{A} E_{2}\right)$ such that $\eta_{\infty} \equiv 1_{E_{2}} \bmod M_{1}$ and $G=\eta_{\infty}\left(F^{\prime}\right)$. Therefore, $\left(\xi_{\infty}, \eta_{\infty}\right)(F, \Phi)=(G, \Psi)$. This proves Lemma 2.6 and Proposition 2.4.

Corollary 2.7. Let $Z / S$ and $\mathcal{T}_{Z / S}=\mathfrak{h} \bullet\left(\mathcal{E}^{\bullet}\right)$ be as in the situation of Proposition 2.4. Let $T \subset S$ be a closed subscheme. Let $W=Z \times{ }_{S} T$ and $B=\Gamma\left(\mathcal{O}_{T}\right)$. Then $\mathcal{T}_{Z / S}$ canonically induces a relative perfect tangent-obstruction complex $\mathcal{T}_{W / T}^{\bullet}=$ $\mathfrak{h}^{\bullet}\left(\mathcal{E} \bullet \otimes_{\mathcal{O}_{S}} \mathcal{O}_{T}\right)$. Further, if $(F, \Phi)$ is a relative Kuranishi family of $\mathcal{T}_{Z / S}$, then $F^{\prime}=F \otimes_{A} B$ and the restriction of $\Phi$ to Spec $N /\left(F^{\prime}\right)$, where $N=M \otimes_{A} B$, is a relative Kuranishi family of $\mathcal{T}_{W / S}^{\bullet}=\mathfrak{h}^{\bullet}\left(\mathcal{E}^{\bullet} \otimes_{\mathcal{O}_{S}} \mathcal{O}_{T}\right)$.

Proof. This is obvious from the proof of Lemma 2.5.

Before we close this section, we will point out the relation between the relative Kuranishi families and the Kuranishi families in the usual sense. Let $S$ and $\mathcal{T}_{S}^{\bullet}=$ $\mathfrak{h}^{\bullet}\left(\mathcal{E}^{\bullet}\right)$ be an affine scheme and a perfect tangent-obstruction complex of $S$. Let $Z$ be the formal completion of $S \times S$ along its diagonal and let $\mathcal{T}_{Z / S}=\mathfrak{h}^{\bullet}\left(\mathcal{E}^{\bullet}\right)$ be its induced perfect relative tangent-obstruction complex. Let $(F, \Phi)$ be a relative Kuranishi family of this complex. In the following, we will localize $(F, \Phi)$ and compare it with the usual Kuranishi maps.

Let $q \in S$ be any closed point and let $\mathfrak{m} \subset A$ be the maximal ideal of $q \in S$. Let $\hat{A}=\lim A / \mathfrak{m}^{n}$, let $\hat{E}_{i}=E_{i} \otimes_{A} \hat{A}$ and let $\hat{M}=M \otimes_{A} \hat{A}$ be their respective formal completions. We denote by $\hat{Z}$ the formal completion of $Z$ along $Z \times{ }_{S}\{q\}$ and by $\hat{S}$ the formal completion of $S$ along $q$. Then $\mathcal{T}_{Z / S}^{\bullet}=\mathfrak{h}^{\bullet}\left(\mathcal{E}^{\bullet}\right)$ canonically induces a perfect relative tangent-obstruction complex $\mathcal{T}_{\dot{Z} / \hat{S}}^{\bullet}=\mathfrak{h}^{\bullet}\left(\hat{E}^{\bullet}\right)$. Obviously, $(F, \Phi)$ induces a Kuranishi family

$$
\hat{F} \in \hat{M} \otimes_{\hat{A}} \hat{E}_{2} \quad \text { and } \quad \hat{\Phi}: \operatorname{Spec} \hat{M} /(\hat{F}) \stackrel{\cong}{\longrightarrow} \hat{Z}
$$

of $\mathcal{T}_{\dot{Z} / \hat{S}}=\mathfrak{h}^{\bullet}\left(\hat{E}^{\bullet}\right)$.

Now we turn to the usual Kuranishi families. For simplicity, we assume $\mathcal{E}_{2} \otimes_{\mathcal{O}_{S}}$ $k(q)$ is isomorphic to $\mathfrak{h}^{2}\left(\mathcal{E}^{\bullet}\right) \otimes_{\mathcal{O}_{S}} k(q)$. Let $T_{i}=\mathcal{E}_{i} \otimes_{\mathcal{O}_{S}} k(q)$. Then $T_{1}$ is the tangent space $T_{q} S$. The complex $\mathcal{T}_{S}^{\bullet}$ induces an obstruction theory to deformations of $q$ in $S$ taking values in $T_{2}$. Now let $B=\hat{\operatorname{Sym}} \bullet\left(T_{1}^{\vee}\right)$ and let

$$
f \in B \otimes_{k} T_{2}^{\vee} \quad \text { and } \quad \varphi: \operatorname{Spec} B /(f) \stackrel{\cong}{\longrightarrow} \hat{S}
$$

be a Kuranishi family (cf. [La]). In the following, we will construct a pair $(\hat{f}, \hat{\varphi})$ from $(f, \varphi)$ analogous to $(\hat{F}, \hat{\Phi})$. Let $I \subset B \otimes_{k} B$ be the ideal generated by $a \otimes 1-1 \otimes a$ and let $\hat{B}=\lim B \otimes_{k} B / I^{n}$. Let $p_{1}, p_{2}: B \rightarrow \hat{B}$ be the homomorphisms defined by $p_{1}(a)=a \otimes 1$ and $p_{2}(a)=1 \otimes a$. For $f \in B \otimes_{k} T_{2}$ given before, we denote by $p_{1}(f)$ the image of $f$ under $p_{1} \otimes 1_{T_{2}}: B \otimes T_{2} \rightarrow \hat{B} \otimes_{k} T_{2}$. As before, we denote by $\left(p_{1}(f)\right) \subset \hat{B}$ the ideal generated by the components of $p_{1}(f)$. We let $C=\hat{B} /\left(p_{1}(f)\right)$. $C$ is an 
$\hat{A}$-algebra via $\hat{A}=B /(f) \rightarrow C$ induced by $p_{1}$. Let $\hat{f}=p_{2}(f)$. It follows that Spec $C /(\hat{f})$ is the formal completion of $\hat{S} \times \hat{S}$ along its diagonal. Because $\hat{Z}$ is the formal completion of $\hat{S} \times \hat{S}$ along its diagonal, we obtain a canonical $\hat{S}$-isomorphism

$$
\hat{\varphi}: \operatorname{Spec} C /(\hat{f}) \stackrel{\cong}{\longrightarrow} \text {. }
$$

Now let $I_{C} \subset C$ be the ideal generated by the images of $I \subset B \otimes_{k} B$. Note that $C / I_{C} \cong \hat{A}$. We consider the complex of $\hat{A}$-modules

$$
\hat{F}^{\bullet}=\left[\left(I_{C} / I_{C}^{2}\right)^{\vee} \longrightarrow T_{2} \otimes_{k} \hat{A}\right]
$$

indexed at 1 and 2, where the arrow is the dual of $d \hat{f}: T_{2}^{\vee} \otimes \hat{A} \rightarrow I_{C} / I_{C}^{2}$. Since $H^{-1}\left(\operatorname{Hom}_{\hat{A}}\left(\hat{F}^{\bullet}, \hat{A}\right)\right)=\Omega_{B /(f)}$, there is an isomorphism of complexes

$$
\operatorname{Hom}_{\hat{A}}\left(\hat{E}^{\bullet}, \hat{A}\right) \stackrel{\cong}{\longrightarrow} \operatorname{Hom}_{\hat{A}}\left(\hat{F}^{\bullet}, \hat{A}\right)
$$

so that their induced isomorphism on $H^{-1}$ is the canonical isomorphism between $\Omega_{\hat{S}}$ and $\Omega_{B /(f)}$. Namely, we have the commutative diagram

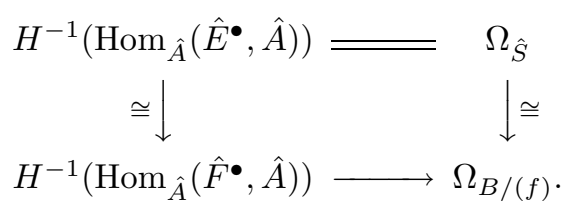

Let $\left(r_{1}, r_{2}\right): \hat{E}^{\bullet} \rightarrow \hat{F}^{\bullet}$, where $r_{i}: \hat{E}_{i} \rightarrow \hat{F}_{i}$ are the corresponding isomorphisms. Since $\hat{F}^{\bullet} \otimes_{\hat{A}} k$ and $\hat{E}^{\bullet} \otimes_{\hat{A}} k$ are $T_{1} \stackrel{\times 0}{\rightarrow} T_{2}$, we can choose $r_{2}$ so that its tensoring with $k$ is the identity of $T_{2}$. We now compare the pairs $(\hat{f}, \hat{\varphi})$ and $(\hat{F}, \hat{\Phi})$. Let $\xi_{1}: C \rightarrow \hat{M}$ be the $\hat{A}$-isomorphism induced by the dual of $r_{1}: \hat{E}_{1} \rightarrow\left(I_{C} / I_{C}^{2}\right)^{\vee}$ and let $\eta_{1}: T_{2} \otimes_{k} \hat{A} \rightarrow \hat{E}_{2}$ be $r_{2}^{-1}$. Then our choice of $r_{1}$ and $r_{2}$ guarantees that

$$
\left(\left(1_{\hat{M}} \otimes \eta_{1}\right) \circ\left(\xi_{1} \otimes 1_{T_{2}}\right)\right)(\hat{f}) \equiv \hat{F} \quad \bmod \hat{M}_{2} \quad \text { and } \quad \hat{\varphi} \circ \bar{\xi}_{1} \equiv \hat{\Phi} \quad \bmod \hat{M}_{2},
$$

where $\bar{\xi}_{1}: \operatorname{Spec} \hat{M} /(\hat{F}) \rightarrow \operatorname{Spec} C /(\hat{f})$ is the isomorphism induced by $\xi_{1}$.

Lemma 2.8. There is an $\hat{A}$-isomorphism $\xi: C \rightarrow \hat{M}$ and

$$
\eta \in \operatorname{Hom}_{\hat{A}}\left(T_{2} \otimes_{k} \hat{A}, \hat{M} \otimes_{\hat{A}} \hat{E}_{2}\right)
$$

satisfying

$$
\xi \equiv \xi_{1} \quad \bmod \hat{M}_{2} \quad \text { and } \quad \eta \equiv \eta_{1} \quad \bmod \hat{M}_{1}
$$

such that

$$
\left(\left(1_{\hat{M}} \otimes \eta\right) \circ\left(\xi \otimes 1_{T_{2}}\right)\right)(\hat{f})=\hat{F} \quad \text { and } \quad \hat{\varphi} \circ \bar{\xi}=\hat{\Phi},
$$

where, as usual, $\bar{\xi}$ is the isomorphism induced by $\xi$.

Proof. The proof is parallel to that of Lemma 2.6. The difference is that in this case we can only compare the obstruction classes when they lie in $\mathfrak{h}^{2}\left(\hat{E}^{\bullet} \otimes_{\hat{A}} k\right)$ or in $\mathfrak{h}^{2}\left(\hat{F}^{\bullet} \otimes_{\hat{A}} k\right)$, because the identification (2.4) is canonical only after tensoring $k$. We proceed as follows. Let $\mathfrak{m}_{0} \subset C$ and $\mathfrak{m}_{0}^{\prime} \subset \hat{M}$ be their maximal ideals, and let $J_{k}=I_{C}^{2} \cdot \mathfrak{m}_{0}^{k-1} \subset C$ and $J_{k}^{\prime}=\hat{M}_{2} \cdot \mathfrak{m}_{0}^{\prime k-1} \subset \hat{M}$. Assume that there are $\xi_{k-1}: C \rightarrow \hat{M}$ and $\eta_{k-1} \in \operatorname{Hom}_{\hat{A}}\left(T_{2} \otimes_{k} \hat{A}, \hat{M} \otimes_{\hat{A}} \hat{E}_{2}\right)$ satisfying (2.5) such that $\left(\left(1_{\hat{M}} \otimes \eta_{k-1}\right) \circ\left(\xi_{k-1} \otimes 1_{T_{2}}\right)\right)(\hat{f}) \equiv \hat{F} \quad \bmod J_{k}^{\prime} \quad$ and $\quad \hat{\varphi} \circ \bar{\xi}_{k-1} \equiv \hat{\Phi} \quad \bmod J_{k}^{\prime}$. 
We consider the residue classes

$$
\begin{aligned}
& o_{k}=\text { residue of } \hat{f} \text { in }\left(\left((\hat{f})+J_{k}\right) /\left((\hat{f}) \cdot \mathfrak{m}_{0}+J_{k+1}\right)\right) \otimes_{k} T_{2} ; \\
& o_{k}^{\prime}=\text { residue of } \hat{F} \text { in }\left(\left((\hat{F})+J_{k}^{\prime}\right) /\left((\hat{F}) \cdot \mathfrak{m}_{0}^{\prime}+J_{k+1}^{\prime}\right)\right) \otimes_{k} T_{2} .
\end{aligned}
$$

Because $f$ and $F$ are the (relative) Kuranishi maps, they are the obstruction class to lifting

$$
\hat{A} \stackrel{p_{2}}{\longrightarrow} \mathcal{O}_{\hat{Z}} \longrightarrow C /\left((\hat{f})+J_{k}\right) \quad \text { to } \quad \hat{A} \rightarrow C /\left((\hat{f}) \cdot \mathfrak{m}_{0}+J_{k+1}\right)
$$

and the obstruction class to lifting

$$
\hat{A} \stackrel{p_{2}}{\longrightarrow} \mathcal{O}_{\hat{Z}} \longrightarrow \hat{M} /\left((\hat{F})+J_{k}^{\prime}\right) \quad \text { to } \quad \hat{A} \rightarrow \hat{M} /\left((\hat{F}) \cdot \mathfrak{m}_{0}^{\prime}+J_{k+1}^{\prime}\right),
$$

where $p_{2}$ is induced by $Z \rightarrow S \times S \stackrel{\mathrm{pr}_{2}}{\longrightarrow} S$. It follows that they must coincide under the isomorphism

$$
\left(\left((\hat{f})+J_{k}\right) /\left((\hat{f}) \cdot \mathfrak{m}_{0}+J_{k+1}\right)\right) \otimes_{k} T_{2} \cong\left(\left((\hat{F})+J_{k}^{\prime}\right) /\left((\hat{F}) \cdot \mathfrak{m}_{0}^{\prime}+J_{k+1}^{\prime}\right)\right) \otimes_{k} T_{2}
$$

induced by $\xi_{k-1}$. The remainder argument is a repetition of the proof of Lemma 2.6 , and will be omitted. This proves the lemma.

\section{ViRTuAl NORMAL CONES}

In the first part of this section, for any quasi-projective scheme $W$ and a perfect tangent-obstruction complex $\mathcal{T}_{W}^{\bullet}=\mathfrak{h}^{\bullet}\left(\mathcal{E}^{\bullet}\right)$, we will construct a virtual normal cone $C^{\mathcal{E}^{\bullet}} \subset \operatorname{Vect}\left(\mathcal{E}_{2}\right)$. Here $\operatorname{Vect}\left(\mathcal{E}_{2}\right)$ is the vector bundle on $W$ so that its sheaf of sections is $\mathcal{E}_{2}$. By abuse of notation, we will not distinguish a vector bundle with the scheme of its total space. The cone $C^{\mathcal{E}^{\bullet}}$ will be the restriction to $W$ of the normal cone to the zero locus of a relative Kuranishi map in its graph. Based on the property of the relative Kuranishi families, we will show that $C^{\mathcal{E}^{\bullet}}$ is unique (as a scheme). Because of this, this construction can be applied to the moduli functors represented by Deligne-Mumford moduli stacks.

We begin with an affine scheme $S$ and a perfect tangent-obstruction complex $\mathcal{T}_{S}^{\bullet}=\mathfrak{h}^{\bullet}\left(\mathcal{E}^{\bullet}\right)$. Let $Z$ be the formal completion of $S \times S$ along its diagonal. We continue to use the convention adopted in the previous section. Namely, $A=\Gamma\left(\mathcal{O}_{S}\right)$, $E_{i}=\Gamma\left(\mathcal{E}_{i}\right)$, which are assumed to be free $A$-modules, and $M=\hat{\operatorname{Sym}} \bullet\left(E_{1}^{\vee}\right)$. We let $N=\operatorname{Sym} \bullet\left(E_{2}^{\vee}\right)$. Let $(F, \Phi)$, where $F \in M \otimes_{A} E_{2}$, be a relative Kuranishi family of $\mathcal{T}_{Z / S}^{\bullet}=\mathfrak{h}^{\bullet}\left(\mathcal{E}^{\bullet}\right)$. It is clear that $F$ extends to an $A$-homomorphism $N \rightarrow M$ of $A$-algebras. We let $\Gamma_{F} \subset \operatorname{Spec} N \otimes_{A} M$ be its graph. We let $j: S \rightarrow \operatorname{Spec} N$ be the obvious section and let

$$
\iota=j \times_{S} 1: \operatorname{Spec} M=S \times_{S} \operatorname{Spec} M \rightarrow \operatorname{Spec} N \otimes_{A} M .
$$

We view $\iota$ as the 0 -section of $\operatorname{Spec} N \otimes_{A} M \rightarrow \operatorname{Spec} M$. In the following, we will view $Z$ as a subscheme of $\operatorname{Spec} M$ via the isomorphism $\operatorname{Spec} M /(F) \cong Z$. It follows that

$$
\iota(Z)=\Gamma_{F} \times_{\operatorname{Spec}} N \otimes_{A} M \iota(\operatorname{Spec} M) .
$$

We let $\mathcal{N}^{F}$ be the normal cone to $\iota(Z)$ in $\Gamma_{F} \cdot \mathcal{N}^{F}$ is canonically embedded as a closed subcone in $\operatorname{Vect}\left(\mathcal{E}_{2}\right) \times_{S} Z$, which is the normal bundle to $\iota(\operatorname{Spec} M)$ in Spec $N \otimes_{A} M$. Finally, we let $C^{\mathcal{E}^{\bullet}}$ be the restriction of $\mathcal{N}^{F}$ to $S$ :

$$
C^{\mathcal{E}^{\bullet}}=\mathcal{N}^{F} \times{ }_{Z} S .
$$


Remark. The cone $\mathcal{N}^{F}$ is the normal cone to $Z$ in Spec $M$, denoted $C_{Z / \operatorname{Spec} M}$, and $C^{\mathcal{E}^{\bullet}}=C_{Z / \operatorname{Spec} M} \times{ }_{Z} S$. However, using the graph description it is clear how these cones are canonically embedded in the vector bundles $\operatorname{Vect}\left(\mathcal{E}_{2}\right) \times_{S} Z$ and $\operatorname{Vect}\left(\mathcal{E}_{2}\right)$, respectively.

Lemma 3.1. Let $(F, \Phi)$ and $(G, \Psi)$ be two relative Kuranishi families of $\mathcal{T}_{Z / S}=$ $\mathfrak{h}^{\bullet}\left(\mathcal{E}^{\bullet}\right)$. Then as subschemes of $\operatorname{Vect}\left(\mathcal{E}_{2}\right) \times{ }_{S} Z$,

$$
\mathcal{N}^{F} \times{ }_{Z} S=\mathcal{N}^{G} \times{ }_{Z} S .
$$

In particular, the cone $C^{\mathcal{E}^{\bullet}} \subset \operatorname{Vect}\left(\mathcal{E}_{2}\right)$ does not depend on the choice of the relative Kuranishi families.

Proof. By Proposition 2.4, there is a transformation $(\xi, \eta) \in \mathcal{H}$ so that $(\xi, \eta)(F, \Phi)=$ $(G, \Psi)$. Let $\theta$ be the automorphism of $N \otimes_{A} M$ defined by

$$
a \otimes 1 \mapsto \eta^{i n v}(a) \quad \text { and } \quad 1 \otimes b \mapsto 1 \otimes \xi(b) .
$$

Here, $\eta^{i n v}: N \rightarrow N \otimes_{A} M$ is the homomorphism induced by

$$
\eta^{-1} \in \operatorname{Hom}_{A}\left(E_{2}, M \otimes_{A} E_{2}\right)
$$

such that $\eta^{-1} \circ \eta=1_{E_{2}}$. Let $\bar{\theta}: \operatorname{Spec} N \otimes_{A} M \rightarrow \operatorname{Spec} N \otimes_{A} M$ be the induced isomorphism. Clearly, $\bar{\theta}$ preserves $\iota(\operatorname{Spec} M)$ and induces an isomorphism between $\Gamma_{F}$ and $\Gamma_{G}$. Hence $\bar{\theta}$ induces an isomorphism, denoted $\bar{\theta}_{*}$, of the normal bundle to $\iota(\operatorname{Spec} M)$ in Spec $N \otimes_{A} M$ with itself. It follows that $\bar{\theta}_{*}$ induces an isomorphism between $\mathcal{N}^{F}$ and $\mathcal{N}^{G}$. Finally, because $\eta \equiv 1_{E_{2}} \bmod M_{1}$ and $\xi \equiv 1_{M} \bmod M_{2}$, the restriction of $\bar{\theta}_{*}$ to $\operatorname{Vect}\left(\mathcal{E}_{2}\right) \subset \operatorname{Vect}\left(\mathcal{E}_{2}\right) \times_{S} Z$ is the identity homomorphism. Therefore, $\mathcal{N}^{F} \times{ }_{Z} S=\mathcal{N}^{G} \times{ }_{Z} S$. This proves the lemma.

We will call $C^{\mathcal{E}^{\bullet}} \subset \operatorname{Vect}\left(\mathcal{E}_{2}\right)$ the virtual normal cone of the tangent-obstruction complex $\mathcal{T}_{\dot{S}}^{\bullet}=\mathfrak{h} \bullet(\mathcal{E} \bullet$.

Lemma 3.2. Let the notation be as before. Assume $\mathcal{F}^{\bullet}=\left[\mathcal{F}_{1} \rightarrow \mathcal{F}_{2}\right]$ is another complex of locally free sheaves so that $\mathcal{T}_{S}^{\bullet}=\mathfrak{h}^{\bullet}\left(\mathcal{F}^{\bullet}\right)$. Assume further that there is a surjective homomorphism of complexes $\mathcal{F}^{\bullet} \rightarrow \mathcal{E}^{\bullet}$ such that the induced isomorphism of their sheaf cohomologies $\mathfrak{h}^{\bullet}\left(\mathcal{F}^{\bullet}\right) \cong \mathfrak{h}^{\bullet}\left(\mathcal{E}^{\bullet}\right)$ is the identity, using the isomorphisms $\mathfrak{h}^{\bullet}\left(\mathcal{F}^{\bullet}\right)=\mathcal{T}_{S}^{\bullet}=\mathfrak{h}^{\bullet}\left(\mathcal{E}^{\bullet}\right)$. Let $\varphi_{2}: \mathcal{F}_{2} \rightarrow \mathcal{E}_{2}$ be one of the homomorphisms and let $C\left(\varphi_{2}\right): \operatorname{Vect}\left(\mathcal{F}_{2}\right) \rightarrow \operatorname{Vect}\left(\mathcal{E}_{2}\right)$ be the induced submersive morphism. Then

$$
C\left(\varphi_{2}\right)^{-1}\left(C^{\mathcal{E}^{\bullet}}\right)=C^{\mathcal{F}^{\bullet}} .
$$

Here by $C\left(\varphi_{2}\right)^{-1}\left(C^{\mathcal{E}^{\bullet}}\right)$ we mean $C^{\mathcal{E}^{\bullet}} \times \operatorname{Vect}\left(\mathcal{E}_{2}\right) \operatorname{Vect}\left(\mathcal{F}_{2}\right)$.

Proof. This is a local problem. By shrinking $S$ if necessary, we can assume that there is an isomorphism $\mathcal{F}^{\bullet} \cong \mathcal{E}^{\bullet} \oplus\left[\mathcal{O}_{S}^{\oplus a} \stackrel{\text { Id }}{\rightarrow} \mathcal{O}_{S}^{\oplus a}\right]$ so that the given $\mathcal{F}^{\bullet} \rightarrow \mathcal{E}^{\bullet}$ is the obvious projection. Let $F_{1}: E_{2}^{\vee} \rightarrow M$ be a relative Kuranishi family of $\mathcal{T}_{Z / S}=\mathfrak{h}^{\bullet}\left(\mathcal{E}^{\bullet}\right)$. Let $M^{\prime}=\hat{\operatorname{Sym}} \bullet\left(A^{\oplus a}\right)$ and let $F_{2}: A^{\oplus a} \rightarrow M^{\prime}$ be induced by id: $A^{\oplus a} \rightarrow A^{\oplus a}$. Then

$$
F_{1} \otimes 1+1 \otimes F_{2}: E_{2}^{\vee} \oplus A^{\oplus a} \longrightarrow M \otimes M^{\prime}
$$

is a relative Kuranishi family of $\mathcal{T}_{Z / S}^{\bullet}=\mathfrak{h}^{\bullet}\left(\mathcal{F}^{\bullet}\right)$. A direct computation on normal cones shows that $C^{\mathcal{F}^{\bullet}}$ is the pull back of $C^{\mathcal{E}^{\bullet}}$ under the obvious projection $\operatorname{Vect}\left(\mathcal{F}_{2}\right) \rightarrow \operatorname{Vect}\left(\mathcal{E}_{2}\right)$. This proves that $C\left(\varphi_{2}\right)^{-1}\left(C^{\mathcal{E}^{\bullet}}\right)=C^{\mathcal{F}^{\bullet}}$. 
Let $q \in S$ be any closed point, let $T_{1}=T_{q} S$ and $T_{2}=\mathfrak{h}^{2}\left(\mathcal{E}^{\bullet}\right) \otimes_{\mathcal{O}_{S}} k(q)$. Then $\mathcal{T}_{S}$ provides an obstruction theory to deformations of $q$ in $S$. As in section 2, we let $\hat{S}$ be the formal completion of $S$ at $q$, let $f: T_{2}^{\vee} \rightarrow B$, where $B=\hat{\operatorname{Sym}} \bullet\left(T_{1}^{\vee}\right)$, be a Kuranishi map. Let $C^{f}$ be the normal cone to $\operatorname{Spec} B /(f)$ in Spec $B$. It follows from the above Remark that $C^{f}$ is canonically embedded in $\operatorname{Vect}\left(T_{2}\right) \times_{k} \hat{S}$.

Lemma 3.3. Let the notation be as above. Then there is a quotient vector bundle map $j: \operatorname{Vect}\left(\mathcal{E}_{2}\right) \times_{S} \hat{S} \rightarrow \operatorname{Vect}\left(T_{2}\right) \times \hat{S}$ extending the given quotient map

$$
\operatorname{Vect}\left(\mathcal{E}_{2}\right) \times_{S}\{q\}=\operatorname{Vect}\left(\mathcal{E}_{2} \otimes_{\mathcal{O}_{S}} k(q)\right) \rightarrow \operatorname{Vect}\left(T_{2}\right)
$$

so that as subschemes in $\operatorname{Vect}\left(\mathcal{E}_{2}\right) \times_{S} \hat{S}$,

$$
\left(\operatorname{Vect}\left(\mathcal{E}_{2}\right) \times{ }_{S} \hat{S}\right) \times_{\operatorname{Vect}\left(T_{2}\right) \times \hat{S}} C^{f}=\hat{S} \times{ }_{S} C^{\mathcal{E}} .
$$

Proof. We first consider the case where $\operatorname{dim} T_{2}=\operatorname{rank} \mathcal{E}_{2}$. Then we are in the situation of Lemma 2.8 and its proof. We continue to use the notations introduced there. Let $R_{1}=\operatorname{Spec} \hat{M}$, let $i_{1}: \hat{S} \rightarrow R_{1}$ be the obvious section induced by $S \rightarrow$ Spec $M$ and let $\hat{F} \in \hat{M} \otimes_{\hat{A}} \hat{E}_{2}$ be the image of $F$ under $M \otimes_{A} E_{2} \rightarrow \hat{M} \otimes_{\hat{A}} \hat{E}_{2}$. We let $R_{2}=\operatorname{Spec} C \subset \operatorname{Spec} \hat{B}, i_{2}: \hat{S} \rightarrow R_{2}$ be the section induced by $a \otimes b \mapsto a b$ and let $\hat{f} \in C \otimes_{k} T_{2}$ be the image of the Kuranishi map $f: T_{2}^{\vee} \rightarrow B$ under

$$
B \otimes_{k} T_{2} \stackrel{p_{2} \times 1_{T_{2}}}{\longrightarrow} B \otimes_{k} B \otimes_{k} T_{2} \longrightarrow C \otimes_{k} T_{2},
$$

where $p_{2}(a)=1 \otimes a$. Let $V_{1}=\operatorname{Spec}\left(\hat{\operatorname{Sym}} \bullet\left(\hat{E}_{2}^{\vee}\right)\right)$ and $V_{2}=\operatorname{Spec}\left(\hat{\operatorname{Sym}} \bullet\left(T_{2}^{\vee} \otimes \hat{A}\right)\right)$. Let $0_{V_{i}}$ be the 0 -section of $V_{i} \rightarrow \hat{S}$. In the proof of Lemma 2.8, we have shown that there is an isomorphism

$$
K: V_{1} \times_{\hat{S}} R_{1} \longrightarrow V_{2} \times_{\hat{S}} R_{2}
$$

for which the following holds. First, it induces an isomorphism between $0_{V_{1}} \times_{\hat{S}} R_{1}$ and $0_{V_{2}} \times{ }_{\hat{S}} R_{2}$, and induces an isomorphism between the graphs $\Gamma_{\hat{F}}$ and $\Gamma_{\hat{f}}$. Second, let

$$
\varphi:\left(\operatorname{Vect}\left(\mathcal{E}_{2}\right) \times{ }_{S} \hat{S}\right) \times{ }_{\hat{S}} R_{1} \longrightarrow \operatorname{Vect}\left(T_{2}\right) \times R_{2}
$$

be the induced isomorphism between the normal bundle to $0_{V_{1} \times{ }_{\hat{S}}} R_{1}$ in $V_{1} \times{ }_{\hat{S}} R_{1}$ and the normal bundle to $0_{V_{2}} \times{ }_{\hat{S}} R_{2}$ in $V_{2} \times{ }_{\hat{S}} R_{2}$. Then the restriction of $\varphi$ to the fiber over the closed point of $R_{1}$ is the identity homomorphism between $\operatorname{Vect}\left(\mathcal{E}_{2}\right) \times_{S}\{q\}=$ $\operatorname{Vect}\left(\mathcal{E}_{2} \otimes_{\mathcal{O}_{S}} k(q)\right)$ and $\operatorname{Vect}\left(T_{2}\right)$.

Now let $\mathcal{N}_{1}$ be the normal cone to Spec $\hat{M} /(\hat{F})$ in $R_{1}$ and let $\mathcal{N}_{2}$ be the normal cone to Spec $C /(\hat{f})$ in $R_{2}$. Note that $\mathcal{N}_{1}$ and $\mathcal{N}_{2}$ are canonically embedded in $V_{1} \times{ }_{\hat{S}}$ Spec $\hat{M} /(\hat{F})$ and in $V_{2} \times{ }_{\hat{S}} \operatorname{Spec} C /(\hat{f})$, respectively. Let $\bar{\varphi}$ be the restriction of $\varphi$ to $\operatorname{Vect}\left(\mathcal{E}_{2}\right) \times{ }_{S} \operatorname{Spec} \hat{M} /(\hat{F}) . \bar{\varphi}$ is an isomorphism between $\operatorname{Vect}\left(\mathcal{E}_{2}\right) \times_{{ }_{S}} \operatorname{Spec} \hat{M} /(\hat{F})$ and $\operatorname{Vect}\left(T_{2}\right) \times \operatorname{Spec} C /(\hat{f})$. Since $K$ preserves the 0 -sections and the graphs, $\bar{\varphi}\left(\mathcal{N}_{1}\right)=$ $\mathcal{N}_{2}$ and hence

$$
\bar{\varphi}\left(\mathcal{N}_{1} \times_{\operatorname{Spec} \hat{M}} \hat{S}\right)=\mathcal{N}_{2} \times_{\operatorname{Spec} C} \hat{S} .
$$

Since the term inside the parentheses on the left hand side is $C^{\mathcal{E}^{\bullet}} \times{ }_{S} \hat{S}$, to prove the lemma, it suffices to show that the right hand side is $C^{f}$. Let $\pi$ : Spec $C \rightarrow \operatorname{Spec} B$ be the morphism induced by $a \mapsto 1 \otimes a$. Clearly, $\pi$ is flat and

$$
\operatorname{Spec} C \times_{\operatorname{Spec} B} \operatorname{Spec} B /(f)=\operatorname{Spec} C /(\hat{f}) \text {. }
$$


It follows from [Vi, p. 639] that $C^{f} \times_{\operatorname{Spec} B} \operatorname{Spec} C=\mathcal{N}_{2}$. Therefore

$$
\mathcal{N}_{2} \times_{\operatorname{Spec} C} \hat{S}=C^{f} \times_{\operatorname{Spec} B} \operatorname{Spec} C \times \times_{\operatorname{Spec} C} \hat{S}=C^{f} .
$$

This proves the lemma in case $\operatorname{rank} \mathcal{E}_{2}=\operatorname{dim} T_{2}$. In general, by shrinking $S$ if necessary we can find a complex $\mathcal{F}^{\bullet}$ and an isomorphism of complexes $\mathcal{E}^{\bullet} \cong \mathcal{F}^{\bullet} \oplus$ $\left[\hat{A}^{\oplus a} \stackrel{\text { id }}{\rightarrow} \hat{A}^{\oplus a}\right]$ such that $a=\operatorname{rank} \mathcal{E}_{2}-\operatorname{dim} T_{2}$. Then the general case follows from Lemma 3.2 and the situation just proved. This proves the lemma.

Since $\operatorname{dim} \Gamma_{f}=\operatorname{rank} \mathcal{E}_{1}$, we have $\operatorname{dim} C^{f}=\operatorname{rank} \mathcal{E}_{1}$. This proves

Corollary 3.4. The cone $C^{\mathcal{E}^{\bullet}}$ is equidimensional and has dimension rank $\mathcal{E}_{1}$.

Corollary 3.5. Assume that we have two complexes $\mathcal{E}^{\bullet}$ and $\mathcal{F}^{\bullet}$ so that $\mathfrak{h}^{\bullet}\left(\mathcal{E}^{\bullet}\right)=$ $\mathcal{T}_{\dot{S}}^{\bullet}$ and $\mathfrak{h}^{\bullet}\left(\mathcal{F}^{\bullet}\right)=\mathcal{T}_{S}^{\bullet}$. Assume further that there is a surjective homomorphism $\varphi: \mathcal{F}_{2} \rightarrow \mathcal{E}_{2}$ so that the following diagram is commutative:

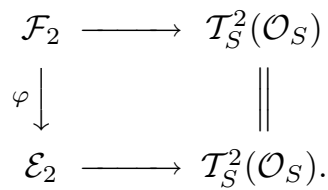

Then as cycles, we have

$$
C(\varphi)^{*}\left(\left[C^{\mathcal{E}^{\bullet}}\right]\right)=\left[{C^{\mathcal{F}}}^{\bullet}\right] \in Z_{*}\left(\operatorname{Vect}\left(\mathcal{F}_{2}\right)\right) .
$$

Here $C(\varphi): \operatorname{Vect}\left(\mathcal{F}_{2}\right) \rightarrow \operatorname{Vect}\left(\mathcal{E}_{2}\right)$ is the induced morphism on vector bundles.

Proof. Let $q \in S$ be any closed point. Lemma 3.3 says that there are quotient vector bundle homomorphisms

$j_{1}: \operatorname{Vect}\left(\mathcal{E}_{2}\right) \times_{S} \hat{S} \longrightarrow \operatorname{Vect}\left(T_{2}\right) \times \hat{S} \quad$ and $\quad j_{2}: \operatorname{Vect}\left(\mathcal{F}_{2}\right) \times_{S} \hat{S} \longrightarrow \operatorname{Vect}\left(T_{2}\right) \times \hat{S}$, extending the given $\operatorname{Vect}\left(\mathcal{E}_{2}\right) \times{ }_{S}\{q\} \rightarrow \operatorname{Vect}\left(T_{2}\right)$ and $\operatorname{Vect}\left(\mathcal{F}_{2}\right) \times_{S}\{q\} \rightarrow \operatorname{Vect}\left(T_{2}\right)$ respectively, such that $j_{1}^{-1}\left(C^{f}\right)=C^{\mathcal{E}^{\bullet}} \times_{S} \hat{S}$ and $j_{2}^{-1}\left(C^{f}\right)=C^{\mathcal{F}^{\bullet}} \times{ }_{S} \hat{S}$. It follows that there is a vector bundle quotient homomorphism $j: \operatorname{Vect}\left(\mathcal{F}_{2}\right) \times_{S} \hat{S} \rightarrow \operatorname{Vect}\left(\mathcal{E}_{2}\right) \times_{S} \hat{S}$ extending $\operatorname{Vect}\left(\mathcal{F}_{2}\right) \times_{S}\{q\} \rightarrow \operatorname{Vect}\left(\mathcal{E}_{2}\right) \times_{S}\{q\}$ such that $j^{-1}\left(C^{\mathcal{E}^{\bullet}} \times{ }_{S} \hat{S}\right)=C^{\mathcal{F}^{\bullet}} \times{ }_{S} \hat{S}$. This implies that cycles $\left[C^{\mathcal{F}^{\bullet}}\right]$ and $C(\varphi)^{*}\left(\left[C^{\mathcal{E}^{\bullet}}\right]\right)$ have the same support along the fiber over $q$ and that the multiplicities of their respective components near the fiber over $q$ coincide. Since $q$ is arbitrary, we must have $C(\varphi)^{*}\left(\left[C^{\mathcal{E}^{\bullet}}\right]\right)=\left[C^{\mathcal{F}^{\bullet}}\right]$. This proves the corollary.

Remark. The proof shows that the cycle $\left[C^{\mathcal{E}^{\bullet}}\right]$ can be characterized as follows. At each $q \in S$, there is a quotient vector bundle homomorphism

$$
j: \operatorname{Vect}\left(\mathcal{E}_{2}\right) \times_{S} \hat{S} \rightarrow \operatorname{Vect}\left(T_{2}\right) \times \hat{S},
$$

extending $\operatorname{Vect}\left(\mathcal{E}_{2}\right) \times_{S}\{q\} \rightarrow \operatorname{Vect}\left(T_{2}\right)$, such that $j^{*}\left(\left[C^{f}\right]\right)=r^{*}\left[C^{\mathcal{E}^{\bullet}}\right]$, where $r$ : $\operatorname{Vect}\left(\mathcal{E}_{2}\right) \times{ }_{S} \hat{S} \rightarrow \operatorname{Vect}\left(\mathcal{E}_{2}\right)$ is the induced morphism and is flat. Clearly, this criterion determines $\left[C^{\mathcal{E}^{\bullet}}\right]$ completely, if it exists. The reason we need to use the relative Kuranishi families is to ensure that $\left[C^{\mathcal{E}^{\bullet}}\right]$ does exist as a cycle.

Let $S_{0} \subset S$ be a closed subscheme. Then $\mathcal{T}_{Z / S}=\mathfrak{h}^{\bullet}\left(\mathcal{E}^{\bullet}\right)$ induces canonically a relative tangent-obstruction complex $\mathcal{T}_{Z \times_{S} S_{0} / S_{0}}=\mathfrak{h}^{\bullet}\left(\mathcal{F}^{\bullet}\right)$, where $\mathcal{F}^{\bullet}=\mathcal{E}^{\bullet} \otimes_{\mathcal{O}_{S}} \mathcal{O}_{S_{0}}$. Let $(F, \Phi)$ be a Kuranishi family of $\mathcal{T}_{Z / S}^{\bullet}=\mathfrak{h} \bullet\left(\mathcal{E}^{\bullet}\right)$. Let $A_{0}=\Gamma\left(\mathcal{O}_{S_{0}}\right), M_{0}=$ $M \otimes_{A} A_{0}$ and $F_{i}=E_{i} \otimes_{A} A_{0}$. The pair $F_{0} \in M_{0} \otimes_{A_{0}} F_{2}$ and $\Phi_{0}: \operatorname{Spec} M_{0} /\left(F_{0}\right) \rightarrow$ $Z \times_{S} S_{0}$ defined by $F_{0}=F \otimes_{A} A_{0}$ and $\Phi_{0}=\left.\Phi\right|_{\operatorname{Spec} M_{0} /\left(F_{0}\right)}$ is a relative Kuranishi 
family of $\mathcal{T}_{Z \times{ }_{S} S_{0} / S_{0}}=\mathfrak{h}^{\bullet}\left(\mathcal{F}^{\bullet}\right)$. We let $\mathcal{C}=C_{\left(\operatorname{Spec} M_{0} /\left(F_{0}\right)\right) / \operatorname{Spec} M_{0}}$ and set $C^{\mathcal{F}^{\bullet}}=$ $\mathcal{C} \times$ Spec $M_{0} S_{0}$, which is canonically embedded in $\operatorname{Vect}\left(\mathcal{F}_{2}\right)$. Note that $\operatorname{Vect}\left(\mathcal{F}_{2}\right)=$ $\operatorname{Vect}\left(\mathcal{E}_{2}\right) \times{ }_{S} S_{0}$.

Corollary 3.6. $C^{\mathcal{F}^{\bullet}}=C^{\mathcal{E}^{\bullet}} \times{ }_{S} S_{0}$ as subschemes of $\operatorname{Vect}\left(\mathcal{F}_{2}\right)$.

Proof. The proof is similar to the proof of Corollary 3.5. We continue to use the notations introduced there. Let $q \in S_{0}$ be any closed point and let $\hat{S}_{0}$ be the formal completion of $S_{0}$ along $q$. It follows that $\hat{S}_{0}=\hat{S} \times{ }_{S} S_{0}$. Let $j_{1}$ be the map constructed in the proof of Corollary 3.5 and let $j_{2}$ be the restriction of $j_{1}$ to $\operatorname{Vect}\left(\mathcal{E}_{2}\right) \times_{S} \hat{S}_{0}$. Hence, $j_{2}: \operatorname{Vect}\left(\mathcal{F}_{2}\right) \times_{S_{0}} \hat{S}_{0} \rightarrow \operatorname{Vect}\left(T_{2}\right) \times \hat{S}_{0}$. Then Lemma 3.3 shows that $j_{1}^{-1}\left(C^{f}\right)=C^{\mathcal{E}^{\bullet}} \times{ }_{S} \hat{S}$. We claim that $j_{2}^{-1}\left(C^{f}\right)=C^{\mathcal{F}^{\bullet}} \times{ }_{S_{0}} \hat{S}_{0}$. Indeed, by the proof of Lemma 3.3, it suffices to check that if we let $C_{0}$ be the formal completion of $M_{0}$ along the maximal ideal $\mathfrak{m}_{0}$ of $q \in S_{0}$, then $C_{0}$ is flat over $B$ via the homomorphism $B \rightarrow C_{0}$ induced by $p_{2}$. But this is obvious. This proves the claim. Therefore,

$$
C^{\mathcal{E}^{\bullet}} \times{ }_{S} \hat{S}_{0}=j_{1}^{-1}\left(C^{f}\right) \times{ }_{\hat{S}} \hat{S}_{0}=j_{2}^{-1}\left(C^{f}\right)=C^{\mathcal{F}^{\bullet}} \times{ }_{S_{0}} \hat{S}_{0} .
$$

This proves that $C^{\mathcal{F}^{\bullet}}=C^{\mathcal{E}^{\bullet}} \times{ }_{S} S_{0}$.

In the remainder of this section, we will construct the virtual normal cone and the virtual cycle of a perfect tangent-obstruction complex. We will show in the end that this construction commutes with the refined Gysin maps.

Let $Z$ be a quasi-projective scheme and let $\mathcal{T}_{Z}^{\bullet}$ be a tangent-obstruction complex of $Z$. We assume that $\mathcal{E}^{\bullet}=\left[\mathcal{E}_{1} \rightarrow \mathcal{E}_{2}\right]$ is a complex of locally free sheaves of $\mathcal{O}_{Z^{-}}$ modules so that $\mathcal{T}_{Z}=\mathfrak{h}^{\bullet}\left(\mathcal{E}^{\bullet}\right)$. We cover $Z$ by affine open $S_{\alpha}$ such that $\Gamma_{S_{\alpha}}\left(\mathcal{E}_{i}\right)$ are free $\Gamma\left(\mathcal{O}_{S_{\alpha}}\right)$-modules for $i=1,2$. It follows from Lemma 3.2 that we have canonical cones $C_{\alpha}^{\mathcal{E}^{\alpha}} \subset \operatorname{Vect}\left(\mathcal{E}_{2}\right) \times_{Z} S_{\alpha}$ of the tangent-obstruction complex

$$
\mathcal{T}_{S_{\alpha}}^{\bullet}=\mathfrak{h}^{\bullet}\left(\mathcal{E}^{\bullet} \otimes_{\mathcal{O}_{X}} \mathcal{O}_{S_{\alpha}}\right) .
$$

By Lemma 3.3, $S_{\alpha} \times{ }_{Z} C_{\beta}^{\mathcal{E}^{\bullet}}=S_{\beta} \times{ }_{Z} C_{\alpha}^{\mathcal{E}^{\bullet}}$ as subcones in $\operatorname{Vect}\left(\mathcal{E}_{2}\right) \times{ }_{Z}\left(S_{\alpha} \cap S_{\beta}\right)$. Therefore $C_{\alpha}^{\mathcal{E}^{\bullet}}$ patch together to form a global cone scheme $C^{\mathcal{E}^{\bullet}} \subset \operatorname{Vect}\left(\mathcal{E}_{2}\right)$.

We remark that a global resolution $\mathcal{T}_{Z}^{\bullet}=\mathfrak{h}^{\bullet}\left(\mathcal{E}^{\bullet}\right)$ allows us to construct a global cone as a subscheme in $\operatorname{Vect}\left(\mathcal{E}_{2}\right)$. However, if we only have a locally free sheaf $\mathcal{V}$ making $\mathcal{T}_{Z}^{2}\left(\mathcal{O}_{Z}\right)$ its quotient sheaf, then we can canonically construct a cone cycle as follows. Since $\mathcal{T}_{Z}^{\bullet}$ is perfect, we can find an open covering $S_{\alpha}$ of $Z$ and complexes $\mathcal{E}_{\alpha}^{\bullet}$ of sheaves of $\mathcal{O}_{S_{\alpha}}$-modules such that $\mathfrak{h}^{\bullet}\left(\mathcal{E}_{\alpha}^{\bullet}\right)=\mathcal{T}_{\dot{S}_{\alpha}}$ and that there are quotient homomorphisms $\varphi_{\alpha}: \mathcal{V} \otimes_{\mathcal{O}_{Z}} \mathcal{O}_{S_{\alpha}} \rightarrow \mathcal{E}_{2, \alpha}$ such that

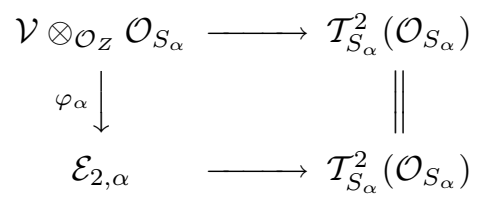

is commutative. Because of Corollary 3.5, the flat pull backs $C\left(\varphi_{\alpha}\right)^{*}\left(\left[C^{\mathcal{E}_{\alpha}^{\bullet}}\right]\right)$ and $C\left(\varphi_{\beta}\right)^{*}\left(\left[C^{\mathcal{E}_{\beta}^{\bullet}}\right]\right)$ coincide over $S_{\alpha} \cap S_{\beta}$. Therefore they patch together to form a cycle $\left[C^{\mathcal{V}}\right] \in Z_{*} \operatorname{Vect}(\mathcal{V})$. Because of Corollary 3.5 again, $\left[C^{\mathcal{V}}\right]$ is unique.

Now we construct the virtual cycle of a perfect tangent-obstruction complex $\mathcal{T}_{Z}$. We first present $\mathcal{T}_{Z}^{2}\left(\mathcal{O}_{Z}\right)$ as a quotient sheaf of a locally free sheaf $\mathcal{V}$, which is possible since $Z$ is quasi-projective. Let $i_{V}: Z \rightarrow \operatorname{Vect}(\mathcal{V})$ be the zero section and let $i_{V}^{*}: A_{*}(\operatorname{Vect}(\mathcal{V})) \rightarrow A_{*} Z$ be the Gysin homomorphism. 
Definition 3.7. Let the notation be as before. Then we define the virtual cycle $[Z]^{\text {vir }}$ of $\mathcal{T}_{Z}^{\bullet}$ to be

$$
[Z]^{\mathrm{vir}}=i_{V}^{*}\left[C^{\mathcal{V}}\right] \in A_{*} Z .
$$

In order to show that $[Z]^{\text {vir }}$ is well-defined, we need to check that it is independent of the choice of quotient homomorphism $\mathcal{V} \rightarrow \mathcal{T}_{Z}^{2}\left(\mathcal{O}_{Z}\right)$. Assume that $\mathcal{W}$ is another locally free sheaf of $\mathcal{O}_{Z}$-modules and $\mathcal{W} \rightarrow \mathcal{T}_{Z}^{2}\left(\mathcal{O}_{Z}\right)$ is a quotient homomorphism. We let $\mathcal{M}^{\prime}$ be the pull back defined by the square

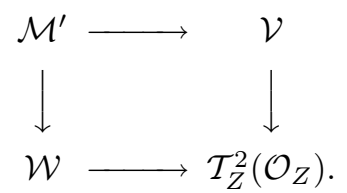

Then by making $\mathcal{M}^{\prime}$ a quotient sheaf of a locally free sheaf, say $\mathcal{M}$, we obtain $\phi_{1}: \mathcal{M} \rightarrow \mathcal{V}$ and $\phi_{2}: \mathcal{M} \rightarrow \mathcal{W}$. It follows from Corollary 3.5 that

$$
C\left(\phi_{1}\right)^{*}\left(\left[C^{\mathcal{V}}\right]\right)=\left[C^{\mathcal{M}}\right]=C\left(\phi_{2}\right)^{*}\left(\left[C^{\mathcal{W}}\right]\right) .
$$

This implies that $i_{V}^{*}\left[C^{\mathcal{V}}\right]=i_{M}^{*}\left[C^{\mathcal{M}}\right]=i_{W}^{*}\left[C^{\mathcal{W}}\right] \in A_{*} Z$ as required. So $[Z]^{\text {vir }}$ is well-defined.

Refined Gysin maps play an important role in intersection theory. Given a fiber product square of schemes

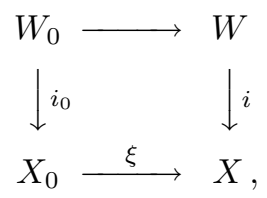

where $\xi$ is a regular embedding of codimension $d$, then the refined Gysin map

$$
\xi^{!}: A_{*} W \longrightarrow A_{*-d} W_{0}
$$

sends $D \in A_{*} W$ to the intersection of $\left[C_{D \times X_{X} X_{0} / D}\right]$ with the zero section of $i_{0}^{*} N_{X_{0} / X}$. In this section, we will show that the refined Gysin map is compatible to our virtual cycle construction.

Let $W$ be a quasi-projective scheme over $X$ and let $X_{0} \subset X$ be a regular embedding. We define $W_{0}$ by the Cartesian square (3.1). We assume that $W$ (resp. $W_{0}$ ) admits a perfect tangent-obstruction complex $\mathcal{T}_{W}^{\bullet}\left(\right.$ resp. $\mathcal{T}_{W_{0}}^{\bullet}$ ). Let $\mathcal{L}$ be the sheaf of normal bundle to $X_{0}$ in $X$. For any affine $S, \eta: S \rightarrow W_{0} \subset W$ and $\mathcal{F} \in \mathfrak{M o d}_{S}$, there is a canonical sheaf homomorphism

$$
\mathcal{T}_{W}^{1}(\eta)(\mathcal{F})=\mathcal{H o m}_{\mathcal{O}_{S}}\left(\eta^{*} \Omega_{W}, \mathcal{F}\right) \longrightarrow\left(i_{0} \circ \eta\right)^{*} \mathcal{L} \otimes_{\mathcal{O}_{S}} \mathcal{F},
$$

induced by $i^{*} \mathcal{I}_{X_{0} \subset X} \rightarrow \mathcal{I}_{W_{0} \subset W}$, that fits into the exact sequence

$$
0 \longrightarrow \mathcal{T}_{W_{0}}^{1}(\eta)(\mathcal{F}) \longrightarrow \mathcal{T}_{W}^{1}(\eta)(\mathcal{F}) \longrightarrow\left(i_{0} \circ \eta\right)^{*} \mathcal{L} \otimes_{\mathcal{O}_{S}} \mathcal{F}
$$

Definition 3.8. We say that $\mathcal{T}_{W}^{\bullet}$ and $\mathcal{T}_{W_{0}}$ are compatible with the Cartesian square (3.1) if (3.2) extends to a long exact sequence

$$
\mathcal{T}_{W}^{1}(\eta)(\mathcal{F}) \longrightarrow\left(i_{0} \circ \eta\right)^{*} \mathcal{L} \otimes_{\mathcal{O}_{S}} \mathcal{F} \stackrel{\delta}{\longrightarrow} \mathcal{T}_{W_{0}}^{2}(\eta)(\mathcal{F}) \stackrel{r}{\longrightarrow} \mathcal{T}_{W}^{2}(\eta)(\mathcal{F}) \longrightarrow 0
$$

for which the following holds. Let $S \rightarrow Y_{0} \rightarrow Y$ be a tuple of $S$-schemes described in Definition 1.2 and let $\mathcal{I}=\mathcal{I}_{Y_{0} \subset Y}$. Let $\eta_{0}: Y_{0} \rightarrow W_{0}$ be any morphism and let $o \in \mathcal{T}_{W_{0}}^{2}\left(\eta_{0}\right)(\mathcal{I})$ be the obstruction class to extending $\eta_{0}$ to $Y \rightarrow W_{0}$. Then $r(o) \in$ $\mathcal{T}_{W}^{2}\left(\eta_{0}\right)(\mathcal{I})$ is the obstruction class to extending $\eta_{0}$ to $Y \rightarrow W$. Secondly, assume 
that $r(o)=0$. Then we have an extension, say $\eta: Y \rightarrow W$. Let $\beta \in\left(i_{0} \circ \eta_{0}\right)^{*} \mathcal{L} \otimes_{\mathcal{O}_{S}} \mathcal{I}$ be the canonical homomorphism $\left(i_{0} \circ \eta_{0}\right)^{*}\left(\mathcal{I}_{X_{0} \subset X} / \mathcal{I}_{X_{0} \subset X}^{2}\right) \rightarrow \mathcal{I}$ induced by $\eta$. Then $o=\delta(\beta)$.

We make one technical assumption, which usually can be checked explicitly in applications. Let $S_{\alpha}$ be an open covering of $W_{0}$. We assume that there are complexes of locally free sheaves $\mathcal{E}_{\alpha}^{\bullet}$ and $\mathcal{F}_{\alpha}^{\bullet}$ of $\mathcal{O}_{S_{\alpha}}$-modules fitting into the exact sequence

$$
0 \longrightarrow\left[0 \rightarrow i_{0}^{*} \mathcal{L} \otimes_{W_{0}} \mathcal{O}_{S_{\alpha}}\right] \longrightarrow \mathcal{E}_{\alpha}^{\bullet} \longrightarrow \mathcal{F}_{\alpha}^{\bullet} \longrightarrow 0
$$

such that $\mathcal{T}_{S_{\alpha}}^{\bullet}=\mathfrak{h}^{\bullet}\left(\mathcal{E}_{\alpha}^{\bullet}\right), \mathcal{T}_{S_{\alpha}}^{\bullet}=\mathfrak{h}^{\bullet}\left(\mathcal{F}_{\alpha}^{\bullet}\right)$ and the long exact sequence of sheaf cohomologies

$$
\mathfrak{h}^{j}\left(\left[0 \rightarrow i_{0}^{*} \mathcal{L} \otimes_{W_{0}} \mathcal{O}_{S_{\alpha}}\right]\right) \longrightarrow \mathfrak{h}^{j}\left(\mathcal{E}_{\alpha}^{\bullet}\right) \longrightarrow \mathfrak{h}^{j}\left(\mathcal{F}_{\alpha}^{\bullet}\right) \longrightarrow \mathfrak{h}^{j+1}\left(\left[0 \rightarrow i_{0}^{*} \mathcal{L} \otimes_{W_{0}} \mathcal{O}_{S_{\alpha}}\right]\right)
$$

is the exact sequence given in the above definition. We assume that there are sheaves $\mathcal{E}_{2}$ and $\mathcal{F}_{2}$ of $\mathcal{O}_{W_{0}}$-modules so that $\mathcal{E}_{\alpha, 2}$ and $\mathcal{F}_{\alpha, 2}$ are restrictions of $\mathcal{E}_{2}$ and $\mathcal{F}_{2}$ to $S_{\alpha}$ respectively. Also, over $S_{\alpha} \cap S_{\beta}$, there are isomorphisms of $\mathcal{E}_{\alpha}^{\bullet}$ and $\mathcal{E}_{\beta}^{\bullet}$ and isomorphisms of $\mathcal{F}_{\alpha}^{\bullet}$ and $\mathcal{F}_{\beta}^{\bullet}$ so that the induced isomorphisms on their sheaf cohomologies are the identity maps. Finally, we assume that $\mathcal{F}_{2}$ can be extended to a sheaf of $\mathcal{O}_{W}$-modules, say $\tilde{\mathcal{F}}_{2}$, so that $\mathcal{F}_{2} \rightarrow \mathcal{T}_{W_{0}}^{2}\left(\mathcal{O}_{W_{0}}\right)$ extends to a quotient homomorphism $\tilde{\mathcal{F}}_{2} \rightarrow \mathcal{T}_{W}^{2}\left(\mathcal{O}_{W}\right)$.

Proposition 3.9. Let $W_{0} \subset W$ be defined by the square (3.1) such that their tangent-obstruction complexes $\mathcal{T}_{W_{0}}^{\bullet}$ and $\mathcal{T}_{W}^{\bullet}$ are compatible. Assume further that the technical conditions stated above are satisfied. Let $\left[W_{0}\right]^{\text {vir }}$ and $[W]^{\text {vir }}$ be the virtual cycles of $\mathcal{T}_{W_{0}}^{\bullet}$ and $\mathcal{T}_{W}^{\bullet}$ respectively. Then

$$
\xi^{!}[W]^{\mathrm{vir}}=\left[W_{0}\right]^{\mathrm{vir}}
$$

This identity is essentially a statement about associativity of refined Gysin maps. As usual, we will transform this problem to a problem about the commutativity of Gysin maps and then apply the basic Lemma in [Vi] to conclude the proof of the proposition. We now provide the details of the proof, which will occupy the rest of this section.

We first introduce some notations. Let $\hat{W}_{0}$ (resp. $\hat{W}$ ) be the formal completion of $W_{0} \times W_{0}$ (resp. $W \times W$ ) along its diagonal, considered as a scheme over $W_{0}$ (resp. $W$ ) via the first projection of the product. We denote by $p_{W_{0}}: \hat{W}_{0} \rightarrow W_{0}$ (resp. $p_{W}: \hat{W} \rightarrow W$ ) the morphism induced by the second projection. We begin with a locally closed affine subscheme $S \subset S_{\alpha}$. We fix the complexes $\mathcal{E}_{\alpha}^{\bullet}$ and $\mathcal{F}_{\alpha}^{\bullet}$ given before. We let $A=\Gamma\left(\mathcal{O}_{S}\right), F_{i}=\Gamma\left(\mathcal{F}_{\alpha, i} \otimes_{\mathcal{O}_{W_{0}}} \mathcal{O}_{S}\right), E_{i}=\Gamma\left(\mathcal{E}_{\alpha, i} \otimes_{\mathcal{O}_{W_{0}}} \mathcal{O}_{S}\right)$ and $L=\Gamma\left(i_{0}^{*} \mathcal{L} \otimes \mathcal{O}_{W_{0}} \mathcal{O}_{S}\right)$. By shrinking $S_{\alpha}$ if necessary, we can assume that all modules $F_{i}, E_{i}$ and $L$ are free $A$-modules. As before, we let $M=\hat{\operatorname{Sym}} \bullet\left(E_{1}^{\vee}\right)$, which is canonically isomorphic to $\hat{\operatorname{Sym}} \bullet\left(F_{i}^{\vee}\right)$ using $\mathcal{E}_{\alpha, 1} \cong \mathcal{F}_{\alpha, 1}$. We pick a relative Kuranishi family $(f, \varphi)$ of $\mathcal{T}_{\dot{W} \times_{W} S / S}$, where $f \in M \otimes_{A} F_{2}$ and $\varphi$ is an $S$-isomorphism Spec $M /(f) \rightarrow \hat{W} \times_{W} S$. We now pick a relative Kuranishi family of $\mathcal{T}_{\hat{W}_{0} \times W_{0} S / S}$.

We first pick a splitting $\sigma: F_{2} \rightarrow E_{2}$ of the exact sequence $L \rightarrow E_{2} \rightarrow F_{2}$. Let $g_{1}=\left(1_{M} \otimes \sigma\right)(f)$. Note that $\left(g_{1}\right)=(f)$, hence Spec $M /\left(g_{1}\right)$ is isomorphic to $\hat{W} \times{ }_{W} S$. We denote this isomorphism by $\varphi$. Let $l=\operatorname{codim}\left(X_{0}, X\right)$. Without loss of generality, we can assume that near $i(S) \subset X$ the sheaf $\mathcal{I}_{X_{0} \subset X}$ is generated by $l$ sections, say $s_{1}, \cdots, s_{l}$. We let $\bar{s}_{1}, \ldots, \bar{s}_{l} \in M /\left(g_{1}\right)$ be the pull backs of $s_{1}, \cdots, s_{l}$ 
via

$$
\text { Spec } M /\left(g_{1}\right) \longrightarrow \hat{W} \times_{W} S \stackrel{\mathrm{pr}_{1}}{\longrightarrow} \hat{W} \stackrel{p_{W}}{\longrightarrow} W \stackrel{i}{\longrightarrow} X .
$$

Note that $\left(s_{1}, \cdots, s_{l}\right)$ form a basis of $\mathcal{I}_{X_{0} \subset X} / \mathcal{I}_{X_{0} \subset X}^{2}$ near $i(S)$. Then $\left(\bar{s}_{1}, \cdots, \bar{s}_{l}\right)$ defines a homomorphism $\bar{g}_{2}: L^{\vee} \rightarrow M /\left(g_{1}\right)$. Let $\phi: \operatorname{Spec} M /\left(g_{1}, \bar{g}_{2}\right) \rightarrow \hat{W}_{0} \times_{W_{0}} S$ be the morphism induced by $\varphi: \operatorname{Spec} M /\left(g_{1}\right) \rightarrow \hat{W} \times{ }_{W} S$.

Lemma 3.10. Let $\tau: M \otimes_{A} L \rightarrow M \otimes_{A} E_{2}$ be the homomorphism induced by $L \rightarrow E_{2}$. Then there is a lift $g_{2} \in M \otimes_{A} L$ of $\bar{g}_{2} \in\left(M /\left(g_{1}\right)\right) \otimes_{A} L$ such that

$$
g:=g_{1}+\tau\left(g_{2}\right) \in M \otimes_{A} E_{2} \quad \text { and } \quad \phi: \operatorname{Spec} M /(g) \longrightarrow \hat{W}_{0} \times_{W_{0}} S
$$

form a relative Kuranishi family of $\mathcal{T}_{\dot{W}_{0} \times W_{0} S / S}=\mathfrak{h}^{\bullet}\left(\mathcal{E}_{\alpha}^{\bullet} \otimes_{\mathcal{O}_{W_{0}}} \mathcal{O}_{S}\right)$.

Proof. Assume that we have found a lift $h \in M \otimes_{A} L$ of $\bar{g}_{2}$ such that $g_{1}+\tau(h)$ $\bmod M_{k}$ and the above $\phi$ satisfy the property in Lemma 2.5. Let $J=\left(g_{1}+\tau(h)\right) \subset$ $M, J_{k-1}=J+M_{k}$ and $I_{k-1}=J \cdot M_{1}+M_{k+1}$. We consider the epimorphism $M / J_{k-1} \rightarrow M / I_{k-1}$ and its kernel $J_{k-1} / I_{k-1}$. Now let $O=\mathcal{T}_{W}^{2}\left(\mathcal{O}_{S}\right)$, let $O_{0}=$ $\mathcal{T}_{W_{0}}^{2}\left(\mathcal{O}_{S}\right)$ and let $r: O_{0} \rightarrow O$ be the homomorphism given in Definition 3.8. Let $o$ (resp. $o_{0}$ ) be the obstruction class to extending

$$
\varphi_{k}=\left.\varphi\right|_{\text {Spec } M / J_{k-1}}: \operatorname{Spec} M / J_{k-1} \longrightarrow \hat{W} \times_{W} S
$$

to $\operatorname{Spec} M / I_{k-1} \rightarrow \hat{W} \times_{W} S$ (resp. to Spec $M / I_{k-1} \rightarrow \hat{W}_{0} \times_{W_{0}} S$ ). Since the obstructions are compatible, we have that $r\left(o_{0}\right)=o$. Now let $\bar{g}_{1}$ and $\bar{\tau}(h)$ be the residue classes of $g_{1}$ and $\tau(h)$ in $\left(J_{k-1} / I_{k-1}\right) \otimes_{A} O_{0}$, respectively. Since $f$ is a relative Kuranishi map, we have that $r\left(\bar{g}_{1}\right)=o$. On the other hand, we know that $\varphi_{k}$ extends to Spec $M /\left(I_{k-1}+(f)\right) \rightarrow \hat{W} \times_{W} S$. It follows that the residue class of $\tau(h)$ in $\left(J_{k-1} /\left(I_{k-1}+(f)\right)\right) \otimes_{A} O_{0}$, which also is the residue class of $\bar{g}_{2}$, is the obstruction class to extending $\varphi_{k}$ to

$$
\text { Spec } M /\left(I_{k-1}+(f)\right) \longrightarrow \hat{W}_{0} \times_{W_{0}} S \text {. }
$$

Therefore $\left(\bar{g}_{1}+\bar{\sigma}(h)\right)-o_{0}$ belongs to

$$
\operatorname{Ker}\left\{\left(J_{k-1} / I_{k-1}\right) \otimes_{A} O_{0} \longrightarrow\left(J_{k-1} / I_{k-1}\right) \otimes_{A} O \oplus\left(J_{k-1} /\left(I_{k-1}+(f)\right)\right) \otimes_{A} O_{0}\right\} .
$$

This proves that there is an $\epsilon_{k} \in(f) \otimes_{A} L$ so that $g_{1}+\tau\left(h+\epsilon_{k}\right) \bmod M_{k+1}$ satisfies the property in Lemma 2.5. It follows from the proof of Lemma 2.5 that we can choose $\epsilon_{k}$ to be in $\left((f) \cap M_{k}\right) \otimes_{A} L$. Hence an induction on $k$ shows that there is a lift $g_{2} \in M \otimes_{A} L$ such that $g:=g_{1}+\tau\left(g_{2}\right) \in M \otimes_{A} E_{2}$ and $\phi=\left.\varphi\right|_{\operatorname{Spec} M /(g)}$ is a relative Kuranishi family of $\mathcal{T}_{\dot{W}_{0} \times_{W_{0}} S / S}=\mathfrak{h}^{\bullet}\left(\mathcal{E}^{\bullet} \otimes_{\mathcal{O}_{W_{0}}} \mathcal{O}_{S}\right)$.

Now let $Z=\operatorname{Spec} M$ and let $Z(g)=\operatorname{Spec} M /(g) \subset Z$. Then $Z$ is a scheme over $S$, thus a scheme over $W_{0}$. Let $V_{1}=\operatorname{Vect}(\mathcal{L}) \times_{W_{0}} Z, V=\operatorname{Vect}\left(\mathcal{E}_{2}\right) \times_{W_{0}} Z$ and $V_{2}=\operatorname{Vect}\left(\mathcal{F}_{2}\right) \times_{W_{0}} Z$. Then $V_{1}$ is a subbundle of $V$ and $V_{2}$ is the quotient vector bundle $V / V_{1}$. Let $C_{Z(g) / Z}$ be the normal cone to $Z(g)$ in $Z$. The cone $C_{Z(g) / Z}$ is canonically embedded in $V \times{ }_{Z} Z(g)$. We let

$$
D_{1}(S)=C_{Z /(g) / Z} \times{ }_{Z} S \subset V \times{ }_{Z} S .
$$

It follows from Lemma 3.1 that for the affine covering $S_{\alpha}$ of $W_{0}$, the collection $\left\{D_{1}\left(S_{\alpha}\right)\right\}$ patches together to form a cone $\mathbf{D}_{1}$ in $V$. By Definition 3.7, if we let $\eta_{1}: W_{0} \rightarrow V$ be the zero section,

$$
\left[W_{0}\right]^{\text {vir }}=\eta_{1}^{*}\left[\mathbf{D}_{1}\right] .
$$


Next, we consider the subscheme $Z(f)=$ Spec $M /(f) \subset Z$ and the normal cone $C_{2}=C_{Z(f) / Z}$, which is naturally a subcone of $V_{2} \times_{Z} Z(f)$. We let $C_{2} \rightarrow X$ be the morphism induced by

$$
V_{2} \times_{Z} Z(f) \stackrel{\mathrm{pr}_{2}}{\longrightarrow} Z(f)=\hat{W} \times_{W} S \stackrel{\mathrm{pr}_{1}}{\longrightarrow} \hat{W} \stackrel{p_{W}}{\longrightarrow} W \stackrel{i}{\longrightarrow} X .
$$

The normal cone $C_{C_{2} \times X_{X} X_{0} / C_{2}}$ is canonically a subcone in

$$
\left(V_{2} \times_{Z}\left(\hat{W} \times_{W} S\right)\right) \times_{Z}\left(V_{1} \times_{Z}\left(\hat{W}_{0} \times_{W_{0}} S\right)\right)=\left(V_{1} \times_{Z} V_{2}\right) \times_{Z}\left(\hat{W}_{0} \times_{W_{0}} S\right) .
$$

We set

$$
D_{2}(S)=C_{C_{2} \times X_{X} X_{0} / C_{2}} \times_{\hat{W}_{0} \times W_{0} S} S .
$$

For the same reason, the collection $\left\{D_{2}\left(S_{\alpha}\right)\right\}$ patch together to form a cone $\mathbf{D}_{2} \subset$ $\operatorname{Vect}(\mathcal{L}) \times_{W_{0}} \operatorname{Vect}\left(\mathcal{F}_{2}\right)$. We claim that

$$
\xi^{!}[W]^{\mathrm{vir}}=\eta_{2}^{*}\left[\mathbf{D}_{2}\right],
$$

where $\eta_{2}: W_{0} \rightarrow V_{1} \times_{W_{0}} V_{2}$ is the zero section. By our technical assumption, $\mathcal{F}_{2}$ extends to $\tilde{\mathcal{F}}_{2}$ so that $\mathcal{F}_{2} \rightarrow \mathcal{T}^{2} \mathcal{F}_{W_{0}}\left(\mathcal{O}_{W_{0}}\right)$ extends to $\tilde{\mathcal{F}}_{2} \rightarrow \mathcal{T}_{W}^{2}\left(\mathcal{O}_{W}\right)$. Let $\mathcal{N} \in Z_{*} \operatorname{Vect}\left(\tilde{\mathcal{F}}_{2}\right)$ be the virtual cone cycle of $\mathcal{T}_{W}$ provided by Lemma 3.1 and Corollary 3.5. Then the normal cone cycle $\left[C_{\mathcal{N} \times{ }_{X} X_{0} / \mathcal{N}}\right]$ is canonically a cone cycle in $\operatorname{Vect}(\mathcal{L}) \times_{W_{0}} \operatorname{Vect}\left(\mathcal{F}_{2}\right)$. It follows from $\left[\mathrm{Vi}\right.$, p. 643] that $\xi^{*}[W]^{\mathrm{vir}}=\eta_{2}^{*}\left[C_{\mathcal{N} \times_{X} X_{0} / \mathcal{N}}\right]$. However, by using Lemma 3.1 and Corollary 3.6, we have that $\left[C_{\mathcal{N} \times{ }_{X} X_{0} / \mathcal{N}}\right]=\left[\mathbf{D}_{2}\right]$. Therefore, $\xi^{!}[W]^{\text {vir }}=\eta_{2}^{*}\left[\mathbf{D}_{2}\right]$.

It remains to show that $\eta_{1}^{*}\left[\mathbf{D}_{1}\right] \sim_{\text {rat }} \eta_{2}^{*}\left[\mathbf{D}_{2}\right]$. Our strategy is to transform it into a problem about commutativity of Gysin maps and then apply work in [Vi]. Let $\rho_{1}: V_{1} \rightarrow V$ and $\rho_{2}: V \rightarrow V_{2}$ be the embedding and the quotient vector bundle morphisms, and let $1_{V_{1}} \times \rho: V_{1} \rightarrow V_{1} \times_{Z} V$ be the product morphism. Let $\Gamma$ be the graph of the relative Kuranishi map $g \in M \otimes_{A} E_{2}$ and let $0_{V_{i}}$ be the scheme of the 0 -section of $V_{i}$. We set

$$
Y=V_{1} \times_{Z} \Gamma, \quad X_{1}=\left(1_{V_{1}} \times \rho_{1}\right)\left(V_{1}\right) \times_{V_{1} \times_{Z} V} Y, \quad X_{2}=\left(0_{V_{1}} \times_{Z} V\right) \times_{V_{1} \times_{Z} V} Y .
$$

The scheme $Y$ is a subscheme of $V_{1} \times{ }_{Z} V$ and $X_{1}$ and $X_{2}$ are subschemes of $Y$. Clearly, $X_{1} \cong \hat{W} \times_{W} S, X_{2} \cong \Gamma$ and $X_{1} \times_{V_{1} \times_{Z} V} X_{2} \cong \Gamma \times_{V} 0_{V}$. It follows that the normal cone $C_{X_{2} / Y}$ is $V_{1} \times{ }_{Z} \Gamma$, a cone over $X_{2}$. Now let $\mathcal{B}_{1}(S)$ be the normal cone to $C_{X_{2} / Y} \times_{Y} X_{1}$ in $C_{X_{2} / Y}$. Since $C_{X_{2} / Y} \times_{Y} X_{1}$ is $V_{1} \times{ }_{Z}\left(\Gamma \times{ }_{V} 0_{V}\right)$, the scheme $\mathcal{B}_{1}(S)$ is the pull back of $C_{\Gamma \times_{V} 0_{V} / \Gamma} \subset V$ under

$$
\left(V_{1} \times_{Z} V\right) \times_{Z}\left(\hat{W}_{0} \times_{W_{0}} S\right) \stackrel{\mathrm{pr}_{2}}{\longrightarrow} V \times_{Z}\left(\hat{W}_{0} \times_{W_{0}} S\right) .
$$

Let $B_{1}(S)=\mathcal{B}_{1}(S) \times{ }_{Z} S$. Then $B_{1}\left(S_{\alpha}\right)$ patch together to form a cone

$$
\mathbf{B}_{1} \subset \operatorname{Vect}(\mathcal{L}) \times_{W_{0}} \operatorname{Vect}\left(\mathcal{E}_{2}\right) .
$$

The cone $\mathbf{B}_{1}$ is the pull back of $\mathbf{D}_{1} \subset \operatorname{Vect}\left(\mathcal{E}_{2}\right)$ via

$$
\operatorname{Vect}(\mathcal{L}) \times_{W_{0}} \operatorname{Vect}\left(\mathcal{E}_{2}\right) \stackrel{\mathrm{pr}_{2}}{\longrightarrow} \operatorname{Vect}\left(\mathcal{E}_{2}\right) .
$$

Hence if we let $\eta_{0}$ be the zero section of $\operatorname{Vect}(\mathcal{L}) \times{ }_{W_{0}} \operatorname{Vect}\left(\mathcal{E}_{2}\right)$, then $\eta_{0}^{*}\left[\mathbf{B}_{1}\right]=\eta_{1}^{*}\left[\mathbf{D}_{1}\right]$. 
Next, we let $\mathcal{B}_{2}(S)$ be the normal cone to $C_{X_{1} / Y} \times_{Y} X_{2}$ in $C_{X_{1} / Y}$. The cone $\mathcal{B}_{2}(S)$ is canonically a subcone of $\left(V_{1} \times{ }_{Z} V\right) \times_{Z}\left(\hat{W}_{0} \times{ }_{W_{0}} S\right)$. We claim that

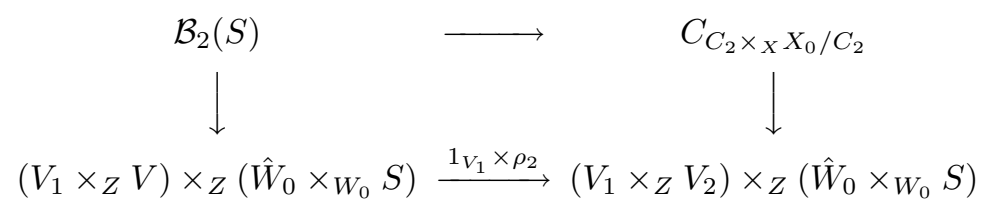

is a fiber square. We first look at $C_{X_{1} / Y}$. Let $h: V_{1} \times_{Z} V \rightarrow V_{1} \times_{Z} V$ be the isomorphism defined by $h(a, b)=\left(a, b-\rho_{1}(a)\right)$. Then $\left.h\right|_{\left(1_{V_{1}} \times \rho_{1}\right)\left(V_{1}\right)}$ is an isomorphism between $\left(1_{V_{1}} \times \rho_{1}\right)\left(V_{1}\right)$ and $V_{1} \times{ }_{Z} 0_{V}$. Further, under $1_{V_{1}} \times \rho_{2}: V_{1} \times{ }_{Z} V \rightarrow V_{1} \times{ }_{Z} V_{2}$, $h(Y)$ is isomorphic to $V_{1} \times Z\left(0_{V_{2}} \times_{V_{2}} \Gamma_{f}\right)$. Therefore, the subcone $C_{X_{1} / Y}$ of

$$
C_{\left(1_{V_{1}} \times \rho_{1}\right)\left(V_{1}\right) / V_{1} \times z V} \times_{V_{1} \times V_{2} V_{2}} X_{1}=V \times{ }_{Z} X_{1}
$$

fits into the Cartesian square

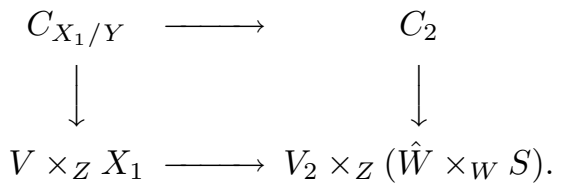

(Note that $X_{1}$ is canonically isomorphic to $\hat{W} \times{ }_{W} S$.) From this description, we immediately see that $C_{X_{1} / Y} \times_{Y} X_{2}$ fits into the Cartesian square

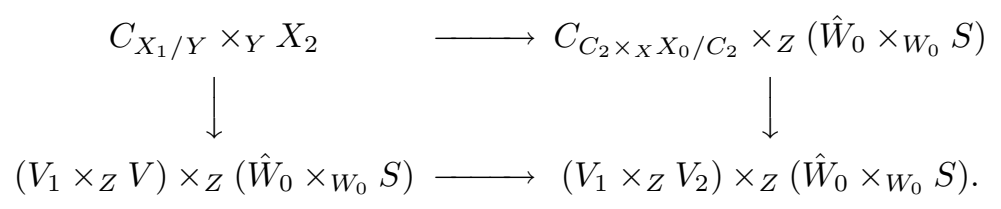

This proves the claim. Finally, we let $B_{2}(S)=\mathcal{B}_{2}(S) \times{ }_{Z} S$. For the same reason, $B_{2}\left(S_{\alpha}\right)$ patch together to form a cone $\mathbf{B}_{2} \subset \operatorname{Vect}(\mathcal{L}) \times_{W_{0}} \operatorname{Vect}\left(\mathcal{E}_{2}\right)$. From the local description, we see that $\left[\mathbf{B}_{2}\right]$ is the pull back of $\left[\mathbf{D}_{2}\right] \in Z_{*}\left(\operatorname{Vect}(\mathcal{L}) \times_{W_{0}} \operatorname{Vect}\left(\mathcal{F}_{2}\right)\right)$. Therefore $\xi^{!}[W]^{\text {vir }}=\eta_{0}^{*}\left[\mathbf{B}_{2}\right]$.

It remains to show that $\left[\mathbf{B}_{1}\right] \sim_{\text {rat }}\left[\mathbf{B}_{2}\right]$. We will apply the basic Lemma in $[\mathrm{Vi}]$ to construct a cycle $[\mathbf{R}] \in Z_{*}\left(\mathbf{A}^{1} \times \operatorname{Vect}(\mathcal{L}) \times_{W_{0}} \operatorname{Vect}\left(\mathcal{E}_{2}\right)\right)$ such that

$$
[\mathbf{R}] \cap\left[\pi_{\mathbf{A}^{1}}^{-1}(0)\right]-[\mathbf{R}] \cap\left[\pi_{\mathbf{A}^{1}}^{-1}(1)\right]=\left[\mathbf{B}_{1}\right]-\left[\mathbf{B}_{2}\right] .
$$

The main conclusion of the basic Lemma in [Vi] is as follows. Let $Y$ be any reduced and equidimensional scheme and let $X_{1}, X_{2} \subset Y$ be closed subschemes. Let $D_{1}$ be the normal cone to $C_{X_{1} / Y} \times_{Y} X_{2}$ in $C_{X_{1} / Y}$ and let $D_{2}$ be the normal cone to $C_{X_{2} / Y} \times_{Y} X_{1}$ in $C_{X_{2} / Y}$, both are canonically embedded in $C_{X_{1} / Y} \times_{Y} C_{X_{2} / Y}$. Then there is a cycle $[R] \in Z_{*}\left(\mathbf{A}^{1} \times C_{X_{1} / Y} \times_{Y} C_{X_{2} / Y}\right)$ such that

$$
[R] \cap\left[\pi_{\mathbf{A}^{1}}^{-1}(0)\right]-[R] \cap\left[\pi_{\mathbf{A}^{1}}^{-1}(1)\right]=\left[D_{1}\right]-\left[D_{2}\right] .
$$

Further, $R$ is canonical under étale base change. The reason that we cannot apply this result directly to our choice of $X_{1}, X_{2} \subset Y$ is that the ambient scheme $Y$ in our situation, which is $V \times_{Z} \Gamma$, may not be equidimensional. However, because of Lemma 3.3, we will argue that the basic Lemma still applies.

We let $S=S_{\alpha}$ be one of the open sets in the covering of $W_{0}$ and let $T_{1}, \cdots, T_{l}$ be the irreducible components of $S$ (with reduced scheme structure). We fix one of these components, denoted by $T . T \subset W_{0}$ is a locally closed affine subscheme. We then form cycles $\mathcal{B}_{1}(T)$ and $\mathcal{B}_{2}(T)$. Because the corresponding $Y$ in constructing 
$\mathcal{B}_{i}(T)$, which is $\left(V \times_{Z} \Gamma\right) \times_{Z}\left(Z \times_{S} T\right)$, is reduced and equidimensional, the basic Lemma provides a cycle

$$
\mathcal{R}(T) \in Z_{*}\left(\mathbf{A}^{1} \times\left(V_{1} \times{ }_{Z} V\right) \times{ }_{Z} T\right)
$$

such that

$$
[\mathcal{R}(T)] \cap\left[\pi_{\mathbf{A}^{1}}^{-1}(0)\right]-[\mathcal{R}(T)] \cap\left[\pi_{\mathbf{A}^{1}}^{-1}(1)\right]=\left[\mathcal{B}_{1}(T)\right]-\left[\mathcal{B}_{2}(T)\right] .
$$

We need to show that the collection $\left\{\mathcal{R}\left(T_{\alpha}\right)\right\}$ provides a global cycle $[\mathbf{R}]$ as required. For this, we need a comparison Lemma similar to Lemma 3.3. Let $q \in S$ be any closed point, let $Z_{q}$ be $Z \times_{S}\{q\}$, let $\hat{Z}$ be the formal completion of $Z$ along $Z_{q}$ and let $\hat{q}$ be the formal completion of $S$ along $q$. Note that $\hat{q}$ is canonically a subscheme of $Z_{q}$. For $i=1,2$ or $\emptyset$, we let $\hat{V}_{i}$ be the formal completion of $V_{i} \times{ }_{Z} \hat{Z}$ along its zero section $0_{V_{i}} \times{ }_{Z} \hat{Z}$.

Sublemma. There is a morphism $\varphi: \hat{Z} \rightarrow Z_{q}$ and there are isomorphisms $\phi_{i}: \hat{V}_{i} \rightarrow$ $\left(\hat{V}_{i} \times{ }_{\hat{Z}} Z_{q}\right) \times_{Z_{q}} \hat{Z}$, where $i=1,2$ and $\emptyset$, for which the following hold.

(1) Let $\iota: \hat{q} \rightarrow \hat{Z}$ be the inclusion induced by $\hat{q} \rightarrow S \rightarrow Z$. Then the restriction of $\varphi$ to $\iota(\hat{q})$ factors through the subscheme $\hat{q} \rightarrow Z_{q}$ and the factored morphism $\iota(\hat{q}) \rightarrow \hat{q}$ is the identity map between $\hat{q}=\iota(\hat{q})$ and $\hat{q} \subset Z_{q}$.

(2) For $i=1,2$ and $\emptyset, \phi_{i}\left(0_{\hat{V}_{i}}\right)=\left(0_{\hat{V}_{i}} \times_{\hat{Z}} Z_{q}\right) \times_{Z_{q}} \hat{Z}$ and the restrictions of $\phi_{i}$ to $\hat{V}_{i} \times \hat{Z} Z_{q}$ are the identity morphisms of $\hat{V}_{i} \times \hat{Z}_{Z} Z_{q}$.

(3) We have the commutative diagram

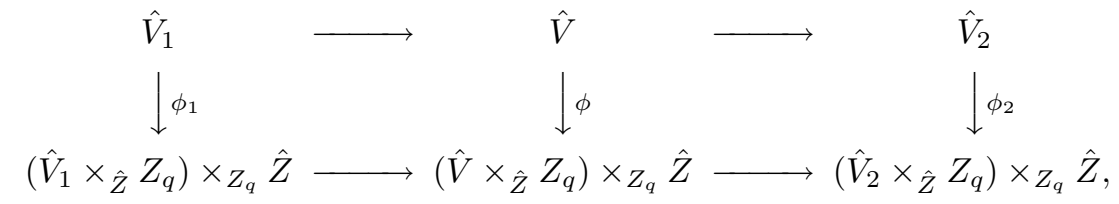

where the lower sequence is induced by $\hat{V}_{1} \stackrel{\hat{\rho}_{1}}{\longrightarrow} \hat{V} \stackrel{\hat{\rho}_{2}}{\longrightarrow} \hat{V}_{2}$.

(4) $\phi\left(\Gamma_{g} \times{ }_{Z} \hat{Z}\right)=\left(\Gamma_{g} \times_{Z} Z_{q}\right) \times_{Z_{q}} \hat{Z}$ and $\phi_{2}\left(\Gamma_{f} \times{ }_{Z} \hat{Z}\right)=\left(\Gamma_{f} \times_{Z} Z_{q}\right) \times_{Z_{q}} \hat{Z}$.

Proof. The proof is similar to the proof of Lemma 3.3. The only modification is to make sure that the morphisms $\rho_{1}$ and $\rho_{2}$ and the schemes $\Gamma_{g}$ and $\Gamma_{f}$ are compatible. This can be done easily following the argument used to construct the relative Kuranishi families of $\mathcal{T}_{W_{0}}^{\bullet}$ from $\mathcal{T}_{W}^{\bullet}$. We will omit the details.

Now if we view $q \in W_{0}$ as an affine subscheme, we obtain the schemes $\mathcal{B}_{1}(q)$ and $\mathcal{B}_{2}(q)$, and the cycle $\mathcal{R}(q)$. Assume that $q \in T_{i}$. Let $\hat{T}_{i}$ be the formal completion of $T_{i}$ along $q$. Then the flat morphism

$$
\begin{aligned}
\left(\hat{V}_{1} \times_{\hat{Z}} \hat{V}\right) \times_{S} \hat{T}_{i} \longrightarrow\left(\hat{V}_{1} \times \hat{Z} \hat{V}\right) \stackrel{\phi_{1} \times \phi}{\longrightarrow}\left(\left(\hat{V}_{1} \times \hat{Z} \hat{V}\right) \times_{\hat{Z}} Z_{q}\right) \times_{Z_{q}} \hat{Z} \\
\stackrel{\mathrm{pr}_{1}}{\longrightarrow} \hat{\left(V_{1} \times \hat{Z}\right.} \times_{\hat{Z}} \hat{V} \times_{\hat{Z}} Z_{q}
\end{aligned}
$$

induces isomorphisms between respective $X_{1}, X_{2}$ and $Y$ in the construction of $\mathcal{B}_{j}\left(\hat{T}_{i}\right)$ and $\mathcal{B}_{j}(q)$. Let

$$
h_{1}: \mathbf{A}^{1} \times\left(V_{1} \times{ }_{Z} V\right) \times{ }_{S} \hat{T}_{i} \rightarrow \mathbf{A}^{1} \times\left(V_{1} \times{ }_{Z} V\right) \times{ }_{Z} Z_{q},
$$

where $h_{1}$ is induced by $\hat{T}_{i} \subset \hat{Z} \stackrel{\varphi}{\longrightarrow} Z_{q}$, and let

$$
h_{2}: \mathbf{A}^{1} \times\left(V_{1} \times_{Z} V\right) \times_{S} \hat{T}_{i} \rightarrow \mathbf{A}^{1} \times\left(V_{1} \times_{Z} V\right) \times_{S} T_{i}
$$


be the obvious morphism. Note that both are flat. We claim that

$$
h_{1}^{*}(\mathcal{R}(q))=h_{2}^{*}\left(\mathcal{R}\left(T_{i}\right)\right) .
$$

This is not exactly what was proved in [Vi], since

$$
H:\left(\hat{V}_{1} \times_{\hat{Z}} \hat{V}\right) \times_{S} \hat{T}_{i} \rightarrow\left(\hat{V}_{1} \times_{\hat{Z}} \hat{V}\right) \times_{S} \hat{Z} \longrightarrow\left(\hat{V}_{1} \times_{\hat{Z}} \hat{V}\right) \times_{\hat{Z}} Z_{q}
$$

is not étale. However, it is clear that $H$ is of the form $\hat{U} \rightarrow U_{1} \times U_{2} \stackrel{\mathrm{pr}_{1}}{\longrightarrow} U_{1}$, where $U_{1}$ and $U_{2}$ are two reduced, irreducible formal complete schemes each supported at a single closed point and $\hat{U}$ is the formal completion of $U_{1} \times U_{2}$ along its closed point. A step by step check of the proof of the basic Lemma in [Vi] shows that the isomorphism (3.4) does hold.

It is clear now how to construct the cycle $\mathcal{R}(S) \in Z_{*}\left(\mathbf{A}^{1} \times\left(V_{1} \times{ }_{Z} V\right) \times{ }_{Z} S\right)$. We let the support of $\mathcal{R}(S)$ be the union of $\operatorname{Supp}\left(\mathcal{R}\left(T_{j}\right)\right)$. Because of the identity (3.4), $\mathcal{R}(S)$ with reduced scheme structure is an equidimensional closed subscheme. Now we assign multiplicity to each irreducible component of $\mathcal{R}(S)$. Let $p \in \mathcal{R}(S)$ be a general point of one of its irreducible components, say $C$. Let $q \in S$ be the closed point under $p$. We assume $q \in T_{j}$. The component $C$ corresponds to a unique irreducible component $C^{\prime}$ in $\mathcal{R}\left(T_{j}\right)$ and a unique irreducible component $C_{0}$ in $\mathcal{R}(q)$, by (3.4). Let $m_{C}$ be the multiplicity of $C_{0}$ in $\mathcal{R}(q)$. Since $T_{j}$ is reduced and irreducible, $m_{C}$ is also the multiplicity of $C^{\prime}$ in $\mathcal{R}\left(T_{j}\right)$. We assign $m_{C}$ to be the multiplicity of $C$ in $\mathcal{R}(S)$. Such an assignment is well-defined.

Remark. The cycle $\mathcal{R}(S)$ has the property that for any closed point $q \in S$, the flat pull back $h_{3}^{*}(\mathcal{R}(S))$ is isomorphic to the flat pull back $h_{1}^{*}(\mathcal{R}(q))$, where

$$
h_{3}:\left(\mathbf{A}^{1} \times \operatorname{Vect}(\mathcal{L}) \times_{W_{0}} \operatorname{Vect}\left(\mathcal{E}_{2}\right)\right) \times_{W_{0}} \hat{q} \longrightarrow \mathbf{A}^{1} \times \operatorname{Vect}(\mathcal{L}) \times_{W_{0}} \operatorname{Vect}\left(\mathcal{E}_{2}\right) .
$$

Of course, if such an $\mathcal{R}(S)$ exists, it is unique. The construction using the relative Kuranishi families is to ensure that $\mathcal{R}(S)$ exists.

Finally, it follows from the étale base change property that $\mathcal{R}\left(S_{\alpha}\right)$ patch together to form a cycle

$$
[\mathbf{R}] \in Z_{*}\left(\mathbf{A}^{1} \times_{k} \operatorname{Vect}(\mathcal{L}) \times_{W_{0}} \operatorname{Vect}\left(\mathcal{E}_{2}\right)\right) .
$$

Because $\mathcal{R}(q)$ provides a rational equivalence of $\left[\mathcal{B}_{1}(q)\right]$ and $\left[\mathcal{B}_{2}(q)\right]$, $\mathbf{R}$ provides a rational equivalence of $\left[\mathbf{B}_{1}\right]$ and $\left[\mathbf{B}_{2}\right]$. Therefore, $\xi^{*}[W]^{\mathrm{vir}}=\left[W_{0}\right]^{\mathrm{vir}}$. This completes the proof of the proposition.

\section{Gromov-Witten invariants of SMooth VARIETIES}

Let $X$ be a smooth projective variety, $n, g$ integers, and $\alpha \in A_{1} X / \sim_{\text {alg. }}$. The $\mathrm{GW}$-invariants are defined by taking intersections on the moduli space of stable maps from $n$-pointed genus $g$ curves to $X$ such that their image cycles are in $\alpha$. We denote this moduli space by $\mathcal{M}_{\alpha, g, n}^{X}$. When $\mathcal{M}_{\alpha, g, n}^{X}$ has the expected dimension, then the GW-invariants can be defined as usual. However, this rarely happens. Thus we need to use the virtual moduli cycles to define these invariants.

Let $S$ be an affine scheme and let $\eta \in \mathfrak{F}_{\alpha, g, n}^{X}(S)$ be an element represented by the morphism $f: \mathcal{X} \rightarrow X$, where $\mathcal{X}$ is a curve over $S$ with marked sections $D \subset \mathcal{X}$ understood. Then the standard choice of the tangent-obstruction complex of $\mathfrak{F}_{\alpha, g, n}^{X}$ is

$$
\mathcal{T}^{\bullet} \mathfrak{F}_{\alpha, g, n}^{X}(\eta)(\mathcal{F})=\left[\mathcal{E} x t_{\mathcal{X} / S}^{\bullet}\left(\left[f^{*} \Omega_{X} \rightarrow \Omega_{\mathcal{X} / S}(D)\right], \pi_{S}^{*} \mathcal{F}\right)\right]
$$


where $\mathcal{F} \in \mathfrak{M o d}_{S}$ and $\pi_{S}: \mathcal{X} \rightarrow S$ is the projection. We now show that there are complexes of locally free sheaves over $\mathfrak{F}_{\alpha, g, n}^{X}$ so that their sheaf cohomologies are $\mathcal{T} \bullet \mathfrak{F}_{\alpha, g, n}^{X}$.

We fix a sufficiently ample invertible sheaf $\mathcal{L}$ on $X$ and then form the exact sequence

$$
0 \longrightarrow \mathcal{W}_{2} \longrightarrow \mathcal{W}_{1} \longrightarrow f^{*} \Omega_{X} \longrightarrow 0
$$

where $\mathcal{W}_{1} \rightarrow f_{S}^{*} \Omega_{X}$ is the natural surjective homomorphism

$$
\pi_{S}^{*} \pi_{S *}\left(\omega_{\mathcal{X} / S}(D)^{\otimes 5} \otimes f^{*}\left(\mathcal{L} \otimes \Omega_{X}\right)\right) \otimes\left(\omega_{\mathcal{X} / S}(D)^{\otimes 5} \otimes f^{*} \mathcal{L}\right)^{-1} \longrightarrow f^{*} \Omega_{X} .
$$

We then form complexes

$$
\mathcal{A}_{\eta}^{\bullet}=\left[\mathcal{W}_{2} \rightarrow 0\right] \quad \text { and } \quad \mathcal{B}_{\eta}^{\bullet}=\left[\mathcal{W}_{1} \rightarrow \Omega_{\mathcal{X} / S}(D)\right]
$$

indexed at -1 and 0 with $\mathcal{W}_{1} \rightarrow \Omega_{\mathcal{X} / S}(D)$ the composite $\mathcal{W}_{1} \rightarrow f^{*} \Omega_{X} \rightarrow \Omega_{\mathcal{X} / S}(D)$. Let $\mathcal{C}_{\eta}^{\bullet}=\left[f^{*} \Omega_{X} \rightarrow \Omega_{\mathcal{X} / S}(D)\right]$. Then we have an exact sequence of complexes

$$
0 \longrightarrow \mathcal{A}_{\eta}^{\bullet} \longrightarrow \mathcal{B}_{\eta}^{\bullet} \longrightarrow \mathcal{C}_{\eta}^{\bullet} \longrightarrow 0,
$$

and hence a long exact sequence of sheaf cohomologies

$$
\mathcal{E} t_{\mathcal{X} / S}^{1}\left(\mathcal{C}_{\eta}^{\bullet}, \mathcal{O}_{\mathcal{X}}\right) \rightarrow \mathcal{E} x t_{\mathcal{X} / S}^{1}\left(\mathcal{B}_{\eta}^{\bullet}, \mathcal{O}_{\mathcal{X}}\right) \rightarrow \mathcal{E} x t_{\mathcal{X} / S}^{1}\left(\mathcal{A}_{\eta}^{\bullet}, \mathcal{O}_{\mathcal{X}}\right) \rightarrow \mathcal{E} x t_{\mathcal{X} / S}^{2}\left(\mathcal{C}_{\eta}^{\bullet}, \mathcal{O}_{\mathcal{X}}\right) .
$$

Since $\mathcal{L}$ is sufficiently ample, $\mathcal{E} x t_{\mathcal{X} / S}^{i}\left(\mathcal{B}_{\eta}^{\bullet}, \mathcal{O}_{\mathcal{X}}\right)$ and $\mathcal{E} x t_{\mathcal{X} / S}^{i}\left(\mathcal{A}_{\eta}^{\bullet}, \mathcal{O}_{\mathcal{X}}\right)$ vanish for $i \neq 1$. Hence

$$
\mathcal{E}_{\eta, 1}=\mathcal{E}_{x} t_{\mathcal{X} / S}^{1}\left(\mathcal{B}_{\eta}^{\bullet}, \mathcal{O}_{\mathcal{X}}\right) \quad \text { and } \quad \mathcal{E}_{\eta, 2}=\mathcal{E} t_{\mathcal{X} / S}^{1}\left(\mathcal{A}_{\eta}^{\bullet}, \mathcal{O}_{\mathcal{X}}\right)
$$

are locally free and the sheaf cohomology of $\mathcal{E}_{\eta}^{\bullet}=\left[\mathcal{E}_{\eta, 1} \rightarrow \mathcal{E}_{\eta, 2}\right]$ is $\mathcal{T} \bullet \mathfrak{F}_{\alpha, g, n}^{X}(\eta)$. It is straightforward to check that the collection $\left\{\mathcal{E}_{\eta}^{\bullet}\right\}$ satisfies the base change property in Definition 1.1, hence it forms a complex of sheaves over $\mathfrak{F}_{\alpha, g, n}^{X}$.

To construct the virtual moduli cycle $\left[\mathcal{M}_{\alpha, g, n}^{X}\right]^{\text {vir }}$, we need to address one technical issue, namely, $\mathcal{M}_{\alpha, g, n}^{X}$ does not admit universal families due to the presence of non-trivial automorphisms. An automorphism of a morphism $f$ from $C$ to $X$ is an automorphism $\varphi: C \rightarrow C$ fixing its marked points such that $\varphi \circ f=\varphi$. Because $f$ is stable, $\operatorname{Aut}(f)$ is finite. There are two approaches to get around this difficulty. One is to realize the moduli space as a quotient by a reductive group, say $G$. The other is to use the intersection theory on stacks developed in [Vi]. The former relies on constructing $G$-equivariant data and then descending them to the quotient space. This can be done directly if the quotient is a good quotient. Otherwise, the étale slice of the group action can be used to study the descent problem. This approach allows one to work with Fulton's operational cohomology theory of $\mathcal{M}_{\alpha, g, n}^{X}$, rather than the parallel theory on the moduli stack of $\mathfrak{F}_{\alpha, g, n}^{X}$.

From [Al], there is a quasi-projective scheme $\mathcal{Q}$ and a reductive group $G$ acting on $\mathcal{Q}$ such that $\mathcal{M}_{\alpha, g, n}^{X}$ is the categorical quotient of $\mathcal{Q}$ by $G$. Over $\mathcal{Q}$, there is a universal family

$$
\{F: D \subset \mathcal{X} \longrightarrow X\}=\xi \in \mathfrak{F}_{\alpha, g, n}^{X}(\mathcal{Q})
$$

acted on by $G$. For any closed point $w \in \mathcal{Q}$, the stabilizer $G_{w} \subset G$ of $w$ is naturally the automorphism group of $F_{w}: D_{w} \subset \mathcal{X}_{w} \rightarrow X$. Now by using this family we can construct the complex $\mathcal{E}^{\bullet}:=\mathcal{E}_{\xi}^{\bullet}$ in (4.1), after fixing a very ample invertible sheaf $\mathcal{L}$. By our construction, $\mathcal{T} \bullet \mathfrak{F}_{\alpha, g, n}^{X}(\xi)=\mathfrak{h}^{\bullet}\left(\mathcal{E}^{\bullet}\right)$. Further, both $\mathcal{E}^{\bullet}$ and $\mathcal{T} \bullet \mathfrak{F}_{\alpha, g, n}^{X}(\xi)$ are canonically $G$-linearized and the identity is $G$-equivariant. 
We now construct the virtual cycle $\left[\mathcal{M}_{\alpha, g, n}^{X}\right]^{\text {vir }}$ with the complex $\mathcal{E}^{\bullet}$ provided. Let $z \in \mathcal{M}_{\alpha, g, n}^{X}$ be any closed point and $w \in \pi^{-1}(z)$, where $\pi: \mathcal{Q} \rightarrow \mathcal{M}_{\alpha, g, n}^{X}$ is the quotient projection. Let $G_{w}$ be the stabilizer of $w$. By combining an argument in [Ko2] and the construction of [Al], we can find a $G_{w}$-invariant slice $S \subset \mathcal{Q}$ containing $w$ such that $S / G_{w}$ is an étale neighborhood of $z \in \mathcal{M}_{\alpha, g, n}^{X}$. Let $\xi_{S} \in$ $\mathfrak{F}_{\alpha, g, n}^{X}(S)$ be the object associated to the restriction of $F: \mathcal{X} \rightarrow X$ to fibers over $S$. Clearly, $\mathcal{T} \bullet \mathfrak{F}_{\alpha, g, n}^{X}\left(\xi_{S}\right)$ is a tangent-obstruction complex of $S$ and $\mathcal{E}_{S}^{\bullet}=\mathcal{E}^{\bullet} \otimes_{\mathcal{O}_{\mathcal{Q}}} \mathcal{O}_{S}$ is the complex whose sheaf cohomology is $\mathcal{T}^{\bullet} \mathfrak{F}_{\alpha, g, n}^{X}\left(\xi_{S}\right)$. Therefore, by applying the construction in the previous sections, we obtain a canonical cone

$$
C^{\mathcal{E}_{S}^{\bullet}} \subset \operatorname{Vect}\left(\mathcal{E}_{S, 2}\right)=\operatorname{Vect}\left(\mathcal{E}_{2}\right) \times_{\mathcal{Q}} S .
$$

Let $C^{\mathcal{E}_{S}^{\bullet}} / G_{w} \subset \operatorname{Vect}\left(\mathcal{E}_{2}\right) \times_{\mathcal{Q}} S / G_{w}$ be their quotients. Assume that $T$ is another $G_{w}$-invariant slice passing through $w^{\prime} \in O_{w}$ such that $T / G_{w}$ is an étale neighborhood of $z$; then we obtain the cones $C^{\mathcal{E}_{T}^{\bullet}} \subset \operatorname{Vect}\left(\mathcal{E}_{2}\right) \times{ }_{\mathcal{Q}} T$ and their quotients by $G_{w}$. Since $\mathcal{E}^{\bullet}$ is $G$-linearized, by Lemma 3.2, the pull-back of these schemes to $S / G_{w} \times_{\mathcal{M}_{\alpha, g, n}^{X}} T / G_{w}$ from $S / G_{w}$ and $T / G_{w}$ are naturally isomorphic. Hence the collection $\operatorname{Vect}\left(\mathcal{E}_{2}\right) \times{ }_{\mathcal{Q}} S / G_{w}$ descends to a scheme $\operatorname{Vect} \mathcal{M}_{\mathcal{M}}\left(\mathcal{E}_{2}\right)$ over $\mathcal{M}_{\alpha, g, n}^{X}$, and the collection $C^{\mathcal{E}_{S}^{\bullet}} / G_{w}$ descends to a scheme $C_{\mathcal{M}}^{\mathcal{E}^{\bullet}}$ which is a subscheme of $\operatorname{Vect}_{\mathcal{M}}\left(\mathcal{E}_{2}\right)$. Note that $\operatorname{Vect}_{\mathcal{M}}\left(\mathcal{E}_{2}\right)$ is not necessarily a vector bundle. We will call $\operatorname{Vect}_{\mathcal{M}}\left(\mathcal{E}_{2}\right)$ the $\mathbf{Q}$-descent of the vector bundle $\operatorname{Vect}\left(\mathcal{E}_{2}\right)$.

Similar to the ordinary case, we define the virtual moduli cycle $\left[\mathcal{M}_{\alpha, g, n}^{X}\right]^{\text {vir }}$ to be

$$
s^{*}\left[C_{\mathcal{M}}^{\mathcal{E}^{\bullet}}\right] \in A_{*}\left(\mathcal{M}_{\alpha, g, n}^{X}\right) \otimes_{\mathbf{z}} \mathbf{Q},
$$

where $s: \mathcal{M}_{\alpha, g, n}^{X} \rightarrow \operatorname{Vect}_{\mathcal{M}}\left(\mathcal{E}_{2}\right)$ is the zero section and $s^{*}$ is the Gysin map. Note that $s^{*}$ is well-defined. One way of seeing it is by using the description of Gysin maps in terms of Chern classes [Fu, §6.1]. This way, to define $s^{*}$, it suffices to define the Chern classes of $\mathbf{Q}$-descents of vector bundles (i.e. V-vector bundles), which are known to exist with rational coefficients.

Lemma 4.1. The cycle $\left[\mathcal{M}_{\alpha, g, n}^{X}\right]^{\text {vir }}$ is independent of the choice of the complex $\mathcal{E}^{\bullet}$ making $\mathcal{T} \bullet \mathfrak{F}_{\alpha, g, n}^{X}(\xi)=\mathfrak{h} \bullet(\mathcal{E} \bullet)$.

Proof. We will apply Corollary 3.5 to prove the invariance. Clearly, by the proof of Corollary 3.5 and the above construction, it suffices to show that if $\mathcal{F}^{\bullet}=\left[\mathcal{F}_{1} \rightarrow \mathcal{F}_{2}\right]$ is another complex of $G$-linearized locally free sheaves such that $\mathcal{T} \bullet \mathfrak{F}_{\alpha, g, n}^{X}(\xi)=$ $\mathfrak{h}^{\bullet}\left(\mathcal{F}^{\bullet}\right)$ and that the identity is $G$-equivariant, then there is a $G$-linearized locally free sheaf of $\mathcal{O}_{\mathcal{Q}}$-modules $\mathcal{K}$ and surjective $G$-linearized sheaf homomorphisms $\mathcal{K} \rightarrow$ $\mathcal{E}_{2}$ and $\mathcal{K} \rightarrow \mathcal{F}_{2}$ such that

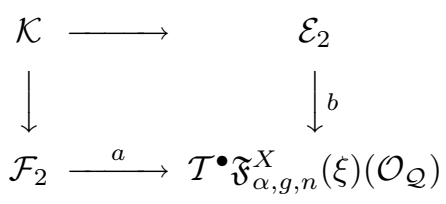

is commutative. We first let $\mathcal{K}_{0}$ be the pull-back of $(a, b)$, where $a$ and $b$ are shown in the above square. Then $\mathcal{K}_{0}$ is canonically $G$-linearized. It remains to find a $G$-linearized locally free sheaf $\mathcal{K}$ so that $\mathcal{K}_{0}$ is a $G$-quotient sheaf of $\mathcal{K}$. Let $L$ be an ample $G$-linearized line bundle on $\mathcal{Q}$. Such an $L$ exists following [Al]. Let $w \in \mathcal{Q}$ be any closed point and $O_{w}$ its $G$-orbit, which is closed. Using locally free sheaves 
$\mathcal{E}_{2}$ and $\mathcal{F}_{2}$, we can find a $G$-equivariant surjective homomorphism

$$
\eta_{w}: \mathcal{E}_{2} \oplus \mathcal{F}_{2} \longrightarrow \mathcal{K}_{0} \otimes \mathcal{O}_{O_{w}} .
$$

Since $G_{w}$ is finite, for some power $L^{\otimes n_{w}}$, the $G_{w}$-action on $L^{\otimes n_{w}} \otimes k(w)$ is trivial. Because $\mathcal{Q}$ is quasi-projective and $L$ is ample, for some large $m$, the homomorphism

$$
\eta_{w}^{\prime}:\left(\mathcal{E}_{2} \oplus \mathcal{F}_{2}\right) \otimes \mathcal{O}\left(L^{n_{w} m}\right) \longrightarrow \mathcal{K}_{0} \otimes \mathcal{O}_{O_{w}}
$$

induced by $\eta_{w}$, which is still surjective and $G$-equivariant, lifts to a global homomorphism

$$
\eta:\left(\mathcal{E}_{2} \oplus \mathcal{F}_{2}\right) \otimes \mathcal{O}\left(L^{n_{w} m}\right) \longrightarrow \mathcal{K}_{0}
$$

Then applying the Reynolds operator, we can assume that $\eta$ is also $G$-equivariant and its restriction to $O_{w}$ is $\eta_{w}^{\prime}$. Since $\mathcal{Q}$ is quasi-projective, a finite sum of sheaves of this type gives us the desired $G$-equivariant surjective homomorphism $\mathcal{K} \rightarrow \mathcal{K}_{0}$. Therefore, by Corollary 3.5, if we let $\operatorname{Vect}_{\mathcal{M}}(\mathcal{K})$ be the $\mathbf{Q}$-descent of the vector bundle $\operatorname{Vect}(\mathcal{K})$ and let $\phi_{1}: \operatorname{Vect}_{\mathcal{M}}(\mathcal{K}) \rightarrow \operatorname{Vect}_{\mathcal{M}}\left(\mathcal{E}_{2}\right)$ and $\phi_{2}: \operatorname{Vect}_{\mathcal{M}}(\mathcal{K}) \rightarrow \operatorname{Vect}_{\mathcal{M}}\left(\mathcal{F}_{2}\right)$ be the induced morphisms between the $V$-vector bundles, then $\phi_{1}^{*}\left[C_{\mathcal{M}}^{\mathcal{E}^{\bullet}}\right]=\phi_{2}^{*}\left[C_{\mathcal{M}}^{\mathcal{F}^{\bullet}}\right]$. Therefore, $\left[\mathcal{M}_{\alpha, g, n}^{X}\right]^{\text {vir }}$ is independent of the choice of the complex $\mathcal{E}^{\bullet}$.

In the remainder of this paper, we will define the GW-invariants of any smooth projective variety and prove some of its basic properties. From now on, unless otherwise mentioned we will only consider homology theory with rational coefficients. We will denote the (operational) cohomology and homology by $A^{*}$ and $A_{*}$ respectively. When the varieties are over complex numbers, sometimes we will use the singular homology theory, which we will denote by $H_{*}$. We now give the definition of GW-invariants of any smooth projective variety $X$. We fix the $\alpha \in A_{1} X / \sim_{\text {alg }}$ and the integers $g$ and $n$ as before so that $2 g+n \geq 3$. Let

$$
\left[\mathcal{M}_{\alpha, g, n}^{X}\right]^{\mathrm{vir}} \in A_{*}\left(\mathcal{M}_{\alpha, g, n}^{X}\right)
$$

be the virtual moduli cycle. By using the Riemann-Roch theorem, it is a purely $(3-\operatorname{dim} X)(g-1)+n+\alpha \cdot c_{1}(X)$ dimensional cycle. Let $\pi_{n}^{\alpha}: \mathcal{M}_{\alpha, g, n}^{X} \rightarrow \mathcal{M}_{g, n}$ be the stable contraction morphism. The $k$-th marked points in curves naturally induce an evaluation morphism $e_{k}: \mathcal{M}_{\alpha, g, n}^{X} \rightarrow X$. We let $e v: \mathcal{M}_{\alpha, g, n}^{X} \rightarrow X^{n}$ be the product $e_{1} \times \cdots \times e_{n}$. Paired with the cycle $\left[\mathcal{M}_{\alpha, g, n}^{X}\right]^{\text {vir }}$, we obtain a homomorphism

defined by

$$
\Psi_{\alpha, g, n}^{X}: A^{*}(X)^{\times n} \times A^{*}\left(\mathcal{M}_{g, n}\right) \longrightarrow A_{*}\left(\mathcal{M}_{\alpha, g, n}^{X}\right)
$$

$$
\Psi_{\alpha, g, n}^{X}(\beta, \gamma)=\left(e v^{*}(\beta) \cup\left(\pi_{n}^{\alpha}\right)^{*}(\gamma)\right)\left(\left[\mathcal{M}_{\alpha, g, n}^{X}\right]^{\mathrm{vir}}\right) .
$$

Composing $\Psi_{\alpha, g, n}^{X}$ with the degree map $A_{*}\left(\mathcal{M}_{\alpha, g, n}^{X}\right) \rightarrow A_{0}\left(\mathcal{M}_{\alpha, g, n}^{X}\right) \rightarrow \mathbf{Q}$, we obtain the GW-invariants

$$
\psi_{\alpha, g, n}^{X}: A^{*}(X)^{\times n} \times A^{*}\left(\mathcal{M}_{g, n}\right) \longrightarrow \mathbf{Q} .
$$

If we fix a polarization $H$ of $X$ and an integer $d$, we can define the GW-invariants

$$
\psi_{d, g, n}^{X}: A^{*}(X)^{\times n} \times A^{*}\left(\mathcal{M}_{g, n}\right) \longrightarrow \mathbf{Q}
$$

as follows. We let $\mathfrak{F}_{d, g, n}^{X}: \mathcal{S} \rightarrow(\text { sets })^{0}$ be the moduli functor of stable morphisms defined similar to $\mathfrak{F}_{\alpha, g, n}^{X}$ except that the condition $f_{*}([C]) \in \alpha$ is replaced by the condition that the degree of $c_{1}(H)\left(f_{*}([C])\right)$ is $d$. Because of $[\mathrm{Al}], \mathfrak{F}_{d, g, n}^{X}$ is coarsely represented by a projective scheme, denoted $\mathcal{M}_{d, g, n}^{X}$. The previous construction 
works for this moduli functor without any change. Consequently we have the virtual moduli cycle $\left[\mathcal{M}_{d, g, n}^{X}\right]^{\text {vir }}$ which in turn defines the GW-invariants $\psi_{d, g, n}^{X}$. When $X$ is a smooth complex projective variety, then we can use the ordinary homology theory to define

$$
\psi_{\alpha, g, n}^{X}: H^{*}(X)^{\times n} \times H^{*}\left(\mathcal{M}_{g, n}\right) \longrightarrow \mathbf{Q},
$$

where $\alpha \in H_{2}(X, \mathbf{Z})$, by using the image of $\left[\mathcal{M}_{\alpha, g, n}^{X}\right]^{\text {vir }}$ in $H_{*}\left(\mathcal{M}_{\alpha, g, n}^{X}\right)$. Here since $\alpha \in H_{2}(X, \mathbf{Z})$, the moduli functor $\mathfrak{F}_{\alpha, g, n}^{X}$ parameterizes all stable morphisms $f$ : $C \rightarrow X$ with $f_{*}([C])=\alpha$ understood as an identity in the singular homology $H_{2}(X, \mathbf{Z})$. Note that if $\alpha \notin H_{2}(X, \mathbf{Z}) \cap H^{0,1}(X, \mathbf{C})^{\perp}$, then $\psi_{\alpha, g, n}^{X} \equiv 0$.

The GW-invariants satisfy some basic properties. One of them is the invariance under deformations of $X$. Let $\pi: X_{T} \rightarrow T$ be a smooth family of relatively projective varieties over $T$. For $t \in T$ we let $X_{t}=\pi^{-1}(t)$ and for $\beta \in A^{*}\left(X_{T}\right)^{\times n}$ we let $\beta_{t} \in A^{*}\left(X_{t}\right)^{\times n}$ be the pull back of $\beta$ under $X_{t} \rightarrow X_{T}$. We fix a relatively ample line bundle of $X_{T} / T$.

Theorem 4.2. Let $X_{T} / T$ be as before with $T$ an irreducible smooth curve. Then for any $d \in \mathbf{Z}$ and cohomology classes $\beta \in A^{*}\left(X_{T}\right)^{\times n}$ and $\gamma \in A^{*}\left(\mathcal{M}_{g, n}\right)$, the values of the $G W$-invariants $\phi_{d, g, n}^{X}\left(\beta_{t}, \gamma\right)$ are independent of $t \in T$.

When $X_{T} / T$ is defined over $\mathbf{C}$, we can use knowledge of $H_{2}\left(X_{t}, \mathbf{Z}\right)$ to prove a finer version of the invariance theorem. Consider the analytic curve

$$
\Lambda:=R_{2} \pi_{*} \mathbf{Z}_{X_{T}} \otimes_{T} R^{k_{1}} \pi_{*} \mathbf{Z}_{X_{T}} \times_{T} \cdots \times_{T} R^{k_{n}} \pi_{*} \mathbf{Z}_{X_{T}}
$$

over $T$. Clearly any point $w \in \Lambda$ corresponds to $w=\left(\alpha_{w}, \beta_{w}\right) \in H_{2}\left(X_{t}, \mathbf{Z}\right) \times$ $H^{*}\left(X_{t}, \mathbf{Z}\right)^{\times n}$ for some $t \in T$. Hence for any $\gamma \in H^{*}\left(\mathcal{M}_{g, n}\right)$ we can define

$$
\Psi_{\gamma}: \Lambda \longrightarrow \mathbf{Q}
$$

that assigns $w$ to $\psi_{\alpha_{w}, g, n}^{X_{t}}\left(\beta_{w} ; \gamma\right)$.

Theorem 4.2' ${ }^{\prime}$ Let $X_{T}$ be defined over $\mathbf{C}$ as before. Assume $T$ is a smooth connected curve. Then for any $\gamma \in H^{*}\left(\mathcal{M}_{g, n}\right)$ the function $\Phi_{\gamma}: \Lambda \rightarrow \mathbf{Q}$ is locally constant.

Proof of Theorem 4.2. We first form the relative moduli functor. For simplicity, we assume $T$ is affine. Let $\mathfrak{S c h}_{T}$ be the category of $T$-schemes and let $\mathfrak{F}_{d, g, n}^{X_{T} / T}$ : $\mathfrak{S c h}_{T} \rightarrow(\text { sets })^{0}$ be the functor that sends any $S \in \mathfrak{S c h}_{T}$ to the subset of $\mathfrak{F}_{d, g, n}^{X_{T}, n}(S)$ consisting of the isomorphism classes of $f: \mathcal{X} \rightarrow X_{T}$ such that

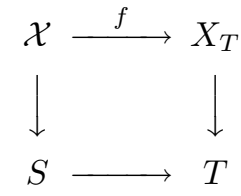

is commutative. $\mathfrak{F}_{d, g, n}^{X_{T} / T}$ is coarsely represented by a $T$-projective scheme $\mathcal{M}_{d, g, n}^{X_{T} / T}$ [Al]. Let $\mathcal{M}_{d, g, n}^{X_{T} / T} \rightarrow T$ be the obvious morphism. Then we have the Cartesian square

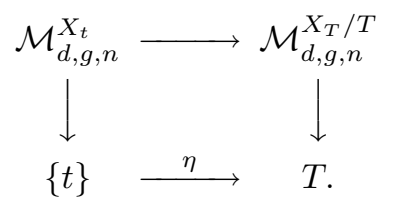


Following the principle of conservation of number [Fu, section 10.2], to prove the theorem it suffices to show that

$$
\eta^{!}\left[\mathcal{M}_{d, g, n}^{X_{T} / T}\right]^{\mathrm{vir}}=\left[\mathcal{M}_{d, g, n}^{X_{t}}\right]^{\mathrm{vir}} .
$$

We first determine the tangent-obstruction complex of $\mathfrak{F}_{d, g, n}^{X_{T} / T}$. Let $S \stackrel{c}{\rightarrow} T$ be any affine scheme over $T$ and $\xi \in \mathfrak{F}_{d, g, n}^{X_{T} / T}(S)$ be represented by $f: \mathcal{X} \rightarrow X$ with marked points $D \subset \mathcal{X}$ understood. From the discussion in section 1 , the tangent of $\mathfrak{F}_{d, g, n}^{X_{T} / T}$ at $\xi$ is

$$
\mathcal{T}^{1} \mathfrak{F}_{d, g, n}^{X_{T} / T}(\xi)(\mathcal{N})=\mathcal{E} x t_{\mathcal{X} / S}^{1}\left(\mathcal{B}_{\xi}^{\bullet}, \mathcal{O}_{\mathcal{X}} \otimes_{\mathcal{O}_{S}} \mathcal{N}\right)
$$

where $\mathcal{N} \in \mathfrak{M o d}_{S}$ and $\mathcal{B}_{\xi}^{\bullet}=\left[f^{*} \Omega_{X_{T}} \rightarrow \Omega_{\mathcal{X} / S}(D)\right]$. We claim that the obstruction to deformations of $f$ lies in the kernel of

$$
\mathcal{E} x t_{\mathcal{X} / S}^{2}\left(\mathcal{B}_{\xi}^{\bullet}, \mathcal{O}_{\mathcal{X}} \otimes_{\mathcal{O}_{S}} \mathcal{N}\right) \stackrel{\beta}{\longrightarrow} \mathcal{E} x t_{\mathcal{X} / S}^{2}\left(\mathcal{C}_{\xi}^{\bullet}, \mathcal{O}_{\mathcal{X}} \otimes_{\mathcal{O}_{S}} \mathcal{N}\right)
$$

where $\mathcal{C}_{\xi}^{\bullet}=\left[f^{*} \pi^{*} \Omega_{T} \rightarrow 0\right]$ and $\pi: X_{T} \rightarrow T$. Here the homomorphism $\beta$ is part of the long exact sequence of cohomologies induced from the short exact sequence

$$
0 \longrightarrow \mathcal{C}_{\xi}^{\bullet} \longrightarrow \mathcal{B}_{\xi}^{\bullet} \longrightarrow \mathcal{A}_{\xi}^{\bullet} \longrightarrow 0
$$

induced by $\pi^{*} \Omega_{T} \rightarrow \Omega_{X_{T}} \rightarrow \Omega_{X_{T} / T}$. Here $\mathcal{A}_{\xi}^{\bullet}$ is the complex $\left[f^{*} \Omega_{X_{T} / T} \rightarrow\right.$ $\left.\Omega_{\mathcal{X} / S}(D)\right]$. Indeed, let $S \rightarrow Y_{0} \rightarrow Y$ be a tuple of $S$-schemes described in Definition 1.2 with $\mathcal{I}_{Y_{0} \subset Y} \cong \mathcal{N}$ and let $f_{0}: \mathcal{X}_{0} \rightarrow X_{t}$ with marked points $D_{0} \subset \mathcal{X}_{0}$ be a family of stable morphisms over $Y_{0}$. Let $D \subset \mathcal{X}$ be an extension of $D_{0} \subset \mathcal{X}_{0}$ and let $o$ be the obstruction to extending $f_{0}$ to $f$ over $Y$. Then using the description of $o$ in section 1 we see immediately that $\beta(o)$ is the image of

$$
\left\{\pi^{*} \Omega_{T} \rightarrow \Omega_{\mathcal{X}_{0} / S}\right\}=0 \in \operatorname{Hom}\left(\pi^{*} \Omega_{T}, \Omega_{\mathcal{X} / S}\right)
$$

in $\operatorname{Ext}_{\mathcal{X}}^{2}\left(\mathcal{C}_{\xi}^{\bullet}, \pi_{S}^{*} \mathcal{N}\right)$, which is zero. We denote the kernel of $\beta$ by $\mathcal{T}^{2} \mathfrak{F}_{d, g, n}^{X_{T} / T}(\xi)(\mathcal{N})$. $\mathcal{T} \bullet \mathfrak{F}_{d, g, n}^{X_{T} / T}$ is the tangent-obstruction complex of $\mathfrak{F}_{d, g, n}^{X_{T} / T}$. Now assume $S \stackrel{c}{\rightarrow} T$ factors through $\{t\} \subset T$. Then for $\mathcal{N} \in \mathfrak{M o d}_{S}$ we have the exact sequence

$$
\begin{aligned}
0 \longrightarrow \mathcal{T}^{1} \mathfrak{F}_{d, g, n}^{X_{t}}(\xi)(\mathcal{N}) & \longrightarrow \mathcal{T}^{1} \mathfrak{F}_{, g, n}^{X_{T} / T}(\xi)(\mathcal{N}) \longrightarrow \mathcal{N} \longrightarrow \\
& \longrightarrow \mathcal{T}^{2} \mathfrak{F}_{d, g, n}^{X_{t}}(\xi)(\mathcal{N}) \longrightarrow \mathcal{T}^{2} \mathfrak{F}_{d, g, n}^{X_{T} / T}(\xi)(\mathcal{N}) \longrightarrow 0
\end{aligned}
$$

Also, from the description of the obstruction classes in section 1 , we see immediately that the obstruction classes are compatible in the sense of Definition 3.8.

To prove the theorem, we need to choose complexes so that they satisfy the technical condition of Proposition 3.9. We fix a sufficiently ample invertible sheaf $\mathcal{L}$ on $X_{t}$. We first let $\mathcal{H}^{\bullet}$ (resp. $\mathcal{K}^{\bullet}$ ) be the complex constructed in (4.1) with $f^{*} \Omega_{X}$ replaced by $f^{*} \Omega_{X_{t}}$ (resp. $f^{*} \Omega_{X_{T}}$ ). We let $\mathcal{G}^{\bullet}$ be the complex in (4.1) with $f^{*} \Omega_{X}$ replaced by $f^{*} \mathcal{O}_{X_{t}}$ and with $\Omega_{\mathcal{X}}(D)$ replaced by 0 . Clear, they fit into the exact sequence $0 \rightarrow \mathcal{H}^{\bullet} \rightarrow \mathcal{K}^{\bullet} \rightarrow \mathcal{G}^{\bullet} \rightarrow 0$. Now let $\mathcal{E}^{\bullet}=\mathcal{H}^{\bullet} \oplus\left[\mathcal{O}_{S} \stackrel{\text { id }}{\rightarrow} \mathcal{O}_{S}\right]$ and let $\mathcal{F}^{\bullet}$ be the kernel of $\mathcal{K}^{\bullet} \rightarrow \mathcal{G}^{\bullet}$. Note that $\mathfrak{h}^{1}\left(\mathcal{G}^{\bullet}\right)=\mathcal{O}_{S}$. We pick a homomorphism $\mathcal{O}_{S} \rightarrow \mathcal{F}_{1}$ so that $\mathcal{O}_{S} \rightarrow \mathcal{F}_{1} \rightarrow \mathfrak{h}^{1}\left(\mathcal{G}^{\bullet}\right)$ is the identity homomorphism. The long exact sequence of the sheaf cohomologies of the resulting exact sequence

$$
0 \longrightarrow\left[0 \rightarrow \mathcal{O}_{S}\right] \longrightarrow \mathcal{E}^{\bullet} \longrightarrow \mathcal{F}^{\bullet} \longrightarrow 0
$$

is exactly the sequence (4.2), and hence the tangent-obstruction complex of $\mathfrak{F}_{d, g, n}^{X_{T} / T}$ satisfies the technical condition of Proposition 3.9. 
Lastly, we need to modify the proof of Proposition 3.9 to accommodate the fact that $\mathcal{M}_{d, g, n}^{X_{T} / T}$ has no universal family, as we did in constructing the virtual moduli cycle. We will omit the details here since it is a repetition of the previous argument. Note that $\mathcal{M}_{d, g, n}^{X_{T}}$ is a categorical quotient by a reductive group and the cones constructed in the proof of Proposition 3.9 are all canonical under étale base change. This completes the proof of Theorem 4.2.

Proof of Theorem 4.2'. We still assume $T$ is affine. Let $R$ be a connected component of $R_{2} \pi_{*} \mathbf{Z}_{X_{T}} . R$ is a smooth analytic curve étale over $T$. Let $X_{R}=X_{T} \times_{T} R$ and let $\alpha: R \rightarrow R_{2} \pi_{*} \mathbf{Z}_{X_{R}}$ be the section induced by the component $R$. Note that $\alpha(s) \in H_{2}\left(X_{s}, \mathbf{Z}\right)$ for $s \in R$. Note also that under $H_{2}\left(X_{s}, \mathbf{Z}\right) \rightarrow H_{2}\left(X_{R}, \mathbf{Z}\right)$ all $\alpha(s)$ have identical images. We let $\alpha_{0} \in H_{2}\left(X_{R}, \mathbf{Z}\right)$ be their common images. Let $H_{R}$ be the pull back of the relatively ample line bundle on $X_{T} / T$. Then $\alpha(s) \cdot c_{1}\left(H_{R}\right) \in \mathbf{Z}$ is independent of $s$. We denote it by $d$. Clearly, for any $t \in T$ the disjoint union of $\mathcal{M}_{\alpha(s), g, n}^{X_{s}}$ for all $s \in R$ over $t$ is an open and closed subscheme of $\mathcal{M}_{d, g, n}^{X_{t}}$. Since $\mathcal{M}_{d, g, n}^{X_{t}}$ is projective, it is possible that either $R \rightarrow S$ is finite or the set of $s \in R$ of which $\mathcal{M}_{\alpha(s), g, n}^{X_{s}} \neq \emptyset$ is discrete. We first look into the second situation. From the construction of virtual cycle, it is clear that if $V \subset \mathcal{M}_{d, g, n}^{X_{t}}$ is a connected component, then using the induced tangent-obstruction complex of $V$ we can construct the virtual cycle $[V]^{\text {vir }}$. It follows from the proof of Theorem 4.2 that for any connected component $V$ of $\mathcal{M}_{d, g, n}^{X_{T} / T}$ and immersion $\eta_{t}:\{t\} \rightarrow T$ we have the identity $\left[V_{t}\right]^{\text {vir }}=\eta_{t}^{!}[V]^{\text {vir }}$, where $V_{t}=V \times_{T}\{t\}$. Since $\mathcal{M}_{d, g, n}^{X_{T} / T}$ consists of fibers over a discrete point set of $T, \eta_{t}^{!}[V]^{\text {vir }} \sim_{\text {rat }} 0$ for all $t \in T$. Hence $\left[\mathcal{M}_{\alpha(s), g, n}^{X_{s}}\right]^{\text {vir }}=0$ for all $s \in R$. As to the first situation, since $R \rightarrow S$ is finite, $R$ is algebraic. Hence Theorem 4.2 implies that for any $s \in R,\left[\mathcal{M}_{\alpha(s), g, n}^{X_{s}}\right]^{\text {vir }}=\eta_{s}^{!}\left[\mathcal{M}_{\alpha_{0}, g, n}^{X_{R} / R}\right]^{\text {vir }}$. Theorem $4.2^{\prime}$ then follows from the principle of conservation of number. This proves Theorem $4.2^{\prime}$.

The Gromov-Witten invariants are expected to satisfy a set of relations, as explained in $[\mathrm{KM}]$, [RT1], [RT2]. We state these relations in terms of the virtual moduli cycles. We will provide their proofs except for the composition law, which will be proved in the next section.

We first recall the contraction transformation. For $n \geq 1$ we let $\mathfrak{F}_{\alpha, g, n}^{X} \rightarrow \mathfrak{F}_{\alpha, g, n-1}^{X}$ be the transformation that sends any family $f: \mathcal{X} \rightarrow X$ over $S$ in $\mathfrak{F}_{\alpha, g, n}^{X}(S)$ to the family $f^{\prime}: \mathcal{X}^{\prime} \rightarrow X$, where $\mathcal{X}^{\prime}$ is the curve over $S$ obtained by forgetting the $n$ th labeled section of $\mathcal{X}$ and then stable contracting the resulting $(n-1)$-pointed curve relative to $f$, and $f^{\prime}$ is the unique morphism so that $\mathcal{X} \rightarrow \mathcal{X}^{\prime} \stackrel{\longrightarrow}{\longrightarrow}$ is $\mathcal{X} \stackrel{f}{\longrightarrow} X$. We let $\pi_{n}: \mathcal{M}_{\alpha, g, n}^{X} \rightarrow \mathcal{M}_{\alpha, g, n-1}^{X}$ be the induced morphism. Similarly, we let $p_{n}: \mathcal{M}_{g, n} \rightarrow \mathcal{M}_{g, n-1}$ be the morphism induced by forgetting the last sections.

Theorem 4.3. The virtual moduli cycle $\left[\mathcal{M}_{\alpha, g, n}^{X}\right]^{\text {vir }}$ satisfies the following properties:

(1) $\left[\mathcal{M}_{\alpha, g, n}^{X}\right]^{\text {vir }} \in A_{k} \mathcal{M}_{\alpha, g, n}^{X}$ where $k=(3-\operatorname{dim} X)(g-1)+n+\alpha \cdot c_{1}(X)$.

(2) Let $\sigma \in S_{n}$ be any permutation of $n$ elements and let $\phi_{\sigma}: \mathcal{M}_{\alpha, g, n}^{X} \rightarrow \mathcal{M}_{\alpha, g, n}^{X}$ be the morphism induced by permuting the $n$ marked points of the domains of $f \in \mathcal{M}_{\alpha, g, n}^{X}$. Then $\phi_{\sigma}$ is an isomorphism and $\phi_{\sigma *}\left[\mathcal{M}_{\alpha, g, n}^{X}\right]^{\mathrm{vir}}=\left[\mathcal{M}_{\alpha, g, n}^{X}\right]^{\mathrm{vir}}$. 
(3) The morphism $\pi_{n}: \mathcal{M}_{\alpha, g, n}^{X} \rightarrow \mathcal{M}_{\alpha, g, n-1}^{X}$ is a flat morphism of relative dimension 1. Further

$$
\pi_{n}^{*}\left[\mathcal{M}_{\alpha, g, n-1}^{X}\right]^{\mathrm{vir}}=\left[\mathcal{M}_{\alpha, g, n}^{X}\right]^{\mathrm{vir}} .
$$

(4) Let $\beta \in A^{1} X$ and $e_{n}: \mathcal{M}_{\alpha, g, n}^{X} \rightarrow X$ be the $n$-th evaluation morphism. Then

$$
\pi_{n *}\left(e_{n}^{*} \beta \cdot\left[\mathcal{M}_{\alpha, g, n}^{X}\right]^{\mathrm{vir}}\right)=\operatorname{deg}(\alpha \cdot \beta) \cdot\left[\mathcal{M}_{\alpha, g, n-1}^{X}\right]^{\mathrm{vir}} .
$$

(5) Composition law (see the statement and the proof in the next section).

We remark that what is known as the fundamental class axiom is a direct consequence of (3) of the theorem. Let $\xi_{n}: \mathcal{M}_{\alpha, g, n}^{X} \rightarrow X^{n} \times \mathcal{M}_{g, n}$ be the product of ev $: \mathcal{M}_{\alpha, g, n}^{X} \rightarrow X^{n}$ and the projection $\pi_{n}^{\alpha}: \mathcal{M}_{\alpha, g, n}^{X} \rightarrow \mathcal{M}_{g, n}$. Then another way to describe the GW-invariants is by the homomorphism

$$
I_{\alpha, g, n}^{X}: A^{*}(X)^{\times n} \longrightarrow A_{*} \mathcal{M}_{g, n}
$$

defined by $I_{\alpha, g, n}^{X}(\beta)=\pi_{2 *}\left(\pi_{1}^{*}(\beta)\left(\xi_{*}\left[\mathcal{M}_{\alpha, g, n}^{X}\right]^{\text {vir }}\right)\right)$, where $\pi_{1}$ and $\pi_{2}$ are the first and the second projections of $X^{n} \times \mathcal{M}_{g, n}$. The fundamental class axiom claims that for $n \geq 1$ and $2 g+n \geq 4$, and for any $\beta \in A^{*}(X)^{\times n-1}$, we have

$$
I_{\alpha, g, n}^{X}\left(\beta \times 1_{X}\right)=p_{n}^{*} I_{\alpha, g, n-1}^{X}(\beta),
$$

where $1_{X} \in A^{0}(X)$ is the identity element. We now show that (3) implies (4.3). Consider the commutative diagram

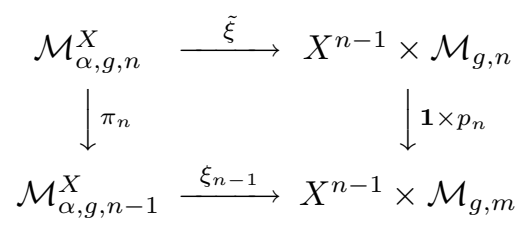

where $1: X^{n-1} \rightarrow X^{n-1}$ is the identity map and $\tilde{\xi}$ is the product of the first $n-1$ evaluation morphisms and the projection $\mathcal{M}_{\alpha, g, n}^{X} \rightarrow \mathcal{M}_{g, n}$. Using the projection formula and the property of cohomology classes [Fu, Definition 17.1], it is direct to check that (4.3) follows from the identity

$$
\left(\mathbf{1} \times p_{n}\right)^{*} \xi_{n-1 *}\left(\left[\mathcal{M}_{\alpha, g, n-1}^{X}\right]^{\mathrm{vir}}\right)=\tilde{\xi}_{*}\left(\left[\mathcal{M}_{\alpha, g, n}^{X}\right]^{\mathrm{vir}}\right) .
$$

In light of (3) of the theorem, to prove (4.4) it suffices to show that for any irreducible variety $Y \subset \mathcal{M}_{\alpha, g, n-1}^{X}$,

$$
\tilde{\xi}_{*} \pi_{n}^{*}([Y])=\left(\mathbf{1} \times p_{n}\right)^{*} \xi_{n-1 *}([Y]) \in Z_{*}\left(X^{n-1} \times \mathcal{M}_{g, n}\right) .
$$

Note that the above square is not necessarily a fiber square. We now prove (4.5). Clearly, $\tilde{\xi}\left(\pi_{n}^{-1}(Y)\right)=\left(\mathbf{1} \times p_{n}\right)^{-1}\left(\xi_{n-1}(Y)\right)$ as sets. Hence it suffices to show that for any irreducible component $W \subset \pi_{n}^{-1}(Y)$ such that $\operatorname{dim} W=\operatorname{dim} \tilde{\xi}(W)$, the coefficient of $[\tilde{\xi}(W)]$ in $\tilde{\xi}_{*} \pi_{n}^{*}([Y])$ is identical to its coefficient in $\left(\mathbf{1} \times p_{n}\right)^{*} \xi_{n-1 *}([Y])$. Let $W \subset \pi_{n}^{-1}(Y)$ be any irreducible component such that $\operatorname{dim} W=\operatorname{dim} \tilde{\xi}(W)$. Let $w \in W$ be a general point associated to the stable map $f_{0}: C_{0} \rightarrow X$ with the marked points $x_{1}, \cdots, x_{n} \in C_{0}$. Let $E_{0}$ be the irreducible component of $C_{0}$ that contains $x_{n}$. Let $\left(\tilde{C}_{0}, \tilde{x}_{1}, \cdots, \tilde{x}_{n-1}\right)$ be the stable contraction of $\left(C_{0}, x_{1}, \cdots, x_{n-1}\right)$. Let $\tilde{E}_{0} \subset \tilde{C}_{0}$ be the image of $E_{0}$. Since $\operatorname{dim} W=\operatorname{dim} \tilde{\xi}(W)$, the map $E_{0} \rightarrow \tilde{E}_{0}$ is generically one-to-one. Let $\tilde{x}_{n} \in \tilde{E}_{0}$ be the image of $x_{n}$. 
Now let $w^{\prime}=\pi_{n}(w), z=\pi_{n}^{\alpha}(w)$ and $z^{\prime}=p_{n}(z)\left(=\pi_{n-1}^{\alpha}\left(w^{\prime}\right)\right)$, and let $G$ be the automorphism group of $\left(\tilde{C}_{0}, \tilde{x}_{1}, \cdots, \tilde{x}_{n-1}\right)$. We claim that there are $G$-schemes $U$, $U^{\prime}, V$ and $V^{\prime}$ and a $G$-equivariant fiber square

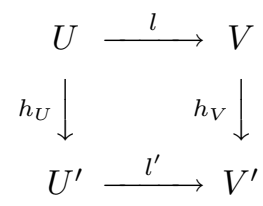

such that their quotients by $G$ are étale neighborhoods of $w \in \mathcal{M}_{\alpha, g, n}^{X}, w^{\prime} \in$ $\mathcal{M}_{\alpha, g, n-1}^{X}, z \in \mathcal{M}_{g, n}$ and $z^{\prime} \in \mathcal{M}_{g, n-1}$, respectively, and that the induced morphisms shown in the following makes the square (4.6) compatible to the middle square in

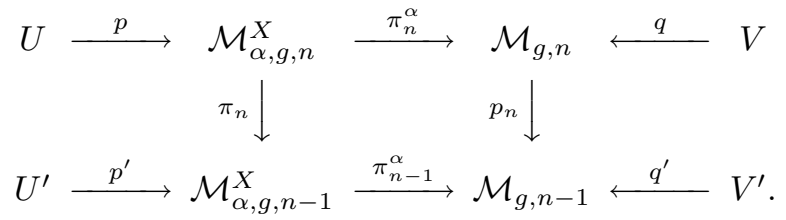

Indeed, we can find a desired $G$-scheme $V^{\prime}$ such that there is a tautological family $\left\{D^{\prime} \subset \mathcal{C}^{\prime}\right\} \in \mathfrak{F}_{g, n-1}\left(V^{\prime}\right)$, where $\mathfrak{F}_{g, n-1}$ is the moduli functor of stable $(n-1)$ pointed curves of genus $g$. Then $V$ can be chosen as an open subset of the total space of $\mathcal{C}^{\prime}$ that contains $\tilde{x}_{n} \in \tilde{C}_{0}$. By shrinking $V^{\prime}$ and $V$ if necessary, we can assume that $V \rightarrow V^{\prime}$ is smooth with connected fibers. As to $U^{\prime}$, we can choose it so that in addition to $U^{\prime} / G$ being an étale neighborhood of $w^{\prime}$ there is a tautological family $\{f: D \subset \mathcal{C} \rightarrow X\} \in \mathfrak{F}_{\alpha, g, n-1}^{X}\left(U^{\prime}\right)$ of which the following holds. First, there is an isomorphism, denoted by $\varphi$, between the stable contraction of $\{D \subset \mathcal{C}\}$ with the pull back of $\left\{D^{\prime} \subset \mathcal{C}^{\prime}\right\}$ under $U^{\prime} \rightarrow V^{\prime}$. Second, for any point $s \in V^{\prime}$ we let $\left\{D_{s}^{\prime} \subset \mathcal{C}_{s}^{\prime}\right\}$ be the fiber of $\left\{D^{\prime} \subset \mathcal{C}^{\prime}\right\}$ over $s$ and let $A_{s}$ be the set of isomorphism classes of pairs $(a, b)$, where $a=\left\{\psi: D_{0} \subset C_{0} \rightarrow X\right\} \in \mathfrak{F}_{\alpha, g, n-1}^{X}(\operatorname{Spec} k)$ such that the stable contraction of $\left\{D_{0} \subset C_{0}\right\}$ is isomorphic to $\left\{D_{s}^{\prime} \subset \mathcal{C}_{s}^{\prime}\right\}$ and $b$ is an isomorphism between the contraction of $\left\{D_{0} \subset C_{0}\right\}$ and the curve $\left\{D_{s}^{\prime} \subset \mathcal{C}_{s}^{\prime}\right\}$. Then the canonical map $l^{\prime-1}(s) \rightarrow A_{s}$ induced by the isomorphism $\varphi$ mentioned in the previous condition is an isomorphism. Now let $U=U^{\prime} \times_{V^{\prime}} V$ and let $\{\tilde{f}: \tilde{D} \subset \tilde{\mathcal{C}} \rightarrow X\}$ be the pull back of $\{f: D \subset \mathcal{C} \rightarrow X\}$ under $U \rightarrow U^{\prime}$. Because $E_{0} \rightarrow \tilde{E}_{0}$ is generically one-to-one, by shrinking $V^{\prime}, V$ and $U^{\prime}$ if necessary, there is a unique section $\tilde{D}_{n}: U \rightarrow \tilde{\mathcal{C}}$ such that $\left\{\tilde{f}: \tilde{D} \cup \tilde{D}_{n} \rightarrow X\right\} \in \mathfrak{F}_{\alpha, g, n}^{X}(U)$ and the stable contraction of $\left\{\tilde{D} \cup \tilde{D}_{n} \subset \tilde{\mathcal{C}}\right\}$ is isomorphic to the pull back of the tautological family over $V^{\prime}$. It is direct to check that the induced map $U / G \rightarrow \mathcal{M}_{\alpha, g, n}^{X}$ makes it an étale neighborhood of $w$. Hence the choice of $U^{\prime}$ 's and $V$ 's satisfy the desired property. With this choice of $U^{\prime}$ and $V$, we can take $U=U^{\prime} \times{ }_{V^{\prime}} V$ which satisfies the desired property. Now let $\tilde{W}$ be any irreducible component of $(\mathbf{1} \times q)^{-1}(\tilde{\xi}(W))$. Then with our choice of $U$, etc., it is clear that if we let $\mu_{[\tilde{W}]}\left((\mathbf{1} \times q)^{*} \tilde{\xi}_{*} \pi_{n}^{*}([Y])\right)$ be the coefficient of $[\tilde{W}]$ in $(\mathbf{1} \times q)^{*} \tilde{\xi}_{*} \pi_{n}^{*}([Y])$, then it is equal to

$$
\begin{aligned}
\mu_{[\tilde{W}]}\left((\widetilde{e v} \times l)_{*} h_{U}^{*} p^{* *}([Y])\right) & =\mu_{[\tilde{W}]}\left(\left(\mathbf{1} \times h_{V}\right)^{*}\left(e v^{\prime} \times l^{\prime}\right) * p^{* *}([Y])\right) \\
& =\mu_{[\tilde{W}]}\left((\mathbf{1} \times q)^{*}\left(\mathbf{1} \times p_{n}\right)^{*} \tilde{\xi}_{*}([Y])\right),
\end{aligned}
$$


where $\widetilde{e v}: U \rightarrow X^{n-1}$ is the composite of $U \rightarrow \mathcal{M}_{\alpha, g, n}^{X}$ with the product of the first $n-1$ evaluation morphisms, and $e v^{\prime}: U^{\prime} \rightarrow X^{n-1}$ is defined similarly. This proves the identity (4.5), and hence (4.6)

Proof of Theorem 4.3. By the construction, the cycle $\left[\mathcal{M}_{\alpha, g, n}^{X}\right]^{\text {vir }}$ is an equidimensional cycle whose dimension is the virtual dimension of $\mathcal{M}_{\alpha, g, n}^{X}$. Using the Riemann-Roch theorem, one calculates that it is exactly the $k$ given in the statement. This proves (1). Also, it is clear that for any $\sigma \in S_{n}$, we have that $\phi_{\sigma}: \mathcal{M}_{\alpha, g, n}^{X} \rightarrow \mathcal{M}_{\alpha, g, n}^{X}$ is an isomorphism of schemes. Since the tangent-obstruction complex of $\mathfrak{F}_{\alpha, g, n}^{X}$ does not depend on the ordering of the marked sections, the virtual moduli cycle will be invariant under $\phi_{\sigma *}$. This proves (2). Next, we prove statement (4) assuming property (3). Since $X$ is smooth, $\beta \in A^{1}$ is the Chern class of a line bundle. By applying the projection formula to the flat morphism $\pi_{n}$, we obtain

$$
\begin{aligned}
\pi_{n *}\left(e_{n}^{*} \beta \cdot\left[\mathcal{M}_{\alpha, g, n}^{X}\right]^{\mathrm{vir}}\right) & =\pi_{n *}\left(e_{n}^{*} \beta \cdot \pi_{n}^{*}\left[\mathcal{M}_{\alpha, g, n-1}^{X}\right]^{\mathrm{vir}}\right) \\
& =\pi_{n *}\left(e_{n}^{*} \beta\right) \cdot\left[\mathcal{M}_{\alpha, g, n-1}^{X}\right]^{\mathrm{vir}} .
\end{aligned}
$$

Because $\pi_{n}$ is flat of relative dimension $1, \pi_{n *}\left(e_{n}^{*} \beta\right)=\operatorname{deg}(\alpha \cdot \beta) \cdot \mathbf{1}$, where $\mathbf{1} \in$ $A^{0} \mathcal{M}_{\alpha, g, n-1}^{X}$ is the identity element. This proves (4).

Now we prove property (3). Let $G$ be the reductive group and $\mathcal{Q}$ be the $G$-scheme mentioned before so that $\mathcal{M}_{\alpha, g, n-1}^{X}$ is the categorical quotient of $\mathcal{Q}$. Let $\mathcal{C}$ be the universal curve over $\mathcal{Q}$. Then $\mathcal{C} \rightarrow \mathcal{Q}$ is flat of relative dimension 1 . It follows from the universal property of $\mathcal{Q}$ that $G$ acts canonically on $\mathcal{C}$ and $\mathcal{M}_{\alpha, g, n}^{X}$ is the categorical quotient of the total scheme of $\mathcal{C}$, denoted $\mathcal{P}$, by $G$. Let $\pi_{n}^{\prime}: \mathcal{M}_{\alpha, g, n}^{X} \rightarrow$ $\mathcal{M}_{\alpha, g, n-1}^{X}$ be the induced morphism. $\pi_{n}^{\prime}$ is the morphism described in the statement (3) of the theorem. Now we argue that $\pi_{n}\left(=\pi_{n}^{\prime}\right)$ is flat of relative dimension 1 . It is obvious that $\pi_{n}$ has relative dimension 1 . Now let $w \in \mathcal{Q}$ be any closed point and let $G_{w} \subset G$ be the stabilizer of $w$. Then there is a $G_{w}$-invariant slice $U \rightarrow \mathcal{Q}$ such that $U / G_{w} \rightarrow \mathcal{M}_{\alpha, g, n-1}^{X}$ is an étale neighborhood. It follows that $\mathcal{C} \times{ }_{\mathcal{Q}} U / G_{w}$ is an étale neighborhood $\mathcal{M}_{\alpha, g, n}^{X}$ and the projection $\mathcal{C} \times{ }_{\mathcal{Q}} U / G_{w} \rightarrow U / G_{w}$ is compatible to the projection $\mathcal{M}_{\alpha, g, n}^{X} \rightarrow \mathcal{M}_{\alpha, g, n-1}^{X}$. Therefore $\pi_{n}$ will be flat if we can show that $\mathcal{C} \times{ }_{\mathcal{Q}} U / G_{w} \rightarrow U / G_{w}$ is flat, which follows from the flatness of $\mathcal{C} \rightarrow \mathcal{Q}$ and that $G_{w}$ is a finite group. This shows that $\pi_{n}$ is flat.

It remains to prove the identity in statement (3). Let $\tilde{f}: \tilde{D} \subset \tilde{\mathcal{C}} \rightarrow X$ be the tautological family over $\mathcal{P}$, where $\tilde{D} \subset \tilde{\mathcal{C}}$ is a family of $n$-pointed curves over $\mathcal{P}$, characterized by the following property. There is a canonical morphism

$$
\pi: \tilde{\mathcal{C}} \longrightarrow \mathcal{C} \times{ }_{\mathcal{Q}} \mathcal{P}
$$

such that the base change $f^{\prime}: \mathcal{C} \times{ }_{\mathcal{Q}} \mathcal{P} \rightarrow X$ of $f$ with the marked divisors $D \times{ }_{\mathcal{Q}} \mathcal{P}$ is the stable contraction of $\tilde{D}_{<n} \subset \tilde{\mathcal{C}}$, where $\tilde{D}_{<n}$ is the first $(n-1)$-marked sections in $\tilde{D}$, relative to $\tilde{f}$; the restriction of $\pi$ to $\tilde{D}_{n}$ is an isomorphism between $\tilde{D}_{n}$ and $\mathcal{C} \times_{\mathcal{Q}} \mathcal{P}$. Let $\xi \in \mathfrak{F}_{\alpha, g, n-1}^{X}(\mathcal{Q})$ (resp. $\tilde{\xi} \in \mathfrak{F}_{\alpha, g, n}^{X}(\mathcal{P})$ ) be the object corresponding to the family $f$ (resp. $\tilde{f}$ ). Following the discussion after Corollary 3.6, to construct $\left[\mathcal{M}_{\alpha, g, n-1}^{X}\right]^{\text {vir }}$, it suffices to find a $G$-linearized locally free sheaf $\mathcal{V}$ of $\mathcal{O}_{\mathcal{Q}}$-modules such that

$$
\mathcal{T}^{2} \mathfrak{F}_{\alpha, g, n-1}^{X}(\xi)\left(\mathcal{O}_{\mathcal{Q}}\right)=\mathcal{E} x t_{\mathcal{C} / \mathcal{Q}}^{2}\left(\left[f^{*} \Omega_{X} \rightarrow \Omega_{\mathcal{C} / \mathcal{Q}}(D)\right], \mathcal{O}_{\mathcal{C}}\right)
$$


is a $G$-linearized quotient of $\mathcal{V}$. As before, we pick a $G$-linearized locally free sheaf $\mathcal{W}_{1}$ of $\mathcal{O}_{\mathcal{C}}$-modules such that $\mathcal{W}_{1}^{-1}$ is sufficiently ample along fibers of $\mathcal{C} \rightarrow \mathcal{Q}$. We then pick a $G$-equivariant quotient homomorphism $\mathcal{W}_{1}^{-1} \rightarrow f^{*} \Omega_{X}$ and let $\mathcal{W}_{2}=$ $\operatorname{ker}\left\{\mathcal{W}_{1} \rightarrow f^{*} \Omega_{X}\right\} . \mathcal{W}_{2}$ is also locally free. It follows that $(4.7)$ is a $G$-equivariant quotient sheaf of

$$
\mathcal{V}:=\mathcal{E} x t_{\mathcal{C} / \mathcal{Q}}^{1}\left(\left[\mathcal{W}_{2} \rightarrow 0\right], \mathcal{O}_{\mathcal{C}}\right)=\mathcal{E} x t_{\mathcal{C} / \mathcal{Q}}^{0}\left(\mathcal{W}_{2}, \mathcal{O}_{\mathcal{C}}\right)
$$

By the discussion after Corollary 3.6, there is a canonical cycle $\left[C^{\mathcal{V}}\right] \in Z_{*} \operatorname{Vect}(\mathcal{V})$ so that the image of its $\mathbf{Q}$-descent over $\mathcal{M}_{\alpha, g, n-1}^{X}$ under the obvious Gysin map is $\left[\mathcal{M}_{\alpha, g, n-1}^{X}\right]^{\text {vir }}$. Now we pick a similar vector bundle over $\mathcal{P}$. We set $\tilde{\mathcal{W}}_{1}=p^{*} \mathcal{W}_{1}$ and $\tilde{\mathcal{W}}_{2}=p^{*} \mathcal{W}_{2}$, where $p$ is the composite map $\operatorname{pr}_{2} \circ \pi: \tilde{\mathcal{C}} \rightarrow \mathcal{C} \times_{\mathcal{Q}} \mathcal{P} \rightarrow \mathcal{C}$. Then $\mathcal{W}_{2} \rightarrow \mathcal{W}_{1} \rightarrow f^{*} \Omega_{X}$ pulls back to $\tilde{\mathcal{W}}_{2} \rightarrow \tilde{\mathcal{W}}_{1} \rightarrow \tilde{f}^{*} \Omega_{X}$. Let

$$
\begin{aligned}
\tilde{\mathcal{V}} & :=\mathcal{E} x t_{\tilde{\mathcal{C}} / \mathcal{P}}^{1}\left(\left[\tilde{\mathcal{W}}_{2} \rightarrow 0\right], \mathcal{O}_{\tilde{\mathcal{C}}}\right) \longrightarrow \mathcal{T}^{2} \mathfrak{F}_{\alpha, g, n}^{X}(\tilde{\xi})\left(\mathcal{O}_{\mathcal{P}}\right) \\
& =\mathcal{E} x t_{\tilde{\mathcal{C}} / \mathcal{P}}^{2}\left(\left[\tilde{f}^{*} \Omega_{X} \rightarrow \Omega_{\tilde{\mathcal{C}} / \mathcal{P}}(\tilde{D})\right], \mathcal{O}_{\tilde{\mathcal{C}}}\right)
\end{aligned}
$$

be the similar quotient homomorphism of sheaves. Because $\pi$ contracts at most one rational curve in each fiber of $\tilde{\mathcal{C}}$ over $\mathcal{P}, \tilde{\mathcal{V}}$ is canonically isomorphic to $p_{n}^{*} \mathcal{V}$, where $p_{n}: \mathcal{P} \rightarrow \mathcal{Q}$ is the projection. We claim that there is a canonical homomorphism $\phi$ making the following diagram commutative:

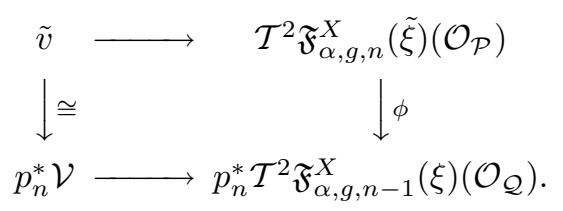

We consider the canonical exact sequences

$$
\begin{array}{ccccc}
\mathcal{E} x t_{\tilde{\mathcal{C}} / \mathcal{P}}^{1}\left(\Omega_{\tilde{\mathcal{C}} / \mathcal{P}}(\tilde{D}), \mathcal{O}_{\tilde{\mathcal{C}}}\right) & \longrightarrow & \mathcal{E} x t_{\tilde{\mathcal{C}} / \mathcal{P}}^{1}\left(\tilde{f}^{*} \Omega_{X}, \mathcal{O}_{\tilde{\mathcal{C}}}\right) & \longrightarrow & \mathcal{E} x t_{\tilde{\mathcal{C}} / \mathcal{P}}^{2}\left(\mathcal{A}^{\bullet}, \mathcal{O}_{\tilde{\mathcal{C}}}\right) \\
& \downarrow^{\phi_{2}} & & & \downarrow^{\phi} \\
\pi_{n}^{*} \mathcal{E} x t_{\mathcal{C} / \mathcal{Q}}^{1}\left(\Omega_{\mathcal{C} / \mathcal{Q}}(D), \mathcal{O}_{\mathcal{C}}\right) & \longrightarrow & \pi_{n}^{*} \mathcal{E} x t_{\mathcal{C} / \mathcal{Q}}^{1}\left(f^{*} \Omega_{X}, \mathcal{O}_{\mathcal{C}}\right) & \longrightarrow & \pi_{n}^{*} \mathcal{E} x t_{\mathcal{C} / \mathcal{Q}}^{2}\left(\mathcal{B}^{\bullet}, \mathcal{O}_{\mathcal{C}}\right)
\end{array}
$$

where $\mathcal{A}^{\bullet}=\left[\tilde{f}^{*} \Omega_{X} \rightarrow \Omega_{\tilde{\mathcal{C}} / \mathcal{P}}(\tilde{D})\right]$ and $\mathcal{B}^{\bullet}=\left[f^{*} \Omega_{X} \rightarrow \Omega_{\mathcal{C} / \mathcal{Q}}(D)\right]$. Clearly, there are canonical homomorphisms $\phi_{1}$ and $\phi_{2}$ as indicated in the above diagram making the left square commutative. Because the two horizontal arrows on the right are surjective, there is a canonical homomorphism $\phi$ making the right square commutative. The commutativity of the diagram (4.8) follows immediately.

Now let $\left[C^{\mathcal{V}}\right] \in Z_{*} \operatorname{Vect}(\mathcal{V})$ (resp. $\left[C^{\tilde{\mathcal{V}}}\right] \in Z_{*} \operatorname{Vect}(\tilde{\mathcal{V}})$ ) be the virtual normal cone cycle constructed in the beginning of section 4 associated to $\mathcal{T}^{\bullet} \mathfrak{F}_{\alpha, g, n-1}^{X}(\xi)$ (resp. $\left.\mathcal{T}^{\bullet} \mathfrak{F}_{\alpha, g, n}^{X}(\tilde{\xi})\right)$, and the quotient homomorphism $\tilde{\mathcal{V}} \rightarrow \mathcal{T}^{2} \mathfrak{F}_{\alpha, g, n}^{X}(\tilde{\xi})\left(\mathcal{O}_{\mathcal{P}}\right)$. Let

$$
\Phi: \operatorname{Vect}(\tilde{\mathcal{V}})=\operatorname{Vect}(\mathcal{V}) \times_{\mathcal{Q}} \mathcal{P} \stackrel{\operatorname{pr}_{1}}{\longrightarrow} \operatorname{Vect}(\mathcal{V})
$$

be the projection. $\Phi$ is flat of relative dimension 1. Now assume that

$$
\Phi^{*}\left[C^{\mathcal{V}}\right]=\left[C^{\tilde{\mathcal{V}}}\right] .
$$

Let $\left[C_{\mathcal{M}}^{\mathcal{V}}\right]$ and $\left[C_{\mathcal{M}}^{\tilde{\mathcal{V}}}\right]$ be the $\mathbf{Q}$-descents of $\left[C^{\mathcal{V}}\right]$ and $\left[C^{\tilde{\mathcal{V}}}\right]$ to $\mathcal{M}_{\alpha, g, n-1}^{X}$ and $\mathcal{M}_{\alpha, g, n}^{X}$ respectively, and let $\operatorname{Vect}_{\mathcal{M}}(\mathcal{V})$ and $\operatorname{Vect}_{\mathcal{M}}(\tilde{\mathcal{V}})$ be the $\mathbf{Q}$-descents of $\operatorname{Vect}(\mathcal{V})$ and 
$\operatorname{Vect}(\tilde{\mathcal{V}})$ respectively. Then

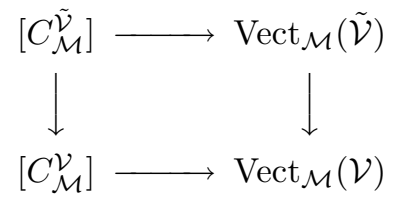

is a pull back diagram. Therefore,

$$
\left[\mathcal{M}_{\alpha, g, n}^{X}\right]^{\text {vir }}=\eta_{\tilde{V}}^{*}\left[C_{\mathcal{M}}^{\tilde{\mathcal{V}}}\right]=\pi_{n}^{*} \eta_{V}^{*}\left[C_{\mathcal{M}}^{\mathcal{V}}\right]=\left[\mathcal{M}_{\alpha, g, n-1}^{X}\right]^{\text {vir }},
$$

where $\eta_{\tilde{V}}$ and $\eta_{V}$ are the 0 -sections of $\operatorname{Vect}(\tilde{\mathcal{V}})$ and $\operatorname{Vect}(\mathcal{V})$.

We now prove the identity (4.9). We first note that there is a largest subscheme $\Sigma \subset \mathcal{P}$ characterized by the property that the restriction of the contraction morphism

$$
\left.\pi\right|_{\tilde{\mathcal{C}} \times_{\mathcal{P}} \Sigma}: \tilde{\mathcal{C}} \times_{\mathcal{P}} \Sigma \longrightarrow \mathcal{C} \times_{\mathcal{Q}} \Sigma
$$

contracts a $\mathbf{P}^{1}$-bundle over $\Sigma$. Since the image of any of these $\mathbf{P}^{1}$ in $\mathcal{C}$ must be either one of the marked points or one of the singular points of the curves in this family, the restriction of $p_{n}: \mathcal{P} \rightarrow \mathcal{Q}$ to $\Sigma$ is a finite morphism. Let $w \in \mathcal{P}$ be any closed point over $z \in \mathcal{Q}$. Let $\tilde{C}$ and $C$ be the restriction of $\tilde{\mathcal{C}}$ and $\mathcal{C}$ to $w$ and $z$ respectively. We let $\tilde{\varphi}: \tilde{C} \rightarrow X$ and $\varphi: C \rightarrow X$ be the corresponding morphisms, and $\tilde{D} \subset \tilde{C}$ and $D \subset C$ be the corresponding marked points, respectively. Then $(f, C, D)$ is the stable contraction of $(\tilde{f}, \tilde{C}, \tilde{D}<n)$ relative to $\tilde{f}$. We first consider the situation where $\tilde{C}$ is isomorphic to $C$. Namely, $\tilde{f} \in \mathcal{P}-\Sigma$. Clearly

$$
\phi_{w}:=\phi \otimes k(w): \operatorname{Ext}_{\tilde{C}}^{2}\left(\left[\tilde{\varphi}^{*} \Omega_{X} \rightarrow \Omega_{\tilde{C}}(\tilde{D})\right], \mathcal{O}_{\tilde{C}}\right) \cong \operatorname{Ext}_{C}^{2}\left(\left[\varphi^{*} \Omega_{X} \rightarrow \Omega_{C}(D)\right], \mathcal{O}_{C}\right)
$$

is an isomorphism. We claim that the obstruction theory to deformations of $\tilde{\varphi}$ is identical to the obstruction theory to deformations of $\varphi$. Indeed, let $B$ be any Artin ring with residue field $k$ and let $I \subset B$ be an ideal annihilated by the maximal ideal of $B$. Let $B_{0}=B / I$ and let $\tilde{\varphi}_{0}: \tilde{D}_{0} \subset \tilde{C}_{0} \rightarrow X$ be a flat family over Spec $B_{0}$ whose restriction to the fiber over Spec $k$ is $\tilde{\varphi}$. Let $\tilde{o}$ be the obstruction to extending $\tilde{\varphi}_{0}$ to families over Spec $B$. Similarly, we let $\varphi_{0}: D_{0} \subset C_{0} \rightarrow X$ be the stable contraction of $\tilde{\varphi}_{0}: \tilde{D}_{0,<n} \subset \tilde{C}_{0} \rightarrow X$ and let $o$ be the obstruction to extending $\varphi_{0}$ to families over Spec $B$. By our description of the obstruction theory of $\mathfrak{F}_{\alpha, g, n}^{X}$ in section 1 , $\phi_{w}(\tilde{o})=o$. Now let

$$
T_{i, w}=\operatorname{Ext}_{\tilde{C}}^{i}\left(\left[\tilde{\varphi}^{*} \Omega_{X} \rightarrow \Omega_{\tilde{C}}(\tilde{D})\right], \mathcal{O}_{\tilde{C}}\right) \quad \text { and } \quad T_{i, z}=\operatorname{Ext}_{C}^{i}\left(\left[\varphi^{*} \Omega_{X} \rightarrow \Omega_{C}(D)\right], \mathcal{O}_{C}\right) .
$$

Note that in our situation, $T_{2, z} \cong T_{2, w}$ and $T_{1, z}$ is canonically a quotient vector space of $T_{1, w}$ with $\operatorname{dim} T_{1, w}=\operatorname{dim} T_{1, z}+1$. Let $h: T_{1, w} \rightarrow T_{1, z}$ be the projection. Let $f_{z} \in \hat{\operatorname{Sym}} \bullet\left(T_{1, z}^{\vee}\right) \otimes_{k} T_{2, z}$ be a Kuranishi map of $w$. Then $f_{w}:=h^{*}\left(f_{z}\right) \in$ $\hat{\operatorname{Sym}} \bullet\left(T_{1, w}^{\vee}\right) \otimes_{k} T_{2, w}$ is a Kuranishi map of $w$. Now let

$$
\hat{z}:=\operatorname{Spec} \hat{\operatorname{Sym}} \bullet\left(T_{1, z}^{\vee}\right) /\left(f_{z}\right) \subset \hat{T}:=\operatorname{Spec} \hat{\operatorname{Sym}} \bullet\left(T_{1, z}^{\vee}\right)
$$

and let

$$
\hat{w}:=\operatorname{Spec} \hat{\operatorname{Sym}} \bullet\left(T_{1, w}^{\vee}\right) /\left(f_{w}\right) \subset \hat{S}:=\operatorname{Spec} \hat{\operatorname{Sym}} \bullet\left(T_{1, w}^{\vee}\right) .
$$

Because the normal cones are canonical under flat base change [Vi], the normal cone

$$
\left[C^{f_{w}}\right]:=\left[C_{\hat{w} / \hat{S}}\right] \in Z_{*}\left(\operatorname{Vect}\left(T_{2, w}\right) \times_{k} \hat{w}\right)
$$


is the pull back of

$$
\left[C^{f_{z}}\right]:=\left[C_{\hat{z} / \hat{T}}\right] \in Z_{*}\left(\operatorname{Vect}\left(T_{2, z}\right) \times_{k} \hat{z}\right)
$$

under the canonical flat morphism $\hat{w} \rightarrow \hat{z}$. Hence, if we let $U=\mathcal{P}-\Sigma$, then

$$
R_{U}^{*}\left(\Phi^{*}\left(\left[C^{\mathcal{V}}\right]\right)\right)=R_{U}^{*}\left(\left[C^{\tilde{\mathcal{V}}}\right]\right) \in Z_{*}\left(\operatorname{Vect}(\tilde{\mathcal{V}}) \times_{\mathcal{P}} U\right),
$$

where $R_{U}: \operatorname{Vect}(\tilde{\mathcal{V}}) \times_{\mathcal{P}} U \rightarrow \operatorname{Vect}(\tilde{\mathcal{V}})$ is the open immersion. Since both $\Phi^{*}\left(\left[C^{\mathcal{V}}\right]\right)$ and $\left[C^{\tilde{\mathcal{V}}}\right]$ are cycles of identical dimensions, to show $\Phi^{*}\left[C^{\mathcal{V}}\right]=\left[C^{\tilde{\mathcal{V}}}\right]$, it suffices to show that no irreducible components of $\left[C^{\tilde{\mathcal{V}}}\right]$ are contained in $\operatorname{Vect}(\tilde{\mathcal{V}}) \times_{\mathcal{P}} \Sigma$.

Now we consider $w \in \Sigma$. Let $T_{1, w}$ and $T_{2, w}$ be as before, and let

$$
f_{w} \in \hat{\operatorname{Sym}} \bullet\left(T_{1, w}^{\vee}\right) \otimes_{k} T_{2, w}
$$

be a Kuranishi map of $w$. By the definition of Kuranishi families, there is an associated family

$$
F: \mathcal{X} \longrightarrow X \quad \text { and } \quad \mathcal{D} \subset \mathcal{X}
$$

of stable morphisms over $\hat{w}$. We let $\mathcal{X}^{\prime}$ over $\hat{w}$ be the resulting curve obtained by stable contracting $\mathcal{D}_{<n} \subset \mathcal{X}$ relative to $F$. Let $\mathcal{X} \rightarrow \mathcal{X}^{\prime}$ be the contraction morphism and let $\hat{\Sigma} \subset \hat{w}$ be $g^{-1}(\Sigma)$, where $g: \hat{w} \rightarrow \mathcal{P}$ is a morphism such that $F$ is the pull back of the tautological family $\tilde{f}$ over $\mathcal{P}$. It follows that $\left[C^{\tilde{\mathcal{V}}}\right]$ has no components supported on $\Sigma$ if and only if for any $w \in \Sigma$ the cycle $\left[C_{\hat{w} / \hat{S}}\right]$ has no components supported over $\hat{\Sigma}$.

Now we prove this statement. Let $z=\pi_{n}(w) \in \mathcal{Q}$, let $T_{1, z}$ and $T_{2, z}$ be as before and let $f_{z} \in \hat{\operatorname{Sym}} \bullet\left(T_{1, z}^{\vee}\right) \otimes_{k} T_{2, z}$ be a Kuranishi map of $z$. Similarly, we let

$$
G: \mathcal{Y} \longrightarrow X \quad \text { and } \quad \mathcal{E} \subset \mathcal{Y}
$$

be the associated family of stable morphisms over $\hat{z}$. It follows that there is a canonical isomorphism $\mathcal{X}^{\prime} \times \hat{w}\{w\} \cong \mathcal{Y} \times \hat{z}\{z\}$. Now let $r \in \mathcal{Y} \times_{\hat{z}}\{z\}$ be the image of the $\mathbf{P}^{1} \subset \mathcal{X} \times_{\hat{w}}\{w\}$ that was contracted under $\mathcal{X} \rightarrow \mathcal{X}^{\prime}$. Let $\hat{r}$ be the formal completion of the total scheme of $\mathcal{Y}$ along $r$. It follows that $\hat{r}$ is isomorphic to $\hat{w}$. Without loss of generality, we can assume that $\mathcal{Y} / \hat{z}$ can be extended to a family of nodal curves, say $\tilde{\mathcal{Y}}$, over $\hat{T}$. In case the total space of $\tilde{\mathcal{Y}}$ is smooth at $r$, we let $\hat{T}^{\prime}=\hat{T}$ and let $\hat{R}$ be the formal completion of $\tilde{\mathcal{Y}}$ along $r$. Otherwise, because $\tilde{\mathcal{Y}} \rightarrow \hat{T}$ is a flat family of nodal curves, by embedding $\hat{T}$ in $\hat{T}^{\prime}=\operatorname{Spec} \operatorname{Sym} \bullet\left(T_{1, z} \oplus k\right)$, we can assume that $\tilde{\mathcal{Y}} / \hat{T}$ extends to an $\tilde{\mathcal{Y}}^{\prime}$ over $\hat{T}^{\prime}$ so that the total space of $\tilde{\mathcal{Y}}^{\prime}$ is smooth at $r$. Then we let $\hat{R}$ be the formal completion of $\tilde{\mathcal{Y}}^{\prime}$ along $r$. It follows that $\hat{w} \cong \hat{r}$ and $\operatorname{dim} \hat{R}=\operatorname{dim} T_{1, w}$. Let $p: \hat{R} \rightarrow \hat{T}^{\prime}$ be the induced projection. Because $p$ is flat and $\hat{r}=\hat{z} \times \hat{T}^{\prime} \hat{R}$,

$$
C_{\hat{z} / \hat{T}^{\prime}} \times{ }_{\hat{z}} \hat{r} \cong C_{\hat{r} / \hat{R}}
$$

However, because $\hat{r} \cong \hat{w}$ and $\operatorname{dim} \hat{R}=\operatorname{dim} \hat{S}, \hat{r} \cong \hat{w}$ extends to an isomorphism $\hat{R} \cong \hat{S}$. Therefore, there is an isomorphism $C_{\hat{r} / \hat{R}} \cong C_{\hat{w} / \hat{S}}$. Finally, because the restriction of the composite $\hat{w} \cong \hat{r} \stackrel{p}{\rightarrow} \hat{z}$ to $\hat{\Sigma} \subset \hat{w}$ is finite, where $\hat{\Sigma}$ is the formal completion of $\Sigma$ along $w, C_{\hat{r} / \hat{R}}$, and hence $C_{\hat{w} / \hat{S}}$, has no components supported over $\hat{\Sigma}$. This proves that $\left[C^{\tilde{\mathcal{V}}}\right]$ has no components supported over $\Sigma$ and hence $\left[C^{\tilde{\mathcal{V}}}\right]$ is isomorphic to the pull back of $\left[C^{\mathcal{V}}\right]$ as subcone cycles in $\operatorname{Vect}(\tilde{\mathcal{V}})$. This completes the proof of the theorem. 


\section{Composition laws of GW-invariants}

The goal of this section is to prove the composition laws of the GW invariants.

Before we state and prove the theorem, let us introduce some conventions which we will use. In $[\mathrm{Kn}]$, Knudsen described various clutching morphisms, of which two are basic to the composition laws. We fix a partition $g_{1}+g_{2}=g$ and a partition $n_{1}+n_{2}=n$ once and for all. We always assume that $g_{1}, g_{2}, n_{1}$ and $n_{2}$ are nonnegative. Let $\mathfrak{F}_{g, n}$ be the moduli functor of stable $n$-pointed genus $g$ curves and let $\mathcal{M}_{g, n}$ be its coarse moduli scheme. Let $S$ be any scheme, let $\xi_{1} \in \mathfrak{F}_{g_{1} n_{1}+1}(S)$ be represented by the family $C_{1}$ and let $\xi_{2} \in \mathfrak{F}_{g_{2}, n_{2}+1}(S)$ be represented by $C_{2}$. We let $\xi \in \mathfrak{F}_{g, n}$ be the object represented by the family $C$ obtained by identifying the last marked section of $C_{1}$ with the first marked section of $C_{2}$, and set its $n$-marked sections to be the first $n_{1}$ sections of $C_{1}$ followed by the last $n_{2}$ sections of $C_{2}$. In this way, we obtain the so-called clutching transformation

$$
\tilde{\tau}_{g_{\bullet} n \bullet}: \mathfrak{F}_{g_{1}, n_{1}+1}(S) \times \mathfrak{F}_{g_{2}, n_{1}+1}(S) \longrightarrow \mathfrak{F}_{g, n}(S) .
$$

If there is no confusion, we will write $C=\tilde{\tau}_{g_{\bullet} n}\left(C_{1}, C_{2}\right)$. We denote the induced morphism on their moduli schemes by

$$
\tau_{g \bullet n \bullet}: \mathcal{M}_{g_{1}, n_{1}+1} \times \mathcal{M}_{g_{2}, n_{2}+1} \longrightarrow \mathcal{M}_{g, n}
$$

and call it the clutching morphism. The other clutching morphism is defined as follows. Given $\xi \in \mathfrak{F}_{g-1, n+2}(S)$ represented by the curve $C$, we obtain a new curve by identifying the last two marked sections of $C$ and keep the initial $n$ sections. The resulting curve is in $\mathfrak{F}_{g, n}(S)$. We denote this transformation by $\tilde{\tau}_{g-1, n+2}$ and denote the morphism between their moduli schemes by $\tau_{g-1, n+2}: \mathcal{M}_{g-1, n+2} \longrightarrow \mathcal{M}_{g, n}$.

Theorem 5.1. Let $X$ be any smooth projective variety. Assume that $\tau_{i}, \tilde{\tau}_{i} \in$ $H^{*}(X)$ are elements so that $[\Delta]^{\vee}=\sum_{i=1}^{k} \tau_{i} \otimes \tilde{\tau}_{i}$ is the Künneth decomposition of the Poincaré dual of the class $[\Delta]$, where $\Delta \subset X \times X$ is the diagonal. Then

(1) For any $h_{1} \in H_{*}\left(\mathcal{M}_{g_{1}, n_{1}+1}\right)$ and $h_{2} \in H_{*}\left(\mathcal{M}_{g_{2}, n_{2}+1}\right)$, we have

$$
\begin{aligned}
& \psi_{\alpha, g, n}^{X}\left(\xi_{1}, \cdots, \xi_{n}, \tau_{g_{\bullet} n_{\bullet} *}\left(h_{1} \times h_{2}\right)^{\vee}\right) \\
= & \sum_{\alpha_{1}+\alpha_{2}=\alpha} \sum_{i=1}^{k} \psi_{\alpha_{1}, g_{1}, n_{1}+1}^{X}\left(\xi_{1}, \cdots, \xi_{n_{1}}, \tau_{i}, h_{1}^{\vee}\right) \cdot \psi_{\alpha_{2}, g_{2}, n_{2}+1}^{X}\left(\xi_{n_{1}+1}, \cdots, \xi_{n}, \tilde{\tau}_{i}, h_{2}^{\vee}\right) .
\end{aligned}
$$

(2) For any $h \in H_{*}\left(\mathcal{M}_{g-1, n+2}\right)$, we have

$$
\psi_{\alpha, g, n}^{X}\left(\xi_{1}, \cdots, \xi_{n}, \tau_{g-1, n+2 *}(h)^{\vee}\right)=\sum_{i=1}^{k} \psi_{\alpha, g-1, n+2}^{X}\left(\xi_{1}, \cdots, \xi_{n}, \tau_{i}, \tilde{\tau}_{i}, h^{\vee}\right) .
$$

We now state the composition law at the level of cycles. The numerical version above is a direct consequence of it. Let $\tilde{\pi}_{n}^{\alpha}: \mathfrak{F}_{\alpha, g, n}^{X} \rightarrow \mathfrak{F}_{g, n}$ be the transformation that sends any map in $\mathfrak{F}_{\alpha, g, n}^{X}(S)$ to the curve obtained by first forgetting the map and then stable contract the remaining $n$-pointed curve. Let $\pi_{n}^{\alpha}: \mathcal{M}_{\alpha, g, n}^{X} \rightarrow \mathcal{M}_{g, n}$ be the morphism between the respective moduli schemes. 
Theorem 5.2. (1) Assume $n_{1}, n_{2}>0$. We form the fiber products

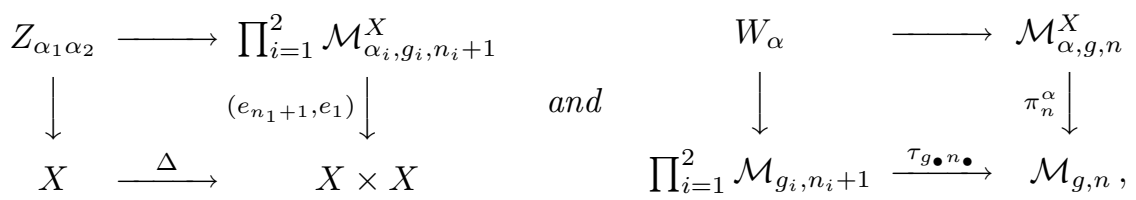

where $X \stackrel{\Delta}{\longrightarrow} X \times X$ is the diagonal and $e_{k}: \mathcal{M}_{\alpha, g, n}^{X} \rightarrow X$ is the $k$-th evaluation morphism. Then there is a canonical morphism $\Psi: \cup_{\alpha_{1}+\alpha_{2}=\alpha} Z_{\alpha_{1} \alpha_{2}} \rightarrow W_{\alpha}$ that is finite, unramified and dominant. Further,

$$
\Psi_{*}\left(\sum_{\alpha_{1}+\alpha_{2}=\alpha} \Delta^{!}\left[\prod_{i=1}^{2} \mathcal{M}_{\alpha_{i}, g_{i}, n_{i}+1}^{X}\right]^{\mathrm{vir}}\right)=\left(\tau_{g_{\bullet}} n_{\bullet}\right)^{!}\left[\mathcal{M}_{\alpha, g, n}^{X}\right]^{\mathrm{vir}} .
$$

(2) Let $Z_{1}$ and $Z_{2}$ be defined by the following fiber diagrams:

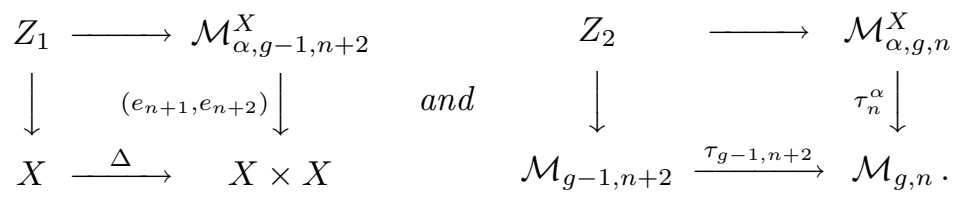

Then the canonical morphism $\Phi: Z_{1} \rightarrow Z_{2}$ is finite, unramified and dominant. Further,

$$
\Phi_{*}\left(\Delta^{!}\left[\mathcal{M}_{\alpha, g-1, n+2}^{X}\right]^{\mathrm{vir}}\right)=\left(\tau_{g-1, n+2}\right)^{!}\left[\mathcal{M}_{\alpha, g, n}^{X}\right]^{\mathrm{vir}} .
$$

We will refer to them as the first composition law and the second composition law.

We will give a detailed proof for the first composition law in this paper. The proof for the second is almost identical The only difference with the proof of the second law is that when $n_{1}, n_{2}>0$ the clutching morphism $\tau_{g_{\bullet}} n_{\bullet}$ is a closed immersion while $\tau_{g-1, n+2}$ are only locally closed embeddings. Some modifications are required for these cases, which we will mention at the end of this section.

We first observe that by property (4) of Theorem 4.3, the first composition law for $\mathcal{M}_{\alpha, g, n}^{X}$ can be obtained from that of $\mathcal{M}_{\alpha, g, n+1}^{X}$. Therefore to prove the first composition law it suffices to prove the case when $n_{1}, n_{2}>0$, which we will assume from now on.

Before we explain the strategy of the proof, let us first recall the notion of $\mathbf{Q}$ schemes which is a straightforward generalization of $\mathbf{Q}$-varieties in $[\mathrm{Mu}]$.

Definition 5.3. We define a $\mathbf{Q}$-scheme to be a scheme $Z$ with the following data.

(1) A finite atlas of charts $Z_{\beta} \stackrel{\pi_{\beta}}{\rightarrow} Z_{\beta} / G_{\beta} \stackrel{p_{\beta}}{\rightarrow} Z$, where $p_{\beta}$ are étale, $G_{\beta}$ is a finite group acting faithfully on a quasi-projective scheme $Z_{\beta}$ and $Z=\cup\left(\operatorname{Im} p_{\beta}\right)$.

(2) For any pair of indices $\alpha$ and $\beta$, there is a chart $Z_{\alpha \beta}$ with the group $G_{\alpha \beta}=$ $G_{\alpha} \times G_{\beta}$ such that there are equivariant finite étale $Z_{\alpha \beta} \rightarrow Z_{\alpha}, Z_{\alpha \beta} \rightarrow Z_{\beta}$ commuting with projection $Z_{\alpha}, Z_{\beta}, Z_{\alpha \beta} \rightarrow X$ such that $\operatorname{Im}\left(p_{\alpha \beta}\right)=\operatorname{Im}\left(p_{\alpha}\right) \cap$ $\operatorname{Im}\left(p_{\beta}\right)$.

(3) For any triple $\alpha, \beta$ and $\gamma$, there is a chart $Z_{\alpha \beta \gamma}$ with the group $G_{\alpha \beta}=G_{\alpha} \times$ $G_{\beta} \times G_{\gamma}$ such that there are equivariant finite étale morphisms from $Z_{\alpha \beta \gamma}$ to $Z_{\alpha}, Z_{\beta}$ and $Z_{\gamma}$ such that in addition to $\operatorname{Im}\left(p_{\alpha \beta \gamma}\right)=\operatorname{Im}\left(p_{\alpha}\right) \cap \operatorname{Im}\left(p_{\beta}\right) \cap \operatorname{Im}\left(p_{\gamma}\right)$, 
the diagram

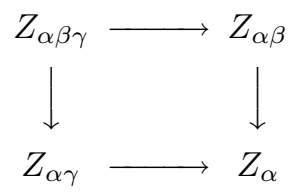

and the other two obtained by permuting the indices are all commutative.

It is known that $\mathcal{M}_{\alpha, g, n}^{X}$ is a projective $\mathbf{Q}$-scheme. Let $\beta \in \mathcal{M}_{\alpha, g, n}^{X}$ be any point associated to the morphism $f: C \rightarrow X$ and let $G_{\beta}$ be its automorphism group. Then we can find an affine open $Z_{\beta}$ acted on by $G_{\beta}$, a $G_{\beta}$-equivariant family $\xi_{\beta} \in$ $\mathfrak{F}_{\alpha, g, n}^{X}\left(Z_{\beta}\right)$ of stable morphisms so that the classifying morphism $Z_{\beta} / G_{\beta} \rightarrow \mathcal{M}_{\alpha, g, n}^{X}$ induced by the family $\xi_{\beta}$ is an étale neighborhood of $\beta \in \mathcal{M}_{\alpha, g, n}^{X}$. For a pair $\beta$ and $\beta^{\prime}$, we can take $Z_{\beta \beta^{\prime}}=\mathbf{I s o}_{Z_{\beta} \times Z_{\beta^{\prime}}}\left(\pi_{1}^{*} \xi_{\beta}, \pi_{2}^{*} \xi_{\beta^{\prime}}\right)$ (see [DM, p. 84]). For a triple $\beta, \beta^{\prime}$ and $\beta^{\prime \prime}$, we can take $Z_{\beta \beta^{\prime} \beta^{\prime \prime}}$ as a subscheme of $Z_{\beta} \times Z_{\beta^{\prime}} \times Z_{\beta^{\prime \prime}}$ defined similarly. One can introduce the notion of $\mathbf{Q}$-sheaves of $\mathcal{O}_{\mathcal{M}_{\alpha, g, n}^{X}}^{X}$-modules, or $\mathbf{Q}$-complex, in the obvious way. A $\mathbf{Q}$-sheaf is a collection of $G_{\beta}$-equivariant sheaves $\mathcal{F}_{\beta}$ on $Z_{\beta}$ with isomorphism $\mathcal{F}_{\beta} \otimes_{\mathcal{O}_{z_{\beta}}} \mathcal{O}_{Z_{\beta \beta^{\prime}}} \cong \mathcal{F}_{\beta^{\prime}} \otimes_{Z_{\beta^{\prime}}} \mathcal{O}_{Z_{\beta \beta^{\prime}}}$, satisfying the cocycle condition over the triple overlaps. Given a $\mathbf{Q}$-locally free sheaf $\mathcal{E}$ on $\mathcal{M}_{\alpha, g, n}^{X}$, we can define the Chern class $c_{i}(\mathcal{E})$ as a cohomological class with rational coefficients, mimicking the similar definition over Deligne-Mumford stacks. Lastly, if $L$ is a $\mathbf{Q}$-line bundle on $\mathcal{M}_{\alpha, g, n}^{X}$ and $s$ is a section of $L$, by which we mean a collection of $s_{\beta} \in H^{0}\left(Z_{\beta}, L_{\beta}\right)$ satisfying the obvious compatibility condition on double overlaps, we can define the localized first Chern class, denoted $c_{1}([L, s])$, using the normal cone construction, mimicking the construction in $[\mathrm{Fu}, \S 14.1]$. One key observation, which can be checked directly, is the following. Assume that for some integer $k, L^{\otimes k}$ is a line bundle on $\mathcal{M}_{\alpha, g, n}^{X}$. Then $c_{1}\left(L^{\otimes k}\right)=k \cdot c_{1}([L, s])$.

The strategy to prove the first composition law is quite simple, at least conceptually. For any partition $\alpha_{1}+\alpha_{2}=\alpha$, we let $\tau_{\alpha_{1} \alpha_{2}}: Z_{\alpha_{1} \alpha_{2}} \rightarrow \mathcal{M}_{\alpha, g, n}^{X}$ be the clutching morphism that sends pairs $f_{1}: C_{1} \rightarrow X$ and $f_{2}: C_{2} \rightarrow X$ to $f: C \rightarrow X$, where $C=\tilde{\tau}_{g_{\bullet} n \bullet}\left(C_{1}, C_{2}\right)$ and $f$ is the obvious induced morphism. Since $n_{1}, n_{2}>0, \tau_{\alpha_{1} \alpha_{2}}$ is a closed immersion. We will first introduce a $\mathbf{Q}$-line bundle $L_{\alpha_{1} \alpha_{2}}$ on $\mathcal{M}_{\alpha, g, n}^{X}$ and a section $f_{\alpha_{1} \alpha_{2}}$ such that the $\mathbf{Q}$-subscheme defined by $f_{\alpha_{1} \alpha_{2}}=0$ is the image scheme of $W_{\alpha_{1} \alpha_{2}} \triangleq \tau_{\alpha_{1} \alpha_{2}}\left(Z_{\alpha_{1} \alpha_{2}}\right)$. We will construct a $\mathbf{Q}$-line bundle $L_{n}$ on $\mathcal{M}_{g, n}$ and a section $f_{n}$ so that $f_{n}^{-1}(0)$ is the image scheme of the clutching morphism $\tau_{g_{\bullet}} n_{\bullet}$. The significance of these line bundles and sections are given in the following lemma.

Lemma 5.4. There is an isomorphism of $\mathbf{Q}$-line bundles

$$
\bigotimes_{\alpha_{1}+\alpha_{2}=\alpha} L_{\alpha_{1} \alpha_{2}} \cong\left(\pi_{n}^{\alpha}\right)^{*} L_{n}
$$

so that under this isomorphism, we have

$$
\prod_{\alpha_{1}+\alpha_{2}=\alpha} f_{\alpha_{1} \alpha_{2}}=\left(\pi_{n}^{\alpha}\right)^{*} f_{n} .
$$

It follows that $\tau_{g_{\bullet} n_{\bullet}}^{!}\left[\mathcal{M}_{\alpha, g, n}^{X}\right]^{\text {vir }}$ is

$$
c_{1}\left(\left(\pi_{n}^{\alpha}\right)^{*}\left[L_{n}, f_{n}\right]\right)\left[\mathcal{M}_{\alpha, g, n}^{X}\right]^{\mathrm{vir}}=\sum_{\alpha_{1}+\alpha_{2}=\alpha} c_{1}\left(\left[L_{\alpha_{1} \alpha_{2}}, f_{\alpha_{1} \alpha_{2}}\right]\right)\left[\mathcal{M}_{\alpha, g, n}^{X}\right]^{\mathrm{vir}} .
$$


In light of Proposition 3.9, the composition law will follow from

$$
c_{1}\left(\left[L_{\alpha_{1} \alpha_{2}}, f_{\alpha_{1} \alpha_{2}}\right]\right)=\left[Z_{\alpha_{1} \alpha_{2}}\right]^{\text {vir }}=\Delta^{!}\left(\left[\mathcal{M}_{\alpha_{1}, g_{1}, n_{1}+1}^{X} \times \mathcal{M}_{\alpha_{2}, g_{2}, n_{2}+1}^{X}\right]^{\text {vir }}\right),
$$

which will follow from establishing the compatibility of the tangent-obstruction complexes of the corresponding functors.

We now give the details of the proof. We first recall some known facts. In this section, we will view $\mathcal{M}_{\alpha, g, n}^{X}$ and $\mathcal{M}_{g, n}$ as Q-moduli schemes. We let

$$
\left\{\left(V_{\beta}, V_{\beta} / G_{\beta}, \mathcal{C}_{V_{\beta}}\right)\right\}_{\beta \in \Lambda_{n}}
$$

be an atlas of $\mathcal{M}_{g, n}$, where $\mathcal{C}_{V_{\beta}}$ are the tautological families over $V_{\beta}$, and let

$$
\left\{\left(W_{\beta}, W_{\beta} / K_{\beta}, F_{W_{\beta}}, \mathcal{C}_{W_{\beta}}\right)\right\}_{\beta \in \Lambda_{\alpha}}
$$

be an atlas of $\mathcal{M}_{\alpha, g, n}^{X}$, where $F_{W_{\beta}}: \mathcal{C}_{W_{\beta}} \rightarrow X$ are the tautological families over $W_{\beta}$. Assume that for some $w \in W_{\beta}$ and $u \in V_{\beta^{\prime}},\left.\mathcal{C}_{V_{\beta^{\prime}}}\right|_{u}$ is isomorphic to the stable contraction (i.e. $\left.\tilde{\pi}_{n}^{\alpha}\right)$ of $\left.\mathcal{C}_{W_{\beta}}\right|_{w}$; then there is an étale neighborhood $\left(\tilde{w}, \tilde{W}_{\beta}\right) \stackrel{\pi}{\rightarrow}\left(w, W_{\beta}\right)$ such that there is a morphism $\tilde{W}_{\beta} \stackrel{\phi}{\longrightarrow} U_{\beta^{\prime}}$ induced by the transformation $\tilde{\pi}_{n}^{\alpha}$, where the family over $\tilde{W}_{\beta}$ is the pull back from $W_{\beta}$. Similarly, let $\beta_{1}$ and $\beta_{2}$ be any two indices and let $\psi_{1}: \tilde{W}_{\beta_{1}} \rightarrow V_{\beta_{1}^{\prime}}$ and $\psi_{2}: \tilde{W}_{\beta_{2}} \rightarrow V_{\beta_{2}^{\prime}}$ be the so-defined morphisms. Then there is a canonical lifting $\psi_{12}: \tilde{W}_{\beta_{1} \beta_{2}} \rightarrow V_{\beta_{1}^{\prime} \beta_{2}^{\prime}}$. This follows immediately from the way the double overlaps are constructed. Another property we need is the following. Let $S$ be any scheme and $\mathcal{X}$ be a family in $\mathfrak{F}_{g, n}(S)$. Assume that for some $s \in S$ and $u \in V_{\beta}$, the restriction $\left.\mathcal{X}\right|_{s}$ is isomorphic to the restriction $\left.\mathcal{C}_{V_{\beta}}\right|_{u}$. Then there is an étale neighborhood $\left(s_{0}, S_{0}\right) \stackrel{\pi}{\rightarrow}(s, S)$ such that there is a morphism $\varphi:\left(s_{0}, S_{0}\right) \rightarrow V_{\beta}$ such that $\varphi^{*} \mathcal{C}_{V_{\beta}} \cong \pi^{*} \mathcal{X}$ and that their restriction to $s_{0}$ is exactly the isomorphism $\left.\left.\mathcal{X}\right|_{s} \cong \mathcal{C}_{V_{\beta}}\right|_{u}$ given.

We first introduce $\mathbf{Q}$-line bundles on $\mathcal{M}_{g, m}$. We fix an integer $k \geq 0$ and let $m=n+k$. For any

$$
K=\left\{h_{1}, \cdots, h_{k_{1}}\right\} \subset \Sigma \triangleq\{n+1, \cdots, m\}
$$

we let $K^{\prime}=\left\{h_{1}^{\prime}, \cdots, h_{k_{2}}^{\prime}\right\}$ be the complement of $K$. Here we assume that both $h_{i}$ and $h_{j}^{\prime}$ are strictly increasing. We call $K$ and $K^{\prime}$ a partition of $\Sigma$. Given any $K \subset \Sigma$, there is an obvious clutching transformation

$$
\tilde{\tau}_{K}: \mathfrak{F}_{g_{1}, m_{1}+1} \times \mathfrak{F}_{g_{2}, m_{2}+1} \longrightarrow \mathfrak{F}_{g, n},
$$

where $m_{i}=n_{i}+k_{i}$. Namely, given $C_{1} \in \mathfrak{F}_{g_{1}, m_{1}+1}(S)$ and $C_{2} \in \mathfrak{F}_{g_{2}, m_{2}+1}(S)$, we let $\tilde{\tau}_{K}\left(C_{1}, C_{2}\right)$ be the family obtained by identifying the last section in $C_{1}$ with the first section in $C_{2}$, and set the $m$ marked sections to be the union of all but the last sections of $C_{1}$ and all but the first sections of $C_{2}$. They are ordered as follows. The first $n$ sections are $s_{1}^{1}, \cdots, s_{n_{1}}^{1}, s_{2}^{2}, \cdots, s_{n_{2}+1}^{2}$, where $\left\{s_{i}^{1}\right\}$ and $\left\{s_{j}^{2}\right\}$ are sections of $C_{1}$ and $C_{2}$ respectively. We then place $s_{n_{1}+i}^{1}$ in the $h_{i}$-th place and place $s_{n_{2}+j+1}^{2}$ in the $h_{j}^{\prime}$-th place. We denote by $d: S \rightarrow C$ the section of nodal points along which the gluing is taking place. We call such a nodal section a decomposable nodal section, and say $C$ is decomposable into families in $\mathfrak{F}_{g_{1}, m_{1}+1}$ and $\mathfrak{F}_{g_{2}, m_{2}+1}$ along the partition $K \cup K^{\prime}$ (or along the nodal section $d$ ).

Since $n_{1}, n_{2}>0, \tau_{K}$ is a closed immersion [Kn], the image scheme of $\tau_{K}$ is a Q-Cartier divisor. Let $\left\{\left(U_{\beta}, U_{\beta} / G_{\beta}, C_{U_{\beta}}\right)\right\}_{\beta \in \Lambda_{m}}$ be an atlas of $\mathcal{M}_{g, m}$. For each $\beta \in \Lambda_{m}$, there is an $f_{K, \beta} \in \mathcal{O}_{U_{\beta}}$ such that $T_{K, \beta}=\left\{f_{K, \beta}=0\right\}$ is exactly the subscheme such that the restriction of $C_{U_{\beta}}$ to it is decomposable into families in $\mathfrak{F}_{g_{1}, m_{1}+1}$ and $\mathfrak{F}_{g_{2}, m_{2}+1}$ along the partition $K \cup K^{\prime}$ (see [DM, p. 83]). If $T_{K, \beta}=\emptyset$, 
we will set $f_{K, \beta} \equiv 1$. Note that in doing so, we might need to shrink $U_{\beta}$ if necessary. (In the following, we shall feel free to shrink $U_{\beta}$ and make necessary adjustments whenever necessary.) By the theory of deformation of nodal points, $\left.d f_{K, \beta}\right|_{T_{K, \beta}}$ is nowhere vanishing. Furthermore, over $U_{\beta \beta^{\prime}}$, there is an $f_{K, \beta \beta^{\prime}} \in \mathcal{O}_{U_{\beta \beta^{\prime}}}^{*}$ such that

$$
f_{K, \beta} \circ \pi_{U_{\beta}}=f_{K, \beta \beta^{\prime}} \cdot\left(f_{K, \beta^{\prime}} \circ \pi_{U_{\beta^{\prime}}}\right),
$$

where as usual we denote by $\pi_{U_{\beta}}: U_{\beta \beta^{\prime}} \rightarrow U_{\beta}$ the projection. Clearly, $\left\{f_{K, \beta \beta^{\prime}}\right\}$ satisfies the cocycle condition over the triple overlaps. Hence $\left\{f_{K, \beta \beta^{\prime}}\right\}$ defines a Q-line bundle, denoted by $L_{K}$, and $\left\{f_{K, \beta}\right\}$ defines a section $f_{K}$ of $L_{K}$. By our choice of $f_{K, \beta}$, the image $\mathbf{Q}$-scheme $\operatorname{Im}\left(\tau_{K}\right)$ is defined in each $U_{\beta}$ by the vanishing of $f_{K, \beta}$.

We now turn our attention to the contraction transformation $\tilde{\pi}_{n}^{m}: \mathfrak{F}_{g, m} \longrightarrow \mathfrak{F}_{g, n}$ that sends any curve to the curve obtained by first forgetting the last $k$ sections of the curve and then stable contracting the resulting family. We denote by $\pi_{n}^{m}$ the morphism between the corresponding moduli schemes. For each $\beta \in \Lambda_{m}$, there is an étale covering $\pi_{j}: A_{j} \rightarrow U_{\beta}$ of $U_{\beta}$ and $\beta_{j}^{\prime} \in \Lambda_{n}$ such that there is a morphism $\pi_{n, \beta, j}^{m}: A_{j} \rightarrow V_{\beta_{j}^{\prime}}$ induced by the transformation $\tilde{\pi}_{n}^{m}$. For simplicity, after replacing $U_{\beta}$ by an étale covering of itself and rearranging the index, we can assume that $\Lambda_{n}=\Lambda_{m} \triangleq \Lambda$ and that the maps $\pi_{n, \beta, j}^{m}$ just mentioned are from $U_{\beta}$ to $V_{\beta}$, denoted $\pi_{n, \beta}^{m}$ (we allow some $U_{\beta}$ or $V_{\beta}$ to be empty sets). Clearly, $\pi_{n, \beta}^{m}$ is $G_{\beta}$-equivariant, under the obvious group homomorphism $G_{\beta} \rightarrow H_{\beta}$. Let $f_{n, \beta} \in \mathcal{O}_{V_{\beta}}$ be a section whose vanishing locus defines the closed subscheme $T_{n, \beta} \subset V_{\beta}$, where $T_{n, \beta}$ is the largest subscheme over which the family $\mathcal{C}_{V_{\beta}}$ decomposes into families in $\mathfrak{F}_{g_{1}, n_{1}+1}$ and $\mathfrak{F}_{g_{2}, n_{2}+1}$. Let

$$
f_{n, \beta \beta^{\prime}}=\left(f_{n, \beta} \circ \pi_{V_{\beta}}\right) /\left(f_{n, \beta^{\prime}} \circ \pi_{V_{\beta^{\prime}}}\right),
$$

where $\pi_{V_{\beta}}: V_{\beta \beta^{\prime}} \rightarrow V_{\beta}$ are the projections. $\left\{f_{n, \beta \beta^{\prime}}\right\}$ defines a $\mathbf{Q}$-line bundle $L_{n}$ on $\mathcal{M}_{g, n}$ and $\left\{f_{n, \beta}\right\}$ is a global $\mathbf{Q}$-section of $L_{n}$ whose vanishing locus defines the image $\mathbf{Q}$-scheme of the clutching morphism

$$
\tau_{g_{\bullet} n_{\bullet}}: \mathcal{M}_{g_{1}, n_{1}+1} \times \mathcal{M}_{g_{2}, n_{2}+1} \longrightarrow \mathcal{M}_{g, n} .
$$

By [DM, p. 83], there is a nowhere vanishing $g_{\beta} \in \mathcal{O}_{U_{\beta}}^{*}$ such that

$$
\prod_{K \subset \Sigma} f_{K, \beta}=g_{\beta} \cdot\left(f_{n, \beta} \circ \pi_{n, \beta}^{m}\right) .
$$

By replacing one $f_{K, \beta}$ by $f_{K, \beta} / g_{\beta}$, we can assume that $g_{\beta} \equiv 1$. Then

$$
\bigotimes_{K \subset \Sigma} L_{K}=\left(\pi_{n}^{m}\right)^{*} L_{n} \quad \text { and } \prod_{K \subset \Sigma} f_{K}=\left(\pi_{n}^{m}\right)^{*}\left(f_{n}\right) .
$$

In the next part, for any partition $\alpha_{1}+\alpha_{2}=\alpha$ we will construct the $\mathbf{Q}$-line bundle $L_{\alpha_{1} \alpha_{2}}$ on $\mathcal{M}_{\alpha, g, n}^{X}$ and a section $f_{\alpha_{1} \alpha_{2}}$ of $L_{\alpha_{1} \alpha_{2}}$ such that $f_{\alpha_{1} \alpha_{2}}=0$ defines the image $\mathbf{Q}$-scheme of the clutching morphism $\tau_{\alpha_{1} \alpha_{2}}$. We fix a sufficiently large $m$. Let $\beta \in \Lambda_{\alpha}$ and let $\left(W_{\beta}, K_{\beta}, F_{W_{\beta}}, \mathcal{C}_{W_{\beta}}\right)$ be the chart in the atlas of $\mathcal{M}_{\alpha, g, n}^{X}$. Without loss of generality, we can assume that there are sections $s_{n+1, \beta}, \cdots, s_{m, \beta}$ : $W_{\beta} \rightarrow \mathcal{C}_{W_{\beta}}$ such that $\mathcal{C}_{W_{\beta}}$ with these extra sections is a family of $m$-pointed stable curves. We fix such a choice of new sections for each $\beta$ once and for all. We denote the resulting $m$-pointed curve by $\overline{\mathcal{C}}_{W_{\beta}}$. As we did before, after rearrangement we can assume that $\Lambda_{\alpha}=\Lambda$ and that for each $\beta \in \Lambda$ there is a morphism $\pi_{m, \beta}^{\alpha}: W_{\beta} \rightarrow U_{\beta}$ 
such that the pull back of $\mathcal{C}_{U_{\beta}}$ is isomorphic to $\overline{\mathcal{C}}_{W_{\beta}}$. We fix such $\pi_{m, \beta}^{\alpha}$ once and for all.

Now let $\beta \in \Lambda$ be an index. We assume that for some partition $\alpha_{1}+\alpha_{2}=\alpha$, $p_{\beta}^{-1}\left(\operatorname{Im}\left(\tau_{\alpha_{1} \alpha_{2}}\right)\right) \neq \emptyset$, where $p_{\beta}: W_{\beta} \rightarrow \mathcal{M}_{\alpha, g, n}^{X}$ is the classifying map. Let $w$ be a point in this set. Then there is a unique partition $K \cup K^{\prime}=\Sigma$ such that the curve associated to $w$ with the extra sections is in the image of the clutching transformation $\tau_{K}$. Without loss of generality, we can assume that the partitions $K \cup K^{\prime}$ associated to $w \in p_{\beta}^{-1}\left(\operatorname{Im}\left(\tau_{\alpha_{1} \alpha_{2}}\right)\right)$ are independent of $w$. Then the subscheme

$$
T_{\alpha_{1} \alpha_{2}, \beta}=\left\{f_{K, \beta} \circ \pi_{m, \beta}^{\alpha}=0\right\} \subset W_{\beta}
$$

is the closed subscheme such that the restriction of the family $F_{W_{\beta}}$ to $T_{\alpha_{1} \alpha_{2}, \beta}$ is in the image of the clutching transformation

$$
\tilde{\tau}_{\alpha_{1} \alpha_{2}}: \mathfrak{F}_{\alpha_{1}, g_{1}, n_{1}+1}^{X} \times \mathfrak{F}_{\alpha_{2}, g_{2}, n_{2}+1}^{X} \longrightarrow \mathfrak{F}_{\alpha, g, n}^{X} .
$$

(Here we might need to shrink $W_{\beta}$ so that any element in $\left\{f_{K, \beta} \circ \pi_{m, \beta}^{\alpha}=0\right\}$ is over the image of $\tau_{\alpha_{1} \alpha_{2}}$.) We let $f_{\alpha_{1} \alpha_{2}, \beta}=f_{K, \beta} \circ \pi_{m, \beta}^{\alpha}$. In case $p_{\beta}^{-1}\left(\operatorname{Im}\left(\tau_{\alpha_{1} \alpha_{2}}\right)\right)=\emptyset$, we set $f_{\alpha_{1} \alpha_{2}, \beta} \equiv 1$. Clearly, should there be a unique $f_{\alpha_{1} \alpha_{2}, \beta \beta^{\prime}} \in \mathcal{O}_{W_{\beta \beta^{\prime}}}^{*}$ such that

$$
f_{\alpha_{1} \alpha_{2}, \beta} \circ \pi_{W_{\beta}}=f_{\alpha_{1} \alpha_{2}, \beta \beta^{\prime}} \cdot\left(f_{\alpha_{1} \alpha_{2}, \beta^{\prime}} \circ \pi_{W_{\beta^{\prime}}}\right)
$$

for all pairs $\left\{\beta, \beta^{\prime}\right\}$, where $\pi_{W_{\beta}}: W_{\beta \beta^{\prime}} \rightarrow W_{\beta}$ is the projection, then $\left\{f_{\alpha_{1} \alpha_{2}, \beta \beta^{\prime}}\right\}$ would satisfy the desired cocycle condition and then define a $\mathbf{Q}$-line bundle, and $\left\{f_{\alpha_{1} \alpha_{2}, \beta}\right\}$ would define a global section whose vanishing locus is the image of $\tau_{\alpha_{1} \alpha_{2}}$, as desired. However, for our moduli space $\mathcal{M}_{\alpha, g, n}^{X}$, it may happen that some $f_{\alpha_{1} \alpha_{2}, \beta} \equiv 0$. Thus the above direct argument needs to be modified. In the following, we will first thicken the $W_{\beta}$ 's so that the desired $f_{\alpha_{1} \alpha_{2}, \beta \beta^{\prime}}$ are still well-defined.

We fix a two-term complex $\mathcal{E}^{\bullet}$ of locally free sheaves such that its sheaf cohomology $\mathfrak{H}^{\bullet}\left(\mathcal{E}^{\bullet}\right)=\mathcal{T} \bullet \mathfrak{F}_{\alpha, g, n}^{X}$. Such complexes were constructed in section 4 . Over each $W_{\beta}, \mathcal{T}^{\bullet} \mathfrak{F}_{\alpha, g, n}^{X}$ is represented by a tangent-obstruction complex $\mathcal{T}_{W_{\beta}}$ of $W_{\beta}$, and $\mathcal{E}^{\bullet}$ is represented by a two-term complex of locally free sheaves of $\mathcal{O}_{\mathcal{W}_{\beta}}$-modules, denoted $\mathcal{E}_{\beta}^{\bullet}=\left[\mathcal{E}_{\beta, 1} \rightarrow \mathcal{E}_{\beta, 2}\right]$, such that $\mathfrak{H}^{\bullet}\left(\mathcal{E}_{\beta}^{\bullet}\right)=\mathcal{T}_{W_{\beta}}^{\bullet}$. Let $E_{\beta}$ be the vector bundle on $W_{\beta}$ such that $\mathcal{O}_{W_{\beta}}\left(E_{\beta}\right)=\mathcal{E}_{\beta, 1}$. By abuse of notation, we will also use $E_{\beta}$ to denote the total space of $E_{\beta}$. Because of the way $\mathcal{E}^{\bullet}$ is constructed, there is a canonical homomorphism of locally free sheaves

$$
\varphi_{\beta}: \mathcal{E}_{\beta, 1} \longrightarrow \mathcal{E} t_{\mathcal{C}_{W_{\beta}} / W_{\beta}}^{1}\left(\Omega_{\mathcal{C}_{W_{\beta}} / W_{\beta}}\left(D_{W_{\beta}}\right), \mathcal{O}_{\mathcal{C}_{W_{\beta}}}\right),
$$

where $D_{W_{\beta}}$ is the divisor of $n$-marked sections of $\mathcal{C}_{W_{\beta}}$. By the deformation theory, if we let $J \subset \mathcal{O}_{E_{\beta}}$ be the ideal sheaf of $W_{\beta} \subset E_{\beta}$, embedded via the zero section, and let $F_{\beta} \subset E_{\beta}$ be the subscheme defined by the ideal $J^{2}$, then there is a family of $n$-pointed curves $\mathcal{C}_{F_{\beta}}$ over $F_{\beta}$ such that the homomorphism $\varphi_{\beta}$ above is the Kodaira-Spencer map of the family $\mathcal{C}_{F_{\beta}}$ along the normal bundle to $W_{\beta}$ in $F_{\beta}$. Here by the normal bundle to $W_{\beta}$ in $F_{\beta}$ we mean the subbundle of $\left.T F_{\beta}\right|_{W_{\beta}}$ that is the kernel of $\left.T F_{\beta}\right|_{W_{\beta}} \rightarrow T W_{\beta}$. Since the restriction of $\mathcal{C}_{F_{\beta}}$ to $W_{\beta} \subset F_{\beta}$ is $\mathcal{C}_{W_{\beta}}$ and $\overline{\mathcal{C}}_{W_{\beta}}$ is smooth over $W_{\beta}$ near its sections, we can extend the last $k$ sections of $\overline{\mathcal{C}}_{W_{\beta}}$ to sections in $\mathcal{C}_{F_{\beta}}$ (over $F_{\beta}$ ) and place them in the same order as $\overline{\mathcal{C}}_{\mathcal{W}_{\beta}}$ has. We denote the resulting $m$-pointed curve by $\overline{\mathcal{C}}_{F_{\beta}}$, which is a stable curve over $F_{\beta}$. Let

$$
\bar{\pi}_{m, \beta}^{\alpha}: F_{\beta} \rightarrow U_{\beta}
$$

be the morphism induced by the family $\overline{\mathcal{C}}_{F_{\beta}}$. Then $\bar{\pi}_{m, \beta}^{\alpha}$ is an extension of $\pi_{m, \beta}^{\alpha}$ : $W_{\beta} \rightarrow U_{\beta}$. 
Now we go back to the partition $K \cup K^{\prime}=\Sigma$ and the $T_{\alpha_{1} \alpha_{2}, \beta}$ defined in (5.1). We let $\bar{f}_{\alpha_{1} \alpha_{2}, \beta}$ be $f_{K, \beta} \circ \bar{\pi}_{m, \beta}^{\alpha} \in \mathcal{O}_{F_{\beta}}$. Then $\bar{T}=\left\{\bar{f}_{\alpha_{1} \alpha_{2}, \beta}=0\right\}$ is exactly the subscheme of $F_{\beta}$ such that the restriction of $\overline{\mathcal{C}}_{F_{\beta}}$ to $\bar{T}$ belongs to the image of the clutching transformation

$$
\tilde{\pi}_{K}: \mathfrak{F}_{\alpha_{1}, g_{1}, m_{1}+1}^{X} \times \mathfrak{F}_{\alpha_{2}, g_{2}, m_{2}+1}^{X} \longrightarrow \mathfrak{F}_{\alpha, g, n}^{X} .
$$

We fix such a $\overline{\mathcal{C}}_{F_{\beta}}$, and then $\bar{\pi}_{m, \beta}^{\alpha}$ and $\bar{f}_{\alpha_{1} \alpha_{2}, \beta}$ for each $\beta \in \Lambda$ once and for all. Recall that $\pi_{W_{\beta}}: W_{\beta \beta^{\prime}} \rightarrow W_{\beta}$ is the projection. Since there is a canonical isomorphism

$$
\left(\pi_{W_{\beta}}\right)^{*} E_{\beta} \cong\left(\pi_{W_{\beta^{\prime}}}\right)^{*} E_{\beta^{\prime}}
$$

$F_{\beta} \times_{W_{\beta}} W_{\beta \beta^{\prime}}$ is canonically isomorphic to $F_{\beta^{\prime}} \times_{W_{\beta^{\prime}}} W_{\beta \beta^{\prime}}$. We denote this scheme by $F_{\beta \beta^{\prime}}$. We let $\pi_{F_{\beta}}: F_{\beta \beta^{\prime}} \rightarrow F_{\beta}$ be the projection.

Lemma 5.5. There are $\phi \in \mathcal{O}_{F_{\beta \beta^{\prime}}}$ such that

$$
\bar{f}_{\alpha_{1} \alpha_{2}, \beta} \circ \pi_{F_{\beta}}=\phi \cdot\left(\bar{f}_{\alpha_{1} \alpha_{2}, \beta^{\prime}} \circ \pi_{F_{\beta^{\prime}}}\right) .
$$

Further, if we let $\iota: W_{\beta \beta^{\prime}} \rightarrow F_{\beta \beta^{\prime}}$ be the inclusion, then $\phi \circ \iota$ is unique and is nowhere vanishing. We denote $\phi \circ \iota$ by $f_{\alpha_{1} \alpha_{2}, \beta \beta^{\prime}}$. $\left\{f_{\alpha_{1} \alpha_{2}, \beta \beta^{\prime}}\right\}$ defines a $\mathbf{Q}$-line bundle on $\mathcal{M}_{\alpha, g, n}^{X}$ and $\left\{f_{\alpha_{1} \alpha_{2}, \beta}\right\}$ defines a section of this line bundle.

Proof. The existence of $\phi$ follows from the fact that the subscheme $\left\{\bar{f}_{\alpha_{1} \alpha_{2}, \beta} \circ \pi_{F_{\beta}}=\right.$ $0\}$ is identical to the subscheme $\left\{\bar{f}_{\alpha_{1} \alpha_{2}, \beta^{\prime}} \circ \pi_{F_{\beta^{\prime}}}=0\right\}$. This is true because both define the subschemes over which the two families of curves, which are isomorphic after discarding the last $k$ sections, can be decomposed along the same nodal sections (see [DM, p. 83]). To show that $\phi \circ \iota$ is unique, it suffices to show that at each $w \in W_{\beta \beta^{\prime}}$ there is a tangent vector $v \in T_{w} F_{\beta \beta^{\prime}}$ contained in the kernel of $T_{w} F_{\beta \beta^{\prime}} \rightarrow T_{w} W_{\beta \beta^{\prime}}$ such that $d\left(\bar{f}_{\alpha_{1} \alpha_{2}, \beta}\right)(v) \neq 0$. Here $T_{w} F_{\beta \beta^{\prime}} \rightarrow T_{w} W_{\beta \beta^{\prime}}$ is induced by the vector bundle projection $E_{\beta} \rightarrow W_{\beta}$. This is true because

$$
\mathcal{E}_{\beta, 1} \otimes k(w) \longrightarrow \operatorname{Ext}_{\mathcal{C}_{w}}^{1}\left(\Omega_{\mathcal{C}_{w}}\left(D_{w}\right), \mathcal{O}_{\mathcal{C}_{w}}\right) \longrightarrow \mathcal{E} x t_{\hat{r}}^{1}\left(\Omega_{\hat{r}}, \mathcal{O}_{\hat{r}}\right)
$$

is surjective. Here $\mathcal{C}_{w}$ is the fiber of $\mathcal{C}_{U_{\beta}}$ over $w, D_{w} \subset \mathcal{C}_{w}$ is the divisor of $n$-marked points, $r \in \mathcal{C}_{w}$ is the nodal point where the clutching $\tau_{\alpha_{1} \alpha_{2}}$ is taking place and $\hat{r}$ is the formal completion of $\mathcal{C}_{w}$ along $r$. Because $f_{\alpha_{1} \alpha_{2}, \beta \beta^{\prime}}$ are unique, the collection $\left\{f_{\alpha_{1} \alpha_{2}, \beta \beta^{\prime}}\right\}$ satisfies the cocycle condition on triple overlaps. Therefore it defines a $\mathbf{Q}$-line bundle on $\mathcal{M}_{\alpha, g, n}^{X}$, denoted by $L_{\alpha_{1} \alpha_{2}}$. For the same reason, $\left\{f_{\alpha_{1} \alpha_{2}, \beta}\right\}$ forms a global section, denoted by $f_{\alpha_{1} \alpha_{2}}$, of $L_{\alpha_{1} \alpha_{2}}$ such that the image scheme $\operatorname{Im}\left(\tau_{\alpha_{1} \alpha_{2}}\right) \subset \mathcal{M}_{\alpha, g, n}^{X}$ is defined over each $W_{\beta}$ by $\left\{f_{\alpha_{1} \alpha_{2}, \beta}=0\right\}$. This proves the lemma.

Now, we let $\pi_{n}^{\alpha}: \mathcal{M}_{\alpha, g, n}^{X} \rightarrow \mathcal{M}_{g, n}$ be the stable contraction morphism. Locally, $\pi_{n}^{\alpha}$ is represented by maps

$$
\pi_{n, \beta}^{\alpha}=\pi_{n, \beta}^{m} \circ \pi_{m, \beta}^{\alpha}: W_{\beta} \longrightarrow V_{\beta} .
$$

Let $\pi_{n, \beta \beta^{\prime}}^{\alpha}: W_{\beta \beta^{\prime}} \rightarrow V_{\beta \beta^{\prime}}$ be the lifting of the pair $\left(\pi_{n, \beta}^{\alpha}, \pi_{n, \beta^{\prime}}^{\alpha}\right)$, which exists and is unique. Let $f_{\alpha, \beta} \in \mathcal{O}_{W_{\beta}}$ be $f_{n, \beta} \circ \pi_{n, \beta}^{\alpha}$ and let $f_{\alpha, \beta \beta^{\prime}}=f_{n, \beta \beta^{\prime}} \circ \pi_{n, \beta \beta^{\prime}}^{\alpha} \in \mathcal{O}_{W_{\beta \beta^{\prime}}}^{*}$. Then $\left\{f_{\alpha, \beta \beta^{\prime}}\right\}$ defines a $\mathbf{Q}$-line bundle, denoted by $L_{\alpha}$, which is isomorphic to the pull back $\left(\pi_{n}^{\alpha}\right)^{*} L_{n}$. For the same reason, $\left\{f_{\alpha, \beta}\right\}$ is a section $f_{\alpha}$ of $L_{\alpha}$, which is the pull back of $f_{n}$ of $L_{n}$. 
Lemma 5.6. There is a canonical isomorphism of $\mathbf{Q}$-line bundles $\otimes L_{\alpha_{1} \alpha_{2}} \cong L_{\alpha}$ such that under this isomorphism, we have $\prod f_{\alpha_{1} \alpha_{2}}=f_{\alpha}$, where the product is over all possible $\alpha_{1}+\alpha_{2}=\alpha$.

Proof. First note that given any $\alpha_{1}+\alpha_{2}=\alpha$ and $w \in p_{\beta}^{-1}\left(\operatorname{Im}\left(\tau_{\alpha_{1} \alpha_{2}}\right)\right)$, there is a unique partition $K \cup K^{\prime}=\Sigma$ such that the decomposition of $\overline{\mathcal{C}}_{w}$ along the partition $K \cup K^{\prime}$ is the inverse to the clutching transformation $\tilde{\tau}_{\alpha_{1} \alpha_{2}}$. We denote this correspondence by $\alpha_{1} \alpha_{2} \mapsto K_{w}\left(\alpha_{1} \alpha_{2}\right)$. By making each $W_{\beta}$ small enough, we can assume that the following two conditions hold automatically for all $\beta \in \Lambda$. First, for $w, w^{\prime} \in p_{\beta}^{-1}\left(\operatorname{Im}\left(\tau_{\alpha_{1} \alpha_{2}}\right)\right)$ we have $K_{w}\left(\alpha_{1} \alpha_{2}\right)=K_{w^{\prime}}\left(\alpha_{1} \alpha_{2}\right)$; second, the map

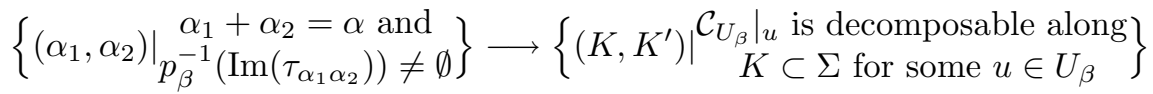

is injective and onto. The first is possible because $\tau_{\alpha_{1} \alpha_{2}}$ is a closed embedding. We define $K_{\beta}\left(\alpha_{1} \alpha_{2}\right)=K_{w}\left(\alpha_{1} \alpha_{2}\right)$ for some $w \in p_{\beta}^{-1}\left(\operatorname{Im}\left(\tau_{\alpha_{1} \alpha_{2}}\right)\right)$. Hence for each $\beta$,

$$
\prod_{K \subset \Sigma} f_{K, \beta}=f_{n, \beta} \circ \pi_{n, \beta}^{m} .
$$

Here if $K \cup K^{\prime}$ is not in the image of the above correspondence, then $f_{K, \beta} \equiv 1$. To show that $\otimes L_{\alpha_{1} \alpha_{2}}=L_{\alpha}$, we need to check $\prod f_{\alpha_{1} \alpha_{2}, \beta \beta^{\prime}}=f_{\alpha, \beta \beta^{\prime}}$, which amounts to proving the identity

$$
\prod_{\alpha_{1}+\alpha_{2}=\alpha} \frac{\left(\pi_{m, \beta}^{\alpha} \circ \pi_{W_{\beta}}\right)^{*} f_{K_{\beta}\left(\alpha_{1} \alpha_{2}\right), \beta}}{\left(\pi_{m, \beta^{\prime}}^{\alpha} \circ \pi_{W_{\beta^{\prime}}}\right)^{*} f_{K_{\beta^{\prime}}\left(\alpha_{1} \alpha_{2}\right), \beta^{\prime}}}=\frac{\left(\pi_{n, \beta}^{\alpha} \circ \pi_{W_{\beta}}\right)^{*} f_{n, \beta}}{\left(\pi_{n, \beta^{\prime}}^{\alpha} \circ \pi_{W_{\beta^{\prime}}}\right)^{*} f_{n, \beta^{\prime}}},
$$

on $W_{\beta \beta^{\prime}}$. This is obvious from the previous identity if all quotients are well-defined and unique. To prove (5.3), we first embed $F_{\beta \beta^{\prime}} \supset W_{\beta \beta^{\prime}}$ into a smooth affine scheme, say $F_{\beta \beta^{\prime}} \subset R$. Without loss of generality, we can assume $W_{\beta}, U_{\beta}$ and $W_{\beta \beta^{\prime}}$ are affine. Let $\phi: R \rightarrow U_{\beta}$ be a morphism extending the morphism

$$
\psi_{\beta}: F_{\beta \beta^{\prime}} \stackrel{\pi_{F_{\beta}}}{\longrightarrow} F_{\beta} \stackrel{\bar{\pi}_{m, \beta}^{\alpha}}{\longrightarrow} U_{\beta} .
$$

We claim that there is a morphism $\phi^{\prime}: R \rightarrow U_{\beta^{\prime}}$ extending $\psi_{\beta^{\prime}}: F_{\beta \beta^{\prime}} \rightarrow U_{\beta^{\prime}}$ such that

$$
p_{\beta} \circ \pi_{n, \beta}^{m} \circ \phi=p_{\beta^{\prime}} \circ \pi_{n, \beta^{\prime}}^{m} \circ \phi^{\prime},
$$

where $p_{\beta}: V_{\beta} \rightarrow \mathcal{M}_{g, n}$ is the obvious morphism. Indeed, the morphism $\phi: R \rightarrow U_{\beta}$ provides a family of $m$-pointed stable curves over $R$ via pull back. Let it be $\mathcal{X}_{R}$ with the sections $\left\{s_{i}\right\}$. Let $\psi_{\beta^{\prime}}^{*} \mathcal{C}_{U_{\beta^{\prime}}}$ be the pull back of $\mathcal{C}_{U_{\beta^{\prime}}}$ via $\psi_{\beta^{\prime}}: F_{\beta \beta^{\prime}} \rightarrow U_{\beta^{\prime}}$. Then after restricting $\mathcal{X}_{R}$ to $F_{\beta \beta^{\prime}}$ and discarding its last $k$ sections, the resulting family is isomorphic to $\psi_{\beta^{\prime}}^{*} \mathcal{C}_{U_{\beta^{\prime}}}$ as $n$-pointed curves. We choose $k$ sections $\tilde{s}_{n+1}, \cdots, \tilde{s}_{m}$ of $\mathcal{X}_{R}$ extending the last $k$ sections of $\psi_{\beta^{\prime}}^{*} \mathcal{C}_{\beta^{\prime}}$, which is possible because $\mathcal{X}_{R}$ is smooth over $R$ along sections of $\psi_{\beta^{\prime}}^{*} \mathcal{C}_{\beta^{\prime}}$. We denote $\mathcal{X}_{R}$ with these new sections $\left(\cdots, s_{n}, \tilde{s}_{n+1}, \cdots\right)$ by $\tilde{\mathcal{X}}_{R}$. Then $\tilde{\mathcal{X}}_{R}$ induces a morphism $\phi^{\prime}: R \rightarrow U_{\beta^{\prime}}$ that has the desired property.

Now we show that $\prod f_{\alpha_{1} \alpha_{2}, \beta \beta^{\prime}}=f_{\alpha, \beta \beta^{\prime}}$. Because of (5.4) above and because $\phi^{\prime}$ is induced by the family of curves, we have a unique lifting $\bar{\phi}: R \rightarrow V_{\beta \beta^{\prime}}$. Then because $R$ is smooth and because neither $f_{K_{\beta}\left(\alpha_{1} \alpha_{2}\right), \beta} \circ \phi$ nor $f_{K_{\beta^{\prime}}\left(\alpha_{1} \alpha_{2}\right), \beta^{\prime}} \circ \phi^{\prime}$ is zero, there is a unique rational $h_{\alpha_{1} \alpha_{2}, \beta \beta^{\prime}}$ such that

$$
f_{K_{\beta}\left(\alpha_{1} \alpha_{2}\right), \beta} \circ \phi=h_{\alpha_{1} \alpha_{2}, \beta \beta^{\prime}} \cdot\left(f_{K_{\beta^{\prime}}\left(\alpha_{1} \alpha_{2}\right), \beta^{\prime}} \circ \phi^{\prime}\right) \text {. }
$$


Clearly, $h_{\alpha_{1} \alpha_{2}, \beta \beta^{\prime}}$ is regular and non-vanishing near $F_{\beta \beta^{\prime}} \subset R$. It follows that

$$
\prod_{\alpha_{1}+\alpha_{2}=\alpha}\left(f_{K_{\beta}\left(\alpha_{1} \alpha_{2}\right), \beta} \circ \phi\right)=\left(\prod_{\alpha_{1}+\alpha_{2}=\alpha} h_{\alpha_{1} \alpha_{2}, \beta \beta^{\prime}}\right)\left(\prod_{\alpha_{1}+\alpha_{2}=\alpha}\left(f_{K_{\beta^{\prime}}\left(\alpha_{1} \alpha_{2}\right), \beta^{\prime}} \circ \phi^{\prime}\right)\right) .
$$

Now let $\tau_{\beta \beta^{\prime}}: W_{\beta \beta^{\prime}} \rightarrow R$ be the inclusion. Of course, $h_{\alpha_{1} \alpha_{2}, \beta \beta^{\prime}} \circ \tau_{\beta \beta^{\prime}} \equiv f_{\alpha_{1} \alpha_{2}, \beta \beta^{\prime}}$. Thus to show that $\otimes L_{\alpha_{1} \alpha_{2}}=\left(\pi_{n}^{\alpha}\right)^{*} L_{n}$, it suffices to show that

$$
\left(\prod h_{\alpha_{1} \alpha_{2}, \beta \beta^{\prime}}\right) \circ \tau_{\beta \beta^{\prime}}=f_{n, \beta \beta^{\prime}} \circ \pi_{n, \beta \beta^{\prime}}^{\alpha},
$$

where $\pi_{n, \beta \beta^{\prime}}^{\alpha}: W_{\beta \beta^{\prime}} \rightarrow V_{\beta \beta^{\prime}}$ is the projection. Indeed, because $\prod_{K \subset \Sigma} f_{K, \beta}=$ $f_{n, \beta} \circ \pi_{n, \beta}^{m, \beta}$,

$$
g_{\beta}:=f_{n, \beta} \circ \pi_{n, \beta}^{m} \circ \phi=\prod_{\alpha_{1}+\alpha_{2}=\alpha} f_{K_{\beta}\left(\alpha_{1} \alpha_{2}\right), \beta} \circ \phi .
$$

Therefore (5.5) holds with $\prod h_{\alpha_{1} \alpha_{2}, \beta \beta^{\prime}}$ replaced by $g_{\beta} / g_{\beta^{\prime}}$. Since all terms in (5.5) are non-trivial and since $R$ is smooth, $g_{\beta} / g_{\beta^{\prime}}$ is regular near $W_{\beta \beta^{\prime}}$ and its composite with $\tau_{\beta \beta^{\prime}}$ is identical to the composite of $\prod h_{\alpha_{1} \alpha_{2}, \beta \beta^{\prime}}$ with $\tau_{\beta \beta^{\prime}}$. Finally, because this term is also the pull back via $\bar{\phi}: R \rightarrow V_{\beta \beta^{\prime}}$ of $f_{n, \beta} \circ \pi_{V_{\beta}} / f_{n, \beta^{\prime}} \circ \pi_{V_{\beta^{\prime}}}$, where $\pi_{V_{\beta}}$ : $V_{\beta \beta^{\prime}} \rightarrow V_{\beta}$ is the projection, its composite with $\tau_{\beta \beta^{\prime}}$ is the pull back of $f_{n, \beta \beta^{\prime}}$. This proves that $\prod f_{\alpha_{1} \alpha_{2}, \beta \beta^{\prime}}=f_{\alpha, \beta \beta^{\prime}}$ and consequently $\otimes L_{\alpha_{1} \alpha_{2}}=\left(\pi_{n}^{\alpha}\right)^{*} L_{n}$. It remains to show that $\prod f_{\alpha_{1} \alpha_{2}, \beta}=f_{\alpha, \beta}$. This is true because $\prod_{K \subset \Sigma} f_{K, \beta}=f_{n, \beta} \circ \pi_{n, \beta}^{m}$. This completes the proof of Lemma 5.5.

As was explained before, to complete the proof of the first composition law, we remain to investigate the tangent-obstruction complex of $Z_{\alpha_{1} \alpha_{2}}$. We first introduce the functor $\mathfrak{F}_{\alpha_{1} \alpha_{2}}$ so that its coarse moduli scheme is $Z_{\alpha_{1} \alpha_{2}}$. For any scheme $S$, we let $\mathfrak{F}_{\alpha_{1} \alpha_{2}}(S)$ be the subset of $\mathfrak{F}_{\alpha, g, n}^{X}(S)$ consisting of families $f: \mathcal{X} \rightarrow X$ such that there are distinguished sections of nodal points $d: S \rightarrow \mathcal{X}$ such that $f$ are decomposable to pairs of families in $\mathfrak{F}_{\alpha_{1}, g_{1}, n_{1}+1}^{X}(S) \times \mathfrak{F}_{\alpha_{2}, g_{2} n_{2}+1}^{X}(S)$ along the nodal sections $d$. Clearly, $\mathfrak{F}_{\alpha_{1} \alpha_{2}}$ is coarsely represented by a scheme $V_{\alpha_{1} \alpha_{2}}$ which is canonically isomorphic to the $Z_{\alpha_{1} \alpha_{2}}$ defined before. By forgetting the distinguished sections, the resulting transformation $\mathfrak{F}_{\alpha_{1} \alpha_{2}} \rightarrow \mathfrak{F}_{\alpha, g, n}^{X}$ defines a morphism $\Phi: Z_{\alpha_{1} \alpha_{2}} \rightarrow \mathcal{M}_{\alpha, g, n}^{X} . \Phi$ is a closed immersion since $n_{1}, n_{2}>0$ and induces an isomorphism $Z_{\alpha_{1} \alpha_{2}} \cong W_{\alpha_{1} \alpha_{2}}$. In the following, we will not distinguish $Z_{\alpha_{1} \alpha_{2}}$ from $W_{\alpha_{1} \alpha_{2}}$ unless we mention otherwise.

We now give the tangent-obstruction complex of $\mathfrak{F}_{\alpha_{1} \alpha_{2}}$. Let $S$ be any affine scheme and let $\xi \in \mathfrak{F}_{\alpha_{1} \alpha_{2}}(S)$ be represented by $f: \mathcal{X} \rightarrow X$ with the marked divisor $D \subset \mathcal{X}$ and the distinguished nodal section $d: S \rightarrow \mathcal{X}$. Let $\mathcal{B}^{\bullet}(\xi)$ be the complex $\left[f^{*} \Omega_{X} \rightarrow \Omega_{\mathcal{X} / S}^{0}(D)\right]$ indexed at -1 and 0 , where $\Omega_{\mathcal{X} / S}^{0}$ is the quotient sheaf of $\Omega_{\mathcal{X} / S}$ by its torsion supported at the distinguished nodal section and $f^{*} \Omega_{X} \rightarrow \Omega_{\mathcal{X} / S}^{0}$ is induced by $f^{*} \Omega_{X} \rightarrow \Omega_{\mathcal{X} / S}$. Because the first order deformations of nodal curves with distinguished nodal sections are $\operatorname{Ext}_{\mathcal{X}}^{1}\left(\Omega_{\mathcal{X} / S}^{0}, \mathcal{O}_{\mathcal{X}}\right)$, from the description of the tangent-obstruction complex $\mathcal{T}^{\bullet} \mathfrak{F}_{\alpha, g, n}^{X}$, we see immediately that

$$
\mathcal{T}^{1} \mathfrak{F}_{\alpha_{1} \alpha_{2}}(\xi)(\mathcal{F})=\mathcal{E} x t_{\mathcal{X} / S}^{1}\left(\mathcal{B}^{\bullet}(\xi), \pi_{S}^{*} \mathcal{F}\right)
$$

where $\pi_{S}: \mathcal{X} \rightarrow S$ is the projection, and that there is an obstruction theory to deformations of $\mathfrak{F}_{\alpha_{1} \alpha_{2}}$ taking values in

$$
\mathcal{T}^{2} \mathfrak{F}_{\alpha_{1} \alpha_{2}}(\xi)(\mathcal{F})=\mathcal{E} x t_{\mathcal{X} / S}^{2}\left(\mathcal{B}^{\bullet}(\xi), \pi_{S}^{*} \mathcal{F}\right)
$$


Now let $\hat{T}$ be the formal completion of $\mathcal{X}$ along $d(S)$. It follows that we have the long exact sequence

$$
\begin{aligned}
0 \longrightarrow \mathcal{T}^{1} \mathfrak{F}_{\alpha_{1} \alpha_{2}}(\xi)(\mathcal{F}) \longrightarrow & \mathcal{T}^{1} \mathfrak{F}_{\alpha, g, n}^{X}(\xi)(\mathcal{F}) \stackrel{c}{\longrightarrow} \mathcal{E} x t_{\hat{T} / S}^{1}\left(\Omega_{\hat{T} / S}, \pi_{S}^{*} \mathcal{F}\right) \longrightarrow \\
\stackrel{b}{\longrightarrow} & \mathcal{T}^{2} \mathfrak{F}_{\alpha_{1} \alpha_{2}}(\xi)(\mathcal{F}) \longrightarrow \mathcal{T}^{2} \mathfrak{F}_{\alpha, g, n}^{X}(\xi)(\mathcal{F}) \longrightarrow 0 .
\end{aligned}
$$

Note that $\mathcal{E} x t_{\hat{T} / S}^{1}\left(\Omega_{\hat{T} / S}, \pi_{S}^{*} \mathcal{F}\right) \cong \mathcal{F}$. It follows from $[\mathrm{DM}]$ that $\mathcal{E} x t_{\hat{T} / S}^{1}\left(\Omega_{\hat{T} / S}, \mathcal{O}_{\hat{T}}\right)$ is isomorphic to the pull back of the $\mathbf{Q}$-invertible sheaf $\mathcal{O}_{\mathcal{M}_{\alpha, g, n}^{X}}\left(L_{\alpha_{1} \alpha_{2}}\right)$ and the homomorphism $b$ is induced by the differential of the defining equation $f_{\alpha_{1} \alpha_{2}}$. Now let $S \rightarrow Y_{0} \rightarrow Y$ be the triple described in Definition 1.2 and let $\xi_{0}$ be a family in $\mathfrak{F}_{\alpha_{1} \alpha_{2}}\left(Y_{0}\right)$ extending $\xi$. Let $o$ (resp. $\left.\tilde{o}\right)$ be the obstruction class to extending $\xi_{0}$ to $\mathfrak{F}_{\alpha_{1} \alpha_{2}}(Y)$ (resp. to $\mathfrak{F}_{\alpha, g, n}^{X}(Y)$ ). A straightforward analysis of the definitions of $o$ and $\tilde{o}$ shows that $\tilde{o}=c(o)$. Next, assume that $\tilde{o}=0$. Namely $\xi_{0}$ extends to a family $\tilde{f}: \tilde{\mathcal{X}} \rightarrow X$ over $Y$. Let $h \in \operatorname{Ext}_{\hat{T}}^{1}\left(\Omega_{\hat{T} / S}, \pi_{S}^{*} \mathcal{I}_{Y_{0} \subset Y}\right)$ be the section associated to the family $\tilde{\mathcal{X}} \rightarrow Y$. Then it is direct to check that $o$ is the image of $h$ under $b$ (see $[\mathrm{DM}])$. This proves that the obstruction theory of $\mathfrak{F}_{\alpha_{1} \alpha_{2}}$ and $\mathfrak{F}_{\alpha, g, n}^{X}$ are compatible with respect to the defining equation $f_{\alpha_{1} \alpha_{2}}=0$. Finally, following the construction of the complex $\mathcal{E}^{\bullet}$ in the beginning of section 4, we can find complexes $\mathcal{E}^{\bullet}$ and $\mathcal{F}^{\bullet}$ such that $\mathfrak{h}^{\bullet}\left(\mathcal{E}^{\bullet}\right)=\mathcal{T} \bullet \mathfrak{F}_{\alpha_{1} \alpha_{2}}$ and $\mathfrak{h}^{\bullet}\left(\mathcal{F}^{\bullet}\right)=\mathcal{T} \bullet \mathfrak{F}_{\alpha, g, n}^{X}$ which satisfy the technical condition of Proposition 3.9. Therefore, by the argument at the end of the proof of Theorem 4.2, we can apply Proposition 3.9 to conclude

$$
c_{1}\left(\left[L_{\alpha_{1} \alpha_{2}}, f_{\alpha_{1} \alpha_{2}}\right]\right)\left[\mathcal{M}_{\alpha, g, n}^{X}\right]^{\mathrm{vir}}=\left[Z_{\alpha_{1} \alpha_{2}}\right]^{\mathrm{vir}} .
$$

In the following discussions, we will abbreviate $\mathfrak{F}_{\alpha_{i}, g_{i}, n_{i}+1}^{X}$ to $\mathfrak{F}_{\alpha_{i}}$. It remains to show that $\mathcal{T}^{\bullet} \mathfrak{F}_{\alpha_{1} \alpha_{2}}$ is compatible to the tangent-obstruction complex of $\mathcal{T} \bullet\left(\mathfrak{F}_{\alpha_{1}} \times \mathfrak{F}_{\alpha_{2}}\right)$ with respect to the fiber product

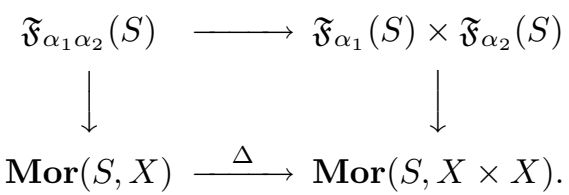

Here, given $\xi \in \mathfrak{F}_{\alpha_{1} \alpha_{2}}(S)$ represented by the map $f$ and the distinguished section $d$, the first vertical arrow will send it to $f \circ d: S \rightarrow X$. Similarly, for $\left(\xi_{1}, \xi_{2}\right) \in$ $\mathfrak{F}_{\alpha_{1}} \times \mathfrak{F}_{\alpha_{2}}(S)$ represented by the pair of maps $f_{1}$ and $f_{2}$, the second arrow will send it to $\left(e_{n_{1}+1}, e_{1}\right): S \rightarrow X \times X$, where $e_{n_{1}+1}$ is the $\left(n_{1}+1\right)$-th evaluation map of $f_{1}$ and $e_{1}$ is the first evaluation map of $f_{2}$. Let $\xi \in \mathfrak{F}_{\alpha_{1} \alpha_{2}}(S)$ be as before. By decomposing $f$ along its distinguished section $d$, we obtain a pair of families $\left\{f_{i}: D_{i} \subset \mathcal{X}_{i} \rightarrow X\right\}=\xi_{i} \in \mathfrak{F}_{\alpha_{i}}(S)$ for $i=1,2$. We let

$$
\mathcal{R}_{i}^{\bullet}(\xi)=\left[f_{i}^{*} \Omega_{X}\left(-d_{i}(S)\right) \rightarrow \Omega_{\mathcal{X}_{i} / S}\left(D_{i}-d_{i}(S)\right)\right],
$$

where $d_{1}$ is the last marked section of $D_{1} \subset \mathcal{X}_{1}$ and $d_{2}$ is the first marked section of $D_{2} \subset \mathcal{X}_{2}$. Let $\iota_{i}: \mathcal{X}_{i} \rightarrow \mathcal{X}$ be the immersion. Then we have the exact sequence

$$
0 \longrightarrow \iota_{1 *} \mathcal{R}_{1}^{\bullet}(\xi) \oplus \iota_{2 *} \mathcal{R}_{2}^{\bullet}(\xi) \longrightarrow \mathcal{B}^{\bullet}(\xi) \longrightarrow\left[f^{*} \Omega_{X} \otimes_{\mathcal{O}_{\mathcal{X}}} \mathcal{O}_{d(S)} \rightarrow 0\right] \longrightarrow 0
$$

and its induced long exact sequence

$$
\begin{gathered}
0 \longrightarrow \mathcal{E}_{t_{\mathcal{X} / S}^{1}}^{1}\left(\mathcal{B}^{\bullet}(\xi), \pi_{S}^{*} \mathcal{F}\right) \longrightarrow \oplus_{i=1}^{2} \mathcal{E} x t_{\mathcal{X} / S}^{1}\left(\iota_{i *} \mathcal{R}_{i}^{\bullet}(\xi), \pi_{S}^{*} \mathcal{F}\right) \longrightarrow \\
\stackrel{h_{1}}{\longrightarrow} \mathcal{E} x t_{\mathcal{X} / S}^{1}\left(f^{*} \Omega_{X} \otimes_{\mathcal{O}_{\mathcal{X}}} \mathcal{O}_{d(S)}, \pi_{S}^{*} \mathcal{F}\right) \stackrel{h_{2}}{\longrightarrow} \mathcal{E}_{t} t_{\mathcal{X} / S}^{2}\left(\mathcal{B}^{\bullet}(\xi), \pi_{S}^{*} \mathcal{F}\right) \longrightarrow \\
\longrightarrow \oplus_{i=1}^{2} \mathcal{E} x t_{\mathcal{X} / S}^{2}\left(\iota_{i *} \mathcal{R}_{i}^{\bullet}(\xi), \pi_{S}^{*} \mathcal{F}\right) \longrightarrow 0
\end{gathered}
$$


It is direct to check that there are canonical isomorphisms

$$
\mathcal{E}_{x} t_{\mathcal{X} / S}^{j}\left(\iota_{i *} \mathcal{R}_{i}^{\bullet}(\xi), \pi_{S}^{*} \mathcal{F}\right) \cong \mathcal{E}_{x} t_{\mathcal{X}_{i} / S}^{j}\left(\left[f_{i}^{*} \Omega_{X} \rightarrow \Omega_{\mathcal{X}_{i} / S}\left(D_{i}\right)\right], \pi_{S}^{*} \mathcal{F}\right)
$$

and

$$
\mathcal{E}_{x} t_{\mathcal{X} / S}^{1}\left(f^{*} \Omega_{X} \otimes_{\mathcal{O}_{\mathcal{X}}} \mathcal{O}_{d(S)}, \pi_{S}^{*} \mathcal{F}\right) \cong(f \circ d)^{*} \Omega_{X}^{\vee} \otimes_{\mathcal{O}_{S}} \mathcal{F}
$$

Therefore, (5.8) is the long exact sequence of cohomologies mentioned in Definition 3.8. Now we show that the tangent-obstruction complexes of $\mathfrak{F}_{\alpha_{1} \alpha_{2}}$ and of $\mathfrak{F}_{\alpha_{1}} \times \mathfrak{F}_{\alpha_{2}}$ are compatible with respect to the defining diagram (5.7). Let $\xi \in \mathfrak{F}_{\alpha_{1} \alpha_{2}}(S)$ be as before and let $\xi_{i} \in \mathfrak{F}_{\alpha_{i}}(S)$ be represented by the family $f_{i}$. We first show that the canonical homomorphism

$\mathcal{T}^{1}\left(\mathfrak{F}_{\alpha_{1}} \times \mathfrak{F}_{\alpha_{2}}\right)\left(\xi_{1}, \xi_{2}\right)(\mathcal{F}) \longrightarrow\left(e_{n_{1}+1}, e_{1}\right)^{*} \mathcal{O}\left(T_{X \times X}\right) \otimes_{\mathcal{O}_{S}} \mathcal{F} \longrightarrow(f \circ d)^{*} \Omega_{X}^{\vee} \otimes_{\mathcal{O}_{S}} \mathcal{F}$ is the homomorphism $h_{1}$ in the exact sequence (5.8). Let $S$ be affine, let $\mathcal{F} \in \mathfrak{M o d} \mathfrak{d}_{S}$ and let $v_{1} \in \mathcal{T}^{1} \mathfrak{F}_{\alpha_{1}}\left(\xi_{1}\right)(\mathcal{F})$ be represented by a flat extension $\tilde{f}_{1}$ of $f_{1}$. Let

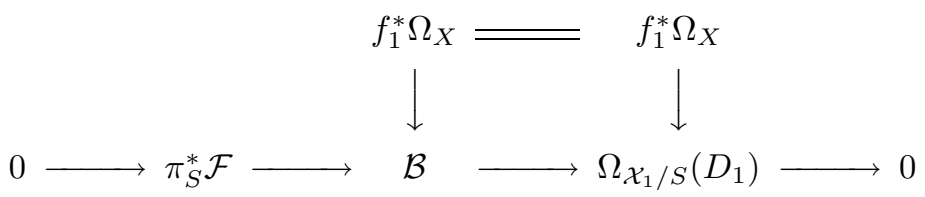

be the associated diagram. We first set $\mathcal{B}^{\prime}$ to be the sheaf defined by the pushforward diagram

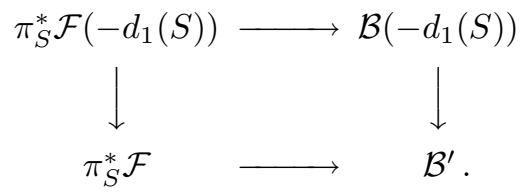

Then $f_{1}^{*} \Omega_{X}\left(-d_{1}(S)\right) \rightarrow \mathcal{B}^{\prime}$ lifts to $f_{1}^{*} \Omega_{X} \rightarrow \mathcal{B}^{\prime}$. Let $\mathcal{B}_{1}^{\bullet}\left(\xi_{1}\right)=\left[f_{1}^{*} \Omega_{X} \rightarrow \Omega_{\mathcal{X}_{1} / S}(D)\right]$ and let $p\left(\xi_{1}\right)$ be the homomorphism

$$
\mathcal{T}^{1} \mathfrak{F}_{\alpha_{1}}\left(\xi_{1}\right)(\mathcal{F})=\mathcal{E} x t_{\mathcal{X}_{1} / S}^{1}\left(\mathcal{B}_{1}^{\bullet}\left(\xi_{1}\right), \pi_{S}^{*} \mathcal{F}\right) \stackrel{p\left(\xi_{1}\right)}{\longrightarrow} e_{n_{1}+1}^{*} \Omega_{X}^{\vee} \otimes_{\mathcal{O}_{S}} \mathcal{F}
$$

that sends $v_{1}$ to the composite

$$
e_{n_{1}+1}^{*} \Omega_{X} \longrightarrow e_{n_{1}+1}^{*} \mathcal{B}^{\prime} \longrightarrow \operatorname{coker}\left\{\mathcal{B}\left(-d_{1}(S)\right) \rightarrow \mathcal{B}^{\prime}\right\} \equiv \mathcal{F}
$$

One checks directly that $p\left(\xi_{1}\right)$ assigns the flat extension $\tilde{f}_{1}$ to the tangent direction of $\tilde{f} \circ \tilde{e}_{n_{1}+1}$, where $\tilde{e}_{n_{1}+1}$ is the $\left(n_{1}+1\right)$-th marked section of $\tilde{f}$. Similarly, we let $p\left(\xi_{2}\right)$ be

$$
p\left(\xi_{2}\right): \mathcal{T}^{1} \mathfrak{F}_{\alpha_{2}}\left(\xi_{2}\right)(\mathcal{F})=\mathcal{E} x t_{\mathcal{X}_{2} / S}^{1}\left(\mathcal{B}_{2}^{\bullet}\left(\xi_{2}\right), \pi_{S}^{*} \mathcal{F}\right) \longrightarrow e_{1}^{*} \Omega_{X}^{\vee} \otimes_{\mathcal{O}_{S}} \mathcal{F}
$$

that is defined with $f_{1}$, etc. replaced by $f_{2}$, etc. respectively. Then $p\left(\xi_{1}\right)-$ $p\left(\xi_{2}\right)$ is the homomorphism $h_{1}$ in the exact sequence (5.8). Therefore, the induced homomorphism $\left(e_{n_{1}+1}, e_{1}\right)$ on the tangent spaces

$$
\mathcal{T}^{1}\left(\mathfrak{F}_{\alpha_{1}} \times \mathfrak{F}_{\alpha_{2}}\right)\left(\xi_{1}, \xi_{2}\right)(\mathcal{F}) \longrightarrow\left(e_{n_{1}+1}, e_{1}\right)^{*} \Omega_{X \times X}^{\vee} \otimes_{\mathcal{O}_{S}} \mathcal{F}
$$

coincides with $\left(p\left(\xi_{1}\right), p\left(\xi_{2}\right)\right)$, and consequently, its composite with

$$
\left(e_{n_{1}+1}, e_{1}\right)^{*} \Omega_{X \times X}^{\vee} \otimes_{\mathcal{O}_{S}} \mathcal{F} \longrightarrow\left(e_{n_{1}+1}, e_{1}\right)^{*} \mathcal{N}_{\Delta(X) / X \times X} \otimes_{\mathcal{O}_{S}} \mathcal{F}
$$

is $p\left(\xi_{1}\right)-q\left(\xi_{2}\right)$, after identifying the normal bundle $N_{\Delta(X) / X \times X}$ with $T_{X}$. Similarly, it is direct to check that the obstruction classes to extending a given family to families in $\mathfrak{F}_{\alpha_{1} \alpha_{2}}$ and to families in $\mathfrak{F}_{\alpha_{1}} \times \mathfrak{F}_{\alpha_{2}}$ are compatible in the sense of Definition 
3.8. Finally, similar to the case studied before, we can construct complexes of locally free sheaves whose sheaf cohomologies are the tangent-obstruction complexes of $\mathfrak{F}_{\alpha_{1}} \times \mathfrak{F}_{\alpha_{2}}$ required by Proposition 3.9. Therefore, by applying Proposition 3.9, we have

$$
\Delta^{!}\left(\left[\mathcal{M}_{\alpha_{1}, g_{1}, n_{1}+1}^{X} \times \mathcal{M}_{\alpha_{2}, g_{2}, n_{2}+1}^{X}\right]^{\mathrm{vir}}\right)=\left[Z_{\alpha_{1} \alpha_{2}}\right]^{\mathrm{vir}} .
$$

Finally, we choose a sufficiently large $l$ such that $L_{\alpha_{1} \alpha_{2}}^{\otimes l}$ and $L_{n}^{\otimes l}$ are conventional line bundles. Then

$$
\begin{aligned}
& l \cdot\left(\tau_{g_{\bullet} n \bullet}\right)^{!}\left[\mathcal{M}_{\alpha, g, n}^{X}\right]^{\mathrm{vir}}=c_{1}\left(\left(\pi_{n}^{\alpha}\right)^{*} L_{n}^{\otimes l}\right)\left[\mathcal{M}_{\alpha, g, n}^{X}\right]^{\mathrm{vir}} \\
= & \sum_{\alpha_{1}+\alpha_{2}=\alpha} c_{1}\left(L_{\alpha_{1} \alpha_{2}}^{\otimes l}\right)\left[\mathcal{M}_{\alpha, g, n}^{X}\right]^{\mathrm{vir}}=\sum_{\alpha_{1}+\alpha_{2}=\alpha} l \cdot c_{1}\left(\left[L_{\alpha_{1} \alpha_{2}}, f_{\alpha_{1} \alpha_{2}}\right]\right)\left[\mathcal{M}_{\alpha, g, n}^{X}\right]^{\mathrm{vir}} .
\end{aligned}
$$

By (5.6), the terms in the last summation are $l \cdot\left[Z_{\alpha_{1} \alpha_{2}}\right]^{\text {vir }}$. Combined with (5.9), we obtain

$$
\left(\tau_{g_{\bullet} n \bullet}\right)^{!}\left[\mathcal{M}_{\alpha, g, n}^{X}\right]^{\mathrm{vir}}=\sum_{\alpha_{1}+\alpha_{2}=\alpha} \Delta^{!}\left(\left[\prod_{i=1}^{2} \mathcal{M}_{\alpha_{i}, g_{i}, n_{i}+1}^{X}\right]^{\mathrm{vir}}\right) .
$$

This proves the first composition law.

In the end, we will indicate the necessary change needed to prove the second composition law. Let $Z_{1}$ and $Z_{2}$ be the $\mathbf{Q}$-schemes and $\Phi$ be the morphism defined in the statement of the theorem. For convenience, we will consider $Z_{1} / \mathbf{Z}_{2}$ and $Z_{2} / \mathbf{Z}_{2}$, where $\mathbf{Z}_{2}$ acts on $Z_{1}$ and $Z_{2}$ by interchanging the last two marked points of the curves in $\mathcal{M}_{\alpha, g-1, n+2}^{X}$ and $\mathcal{M}_{g-1, n+2}$. Let $Z_{i}^{\prime}=Z_{i} / \mathbf{Z}_{2}$. Clearly, $\Phi$ factors through $\Psi: Z_{2}^{\prime} \rightarrow Z_{1}^{\prime}$. $\Psi$ is a local embedding in the sense that it is finite and unramified. Let $\left(L_{n}, f_{n}\right)$ be the $\mathbf{Q}$-line bundle and its section on $\mathcal{M}_{g, n}$ such that $f_{n}=0$ defines the image $\mathbf{Q}$-scheme $\mathcal{M}_{g-1, n+2} \rightarrow \mathcal{M}_{g, n}$. We pick a $w \in Z_{2}$ and let $\left\{z_{1}, \cdots, z_{k}\right\}=\Psi^{-1}(w)$. Now let $W \rightarrow \mathcal{M}_{\alpha, g, n}^{X}$ be a chart of $\mathcal{M}_{\alpha, g, n}^{X}$ containing $w$ with the tautological family $\xi$. We let $U_{i} \rightarrow Z_{1}^{\prime}$ be the charts of $Z_{1}^{\prime}$ containing $z_{i}$ with the tautological family $\eta_{i}$. Recall that each family $\eta_{i}$ has a distinguished section of nodal points. Without loss of generality, we can assume that there are morphisms $\varphi_{i}: U_{i} \rightarrow W$ such that $\varphi_{i}^{*}(\xi)=\eta_{i}$. Now by using the technique of adding extra sections, we can find an étale covering $\tilde{W} \rightarrow W, k$ sections $g_{1}, \cdots, g_{k} \in \mathcal{O}_{\tilde{W}}$ and étale covering $\tilde{U}_{i} \rightarrow U_{i}$ of which the following holds. First, after fixing a trivialization of $L_{n}$ over a chart $V$ of $\mathcal{M}_{g, n}$, the product $\prod^{k} g_{i}$ is the pull back of $f_{n}$ under the obvious map $\tilde{W} \rightarrow V$; second, there are morphisms $\tilde{\varphi}_{i}: \tilde{V}_{i} \rightarrow \tilde{W}$ making the diagram

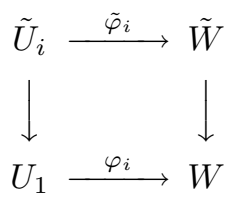

commutative such that $\tilde{\varphi}_{i}$ are embeddings and the image schemes $\tilde{\varphi}_{i}\left(\tilde{U}_{i}\right)=\left\{g_{i}=\right.$ $0\}$. Using the distinguished section of nodal points in the family $\eta_{i}$, one can construct a $\mathbf{Q}$-invertible sheaf $\mathcal{L}$ on $Z_{1}^{\prime}$ such that over the chart $\tilde{U}_{i}$, it is the locally free sheaf defined similarly to the far right term in (5.2). For convenience, let us assume that $\left[\mathcal{M}_{\alpha, g, n}^{X}\right]^{\text {vir }}$ is a cycle $R$ supported on an equidimensional scheme with 
multiplicity. Now let $Y_{i}=\left\{g_{i}=0\right\} \subset \tilde{W}$ and let $R_{\tilde{W}}$ be the pull back of $R$ under $\tilde{W} \rightarrow \mathcal{M}_{\alpha, g, n}^{X}$. Consider the normal cone cycle

$$
\left[C_{R_{\tilde{W}} \times \tilde{W} Y_{i} / R_{\tilde{W}}}\right]
$$

Using the isomorphism $\tilde{\varphi}_{i}: \tilde{U}_{i} \rightarrow Y_{i}$, one can pull back the cycles $\left[C_{R_{\tilde{W}} \times \tilde{W} Y_{i} / R_{\tilde{W}}}\right]$ and patch them together to form a global cycle in the total space of

$$
\operatorname{Vect}_{Z_{1}^{\prime}}(\mathcal{L}) \times_{\mathcal{M}_{\alpha, g, n}^{X}} R
$$

We denote this cycle by $\mathbf{D}$, and the zero section of $\operatorname{Vect}_{Z_{1}^{\prime}}(\mathcal{L}) \times_{\mathcal{M}_{\alpha, g, n}^{X}} R$ by $\zeta$. Then by studying the tangent-obstruction complex of $Z_{1}^{\prime}$ induced by the defining equation $g_{i}=0$ and that of $Z_{1}^{\prime}$ induced by the defining square of $Z_{1}$ in the statement of the theorem, we conclude that

$$
2 \Psi_{*}\left(\zeta^{*}[\mathbf{D}]\right)=\iota_{*} \Phi_{*}\left(\Delta^{!}\left[\mathcal{M}_{\alpha, g-1, n+2}^{X}\right]^{\mathrm{vir}}\right),
$$

where $\iota: Z_{2} \rightarrow Z_{2}^{\prime}$ is the projection. However, it is clear that

$$
\iota_{*}\left(\tau_{g-1, n+2}\right)^{!}\left[\mathcal{M}_{\alpha, g, n}^{X}\right]^{\mathrm{vir}}=2 c_{1}\left(\left[\left(\tau_{n}^{\alpha}\right)^{*}\left(L_{n}\right),\left(\tau_{n}^{\alpha}\right)^{*}\left(f_{n}\right)\right]\right)(R) .
$$

Therefore, the second composition law will follow from

$$
c_{1}\left(\left[\left(\tau_{n}^{\alpha}\right)^{*}\left(L_{n}\right),\left(\tau_{n}^{\alpha}\right)^{*}\left(f_{n}\right)\right]\right)(R)=\Psi_{*}\left(\zeta^{*}[\mathbf{D}]\right) .
$$

But this can can be checked directly. This proves the second composition law.

\section{REFERENCES}

[Al] V. Alexeev, Moduli spaces $M_{g, n}(W)$ for surfaces, preprint.

[At] I. V. Artamkin, On deformation of sheaves, Math. USSR Izv. 32 (no. 3) (1989), 663-668. MR 89g:14004

[Be] A. Beauville, Quantum cohomology of complete intersections, preprint.

[Bh] K. Behrend, The Gromov-Witten invariants, Invent. Math. 127 no. 3 (1997), 601-617. CMP 97:07

[BF] K. Behrend and B. Fantechi, The intrinsic normal cone, Invent. Math. 128 no. 1 (1997), 45-88. CMP 97:09

[Ber] A. Bertram, Quantum Schubert Calculus, Advance in Math. 128 (1997), 289-305. CMP 97:14

[BDW] A. Bertram, G. Daskalopoulos and R. Wentworth, Gromov invariants for holomorphic maps from Riemann surfaces to Grassmannians, J. Amer. Math. Soc. 9 no. 2 (1996), 529-571. MR 96f: 14066

[Ci] I. Ciocan-Fontanine, Quantum cohomology of flag varieties, Internat. Math. Res. Notices $6(1995),, 263-277$. MR 96h:14071

$[\mathrm{CM}]$ B. Crauder and R. Mirander, Quantum cohomology of rational surfaces, The Moduli Space of Curves (R. Dijkgraaf, C. Faber, G. Van der Geer, eds.), Progress in Mathematics 129, 1995, 33-80. MR 96g:14002

[DM] P. Deligne and D. Mumford, The irreducibility of the space of curves of given genus, Publ. I.H.E.S. 45 (1969), 101-145. MR 41:6850

[Do] S. Donaldson, Polynomial invariants for smooth four manifolds, Topology 29 (1990), 257-315. MR 92a:57035

[ES] G. Ellingsrud and S. Stromme, The number of twisted cubic curves on the generic quintic threefold, Math. Scand. 76 no. 1 (1995), 5-34. MR 96g:14045

[FO] K. Fukaya and K. Ono, Arnold conjecture and Gromov-Witten invariants, preprint (1996).

[Fu] W. Fulton, Intersection theory, Ergebnisse der Math. und ihrer Grenzgebiete 3. Folge Band 2, 1984. MR 85k:14004

[FP] W. Fulton and R. Pandharipande, Notes on stable maps and quantum cohomology, to appear in the Proceeding of AMS conference in algebraic geometry, Santa Cruz.

[GH] P. Griffiths and J. Harris, Principles of algebraic geometry, John Wiley \& Sons, New York, 1978. MR 80b:14001 
[Kz1] S. Katz, Lectures at 1993 enumerative geometry conference in Dyrkolbotn, Norway, private communication.

[Kz2] S. Katz, Gromov-Witten Invariants via Algebraic Geometry, preprint.

[Ka] Y. Kawamata, Unobstructed deformations - a remark on a paper of Z. Ran, J. Alg. Geom. 1 (1992), 183-190. MR 93e:14016

[Ko1] M. Kontsevich, Intersection theory on the moduli space of curves and the matrix Airy function, Comm. Math. Phys. 147 (1992). MR 93e:32027

[Ko2] M. Kontsevich, Enumeration of rational curves via torus actions, The Moduli Space of Curves (R. Dijkgraaf, C. Faber, G. van der Geer, eds.), Progress in Mathematics vol. 129, Birkhauser 1995, 335-368. MR 97d:14077

[KM] M. Kontsevich and Y. Manin, GW classes, Quantum cohomology and enumerative geometry, Comm. Math. Phys. 164 (1994), 525-562. MR 95i:14049

[Kn] F. Knudsen, The projectivity of the moduli space of stable curves. II. The stacks $M_{g, n}$, Math. Scand. 52 (1983), no.2, 161-199. MR 85d:14038a

[La] O. A. Laudal, Formal Moduli of Algebraic Structures, Lecture Notes in Mathematics, Vol. 754, Berlin Heidelberg New York, Springer 1979. MR 82h:14009

[Li] J. Li, Algebraic geometric interpretation of Donaldson's polynomial invariants, J. Diff. Geom. 37 (1993), 417-466. MR 93m:14007

[LT] J. Li and G. Tian, Quantum cohomology of homogeneous manifolds, to appear in J. Alg. Geom.

[LT2] J. Li and G. Tian, Virtual moduli cycles and Gromov-Witten invariants of general symplectic manifolds, preprint (1996).

[Ma] H. Matsumura, Commutative ring theory, Cambridge Studies in Advanced Mathematics, 8. Cambridge University Press, Cambridge-New York, 1989. MR 90i:13001

[Mo] J. Morgan, Comparison of the Donaldson polynomial invariants with their algebrogeometric analogues, Topology 32 (no 3) (1993), 449-488. MR 94m:57066

$[\mathrm{Mu}]$ D. Mumford, Towards an enumerative geometry of the moduli space of curves, Arithmetic and Geometry II, Progress in Mathematics 36 (1983), 271-326. MR 85j:14046

[Ra] Z. Ran, Deformations of maps, Algebraic Curves and Projective Geometry (E. Ballico, C. Ciliberto, eds.), Lecture Notes in Math., vol. 1389. MR 91f:32021

[Ru] Y. Ruan, Topological Sigma model and Donaldson type invariants in Gromov theory, Duke Math. J. 83 no. 2 (1996), 461-500. MR 97d:58042

[RQ] Y. Ruan and Z-B. Qin, Quantum cohomology of projective bundles over $\mathbf{P}^{n}$, to appear in Trans. Amer. Math. Soc. CMP 97:05

[RT1] Y. Ruan and G. Tian, A mathematical theory of quantum cohomology, J. Diff. Geom. 42 no. 2 (1995), 259-367. MR 96m:58033

[RT2] Y. Ruan and G. Tian, Higher genus symplectic invariants and sigma model coupled with gravity, Turkish J. Math. 20 no. 1 (1996), 75-83. MR 97d:58029

[Si] B. Siebert, Gromov-Witten invariants for general symplectic manifolds, preprint (1996).

[Ti] G. Tian, Quantum cohomology and its associativity, To appear in Proc. of 1st Current Developments in Math., Cambridge (1995).

[Vi] A. Vistoli, Intersection theory on algebraic stacks and on their moduli spaces, Invent. Math. 97 (1989), 613-670. MR 90k:14004

[Vo] C. Voisin, A mathematical proof of Aspinwall-Morrison formula, Compositio Math. 104 no. 2 (1996), 135-151. CMP 97:04

[W1] E. Witten, Topological sigma models, Comm. Math. Phys. 118 (1988). MR 90b:81080

[W2] E. Witten, Two dimensional gravity and intersection theory on moduli space, Surveys in Diff. Geom. 1 (1991), 243-310. MR 93e:32028

Department of Mathematics, Stanford University, Stanford, California 94305

E-mail address: jli@gauss.stanford.edu

Department of Mathematics, Massachusetts Institute of Technology, Cambridge, MASSACHUSETTS 02139

E-mail address: tian@math.mit.edu 